\title{
HILBERT'S FIFTH PROBLEM FOR LOCAL GROUPS
}

\author{
ISAAC GOLDBRING
}

\begin{abstract}
We solve Hilbert's fifth problem for local groups: every locally euclidean local group is locally isomorphic to a Lie group. Jacoby claimed a proof of this in 1957, but this proof is seriously flawed. We use methods from nonstandard analysis and model our solution after a treatment of Hilbert's fifth problem for global groups by Hirschfeld.
\end{abstract}

\section{INTRODUCTION}

The most common version of Hilbert's fifth problem asks whether every locally euclidean topological group is a Lie group. More precisely, if $G$ is a locally euclidean topological group, does there always exist a $C^{\omega}$ structure on $G$ such that the group operations become $C^{\omega}$ ? In 1952, Gleason, Montgomery, and Zippin answered this question in the affirmative; see [4] and [13]. " $C C^{\omega}$ " means "real analytic," and by a $C^{\omega}$ structure on a topological space $X$ we mean a real analytic manifold, everywhere of the same finite dimension, whose underlying topological space is $X$.)

However, Lie groups, as first conceived by Sophus Lie, were not actually groups but rather "local groups" where one could only multiply elements that were sufficiently near the identity. (A precise definition of "local group" is given in the next section.) It is thus natural to pose a local version of Hilbert's fifth problem (henceforth referred to as the Local H5): Is every locally euclidean local group locally isomorphic to a Lie group? In [8], Jacoby claims to have solved the Local H5 affirmatively. However, as pointed out by Plaut in [16], Jacoby fails to recognize a subtlety in the way the associative law holds in local groups, as we will now explain.

In our local groups, if the products $x y, y z, x(y z)$, and $(x y) z$ are all defined, then $x(y z)=(x y) z$. This condition is called "local associativity" by Olver in [14]. A much stronger condition for a local group to satisfy is "global associativity" in which, given any finite sequence of elements from the local group and two different ways of introducing parentheses in the sequence, if both products thus formed exist, then these two products are in fact equal. According to [14, "Jacoby's Theorem 8, which is stated without proof, essentially makes the false claim that local associativity implies global associativity." Contrary to this "Theorem 8", 14 has examples of connected local Lie groups that are not globally associative. Since, according to [14], 
"the rest of Jacoby's paper relies heavily on his incorrect Theorem 8", this error invalidates his proof. Olver also indicates that one could probably rework Jacoby's paper to extract the truth of the Local H5. He suggests that this would be a worthwhile endeavor, as, for example, the solution of Hilbert's fifth problem for cancellative semigroups on manifolds heavily relies on the truth of the Local H5; see [1] and [7].

Rather than reworking Jacoby's paper, we have decided to mimic the nonstandard treatment of Hilbert's fifth problem given by Hirschfeld [6]. Whereas nonstandard methods simplified the solution of Hilbert's fifth problem, such methods are even more natural in dealing with the Local H5. The nonstandard proof of Hilbert's fifth problem involves "infinitesimals"elements in the nonstandard extension of the group infinitely close to the identity; therefore, much of [6] goes through in the local setting, since the infinitesimals form an actual group. These infinitesimals are used to generate local one-parameter subgroups, as in [17] and [6].

While our solution of the Local H5 is along the lines of the proof given by Hirschfeld, many of the arguments have grown substantially in length due to the care needed in working with local groups. We assume familiarity with elementary nonstandard analysis; otherwise, consult [2] or [5]. We should also mention that McGaffey [11] has a completely different nonstandard approach to Hilbert's fifth problem for local groups, using the connection between local Lie groups and finite-dimensional real Lie algebras to reduce to a problem of approximating locally euclidean local groups in a certain metric by local Lie groups. This approach has not yet led to a solution.

I would like to thank Lou van den Dries for many helpful suggestions.

\section{Preliminaries}

In this section, we define the notion of local group and state precisely the Local H5 (in two forms). We also introduce the fundamental objects in the entire story, the local 1-parameter subgroups of $G$. After defining the local versions of familiar constructions in group theory, e.g. morphisms between local groups, sublocal groups, and local quotient groups, we prove some elementary properties of local groups that are used heavily throughout the paper. We end this section by fixing some notation concerning the nonstandard setting.

Throughout, $m$ and $n$ range over $\mathbb{N}:=\{0,1,2, \ldots\}$. 


\section{Statement of the Local H5}

Definition 2.1. A local group is a tuple $(G, 1, \iota, p)$ where $G$ is a hausdorff topological space with a distinguished element $1 \in G$, and $\iota: \Lambda \rightarrow G$ (the inversion map) and $p: \Omega \rightarrow G$ (the product map) are continuous functions with open $\Lambda \subseteq G$ and open $\Omega \subseteq G \times G$, such that $1 \in \Lambda,\{1\} \times G \subseteq \Omega$, $G \times\{1\} \subseteq \Omega$, and for all $x, y, z \in G$ :

(1) $p(1, x)=p(x, 1)=x$;

(2) if $x \in \Lambda$, then $(x, \iota(x)) \in \Omega,(\iota(x), x) \in \Omega$ and

$$
p(x, \iota(x))=p(\iota(x), x)=1 ;
$$

(3) if $(x, y),(y, z) \in \Omega$ and $(p(x, y), z),(x, p(y, z)) \in \Omega$, then

$$
p(p(x, y), z)=p(x, p(y, z)) .
$$

In the rest of this paper $(G, 1, \iota, p)$ is a local group; for simplicity we denote it just by $G$. For the sake of readability, we write $x^{-1}$ instead of $\iota(x)$, and $x y$ or $x \cdot y$ instead of $p(x, y)$. Note that $\iota(1)=1$ and if $x, y \in \Lambda,(x, y) \in \Omega$, and $x y=1$, then $y=x^{-1}$ and $x=y^{-1}$.

\section{Definition 2.2.}

(1) Let $U$ be an open neighborhood of 1 in $G$. Then the restriction of $G$ to $U$ is the local group $G \mid U:=\left(U, 1, \iota\left|\Lambda_{U}, p\right| \Omega_{U}\right)$, where

$$
\Lambda_{U}:=\Lambda \cap U \cap \iota^{-1}(U) \text { and } \Omega_{U}:=\Omega \cap(U \times U) \cap p^{-1}(U) .
$$

(2) $G$ is locally euclidean if there is an open neighborhood of 1 homeomorphic to an open subset of $\mathbb{R}^{n}$ for some $n$.

(3) $G$ is a local Lie group if $G$ admits a $C^{\omega}$ structure such that the maps $\iota$ and $p$ are $C^{\omega}$.

(4) $G$ is globalizable if there is a topological group $H$ and an open neighborhood $U$ of $1_{H}$ in $H$ such that $G=H \mid U$.

By a restriction of $G$ we mean a local group $G \mid U$ where $U$ is an open neighborhood of 1 in $G$.

Local H5-First Form: If $G$ is a locally euclidean local group, then some restriction of $G$ is a local Lie group.

Local H5-Second Form: If $G$ is a locally euclidean local group, then some restriction of $G$ is globalizable.

The equivalence of the two forms of the Local H5 is seen as follows. It is known that if $G$ is a local Lie group, then some restriction of $G$ is equal to some restriction of a Lie group. Thus the first form implies the second form. 
Conversely, by the Montgomery-Zippin-Gleason solution to the original H5, if $G \mid U$ is globalizable, the global group containing $G \mid U$ will be locally euclidean and there will be a $C^{\omega}$ structure on it making it a Lie group. Then $G \mid U$ will be a local Lie group.

Definition 2.3. A subgroup of $G$ is a subset $H \subseteq G$ such that $1 \in H$, $H \subseteq \Lambda, H \times H \subseteq \Omega$ and for all $x, y \in H, x^{-1} \in H$ and $x y \in H$.

Note that then $H$ is an actual group with product map $p \mid H \times H$. We will say $G$ has no small subgroups, abbreviated $G$ is NSS, if there is a neighborhood $\mathcal{U}$ of 1 such that there are no subgroups $H$ of $G$ with $H \neq\{1\}$ and $H \subseteq \mathcal{U}$. In analogy with the solution to Hilbert's fifth problem in the global case, we will first show that every locally compact NSS local group has a restriction which is a local Lie group and then we will prove that every locally euclidean local group is NSS.

\section{The Sets $\mathcal{U}_{n}$}

As noted in the introduction, one obstacle in the local theory is the potential lack of generalized associativity. The next definition captures the informal idea of being able to unambiguously multiply a finite sequence of elements.

Definition 2.4. Let $a_{1}, \ldots, a_{n}, b \in G$ with $n \geq 1$. We define the notion $\left(a_{1}, \ldots, a_{n}\right)$ represents $b$, denoted $\left(a_{1}, \ldots, a_{n}\right) \rightarrow b$, by induction on $n$ as follows:

- $\left(a_{1}\right) \rightarrow b$ iff $a_{1}=b$;

- $\left(a_{1}, \ldots, a_{n+1}\right) \rightarrow b$ iff for every $i \in\{1, \ldots, n\}$, there exists $b_{i}^{\prime}, b_{i}^{\prime \prime} \in G$ such that $\left(a_{1}, \ldots, a_{i}\right) \rightarrow b_{i}^{\prime},\left(a_{i+1}, \ldots, a_{n+1}\right) \rightarrow b_{i}^{\prime \prime},\left(b_{i}^{\prime}, b_{i}^{\prime \prime}\right) \in \Omega$ and $b_{i}^{\prime} \cdot b_{i}^{\prime \prime}=b$.

By convention, we say that $\left(a_{1} \ldots a_{n}\right)$ represents 1 when $n=0$.

We will say $a_{1} \cdots a_{n}$ is defined if there is $b \in G$ such that $\left(a_{1}, \ldots, a_{n}\right)$ represents $b$; in this case we will write $a_{1} \cdots a_{n}$ for this (necessarily unique) $b$. If $a_{1} \cdots a_{n}$ is defined, then, informally speaking, all possible $n$-fold products are defined and equal. If $a_{1} \cdots a_{n}$ is defined and $a_{i}=a$ for all $i \in\{1, \ldots, n\}$, we say that $a^{n}$ is defined and denote the unique product by $a^{n}$. In particular, $a^{0}$ is always defined and equals 1 .

From now on, put $A^{\times n}:=\underbrace{A \times \cdots \times A}_{n \text { times }}$. Also, call $W \subseteq G$ symmetric if $W \subseteq \Lambda$ and $W=\iota(W)$. Note that $U \cap \iota^{-1}(U)$ is a symmetric open neighborhood of 1 in $G$ for every open neighborhood $U$ of 1 contained in $\Lambda$. 
We now prove a lemma which says that we can multiply any number of elements unambiguously provided that the elements are sufficiently close to the identity.

Lemma 2.5. There are open symmetric neighborhoods $\mathcal{U}_{n}$ of 1 for $n>0$ such that $\mathcal{U}_{n+1} \subseteq \mathcal{U}_{n}$ and for all $\left(a_{1}, \ldots, a_{n}\right) \in \mathcal{U}_{n}^{\times n}, a_{1} \cdots a_{n}$ is defined.

Proof. We let $\mathcal{U}_{1}$ be any open symmetric neighborhood of 1 and choose $\mathcal{U}_{2}$ to be a symmetric open neighborhood of 1 such that $\mathcal{U}_{2} \subseteq \mathcal{U}_{1}$ and $\mathcal{U}_{2} \times \mathcal{U}_{2} \subseteq \Omega$.

Assume inductively that $n \geq 2$ and that for $m=1, \ldots, n$,

- $\mathcal{U}_{m}$ is a symmetric open neighborhood of 1 ;

- $\mathcal{U}_{m+1} \subseteq \mathcal{U}_{m}$ if $m<n$

- for all $\left(a_{1}, \ldots, a_{m}\right) \in \mathcal{U}_{m}^{\times m}, a_{1} \cdots a_{m}$ is defined;

- the map $\varphi_{m}: \mathcal{U}_{m}^{\times m} \rightarrow G$ defined by $\varphi_{m}\left(a_{1}, \ldots, a_{m}\right)=a_{1} \cdots a_{m}$ is continuous.

Let $\mathcal{U}_{n+1}$ be a symmetric open neighborhood of 1 such that $\mathcal{U}_{n+1} \subseteq \mathcal{U}_{n}$ and $\mathcal{U}_{n+1}^{\times n} \subseteq \varphi_{n}^{-1}\left(\mathcal{U}_{2}\right)$. We show that this choice of $\mathcal{U}_{n+1}$ works. Let $\left(a_{1}, \ldots, a_{n+1}\right) \in \mathcal{U}_{n+1}^{\times(n+1)}$. We must show that $\left(a_{1}, \ldots, a_{n+1}\right)$ represents $a_{1} \cdot\left(a_{2} \cdots a_{n+1}\right)$. However, this is immediate by the inductive assumptions, using (A) several times.

From now on, the sets $\mathcal{U}_{n}$ will be as in the previous lemma. Also, if $A \subseteq \mathcal{U}_{n}$, put $A^{n}:=\left\{a_{1} \cdots a_{n} \mid\left(a_{1}, \ldots, a_{n}\right) \in A^{\times n}\right\}$.

\section{Local 1-parameter subgroups}

Definition 2.6. A local 1-parameter subgroup of $G$, henceforth abbreviated local 1-ps of $G$, is a continuous map $X:(-r, r) \rightarrow G$, for some $r \in(0, \infty]$, such that

(1) image $(X) \subseteq \Lambda$, and

(2) if $r_{1}, r_{2}, r_{1}+r_{2} \in(-r, r)$, then $\left(X\left(r_{1}\right), X\left(r_{2}\right)\right) \in \Omega$ and

$$
X\left(r_{1}+r_{2}\right)=X\left(r_{1}\right) \cdot X\left(r_{2}\right) \text {. }
$$

Suppose $X:(-r, r) \rightarrow G$ is a local 1-ps of $G$ and $s \in(-r, r)$. It is easy to verify, by induction on $n$, that if $n s \in(-r, r)$, then $X(s)^{n}$ is defined and $X(n s)=X(s)^{n}$.

We now define the local analog of the space which played a key role in the solution of Hilbert's fifth problem in the global setting. 
Definition 2.7. Let $X, Y$ be local 1-parameter subgroups of $G$. We say that $X$ is equivalent to $Y$ if there is $r \in \mathbb{R}^{>0}$ such that

$$
r \in \operatorname{domain}(X) \cap \operatorname{domain}(Y) \text { and } X|(-r, r)=Y|(-r, r) .
$$

We let $[X]$ denote the equivalence class of $X$ with respect to this equivalence relation. We also let $L(G):=\{[X] \mid X$ is a local 1-ps of $G\}$.

In other words, $L(G)$ is the set of germs at 0 of local 1-parameter subgroups of $G$. We often write $\mathbb{X}$ for an element of $L(G)$ and write $X \in \mathbb{X}$ to indicate that the local 1-ps $X$ is a representative of the class $\mathbb{X}$. We have a scalar multiplication map $(s, \mathbb{X}) \mapsto s \cdot \mathbb{X}: \mathbb{R} \times L(G) \rightarrow L(G)$, where $s \cdot \mathbb{X}$ is defined as follows. Let $X \in \mathbb{X}$ with $X:(-r, r) \rightarrow G$. If $s=0$, then $0 \cdot \mathbb{X}=\mathbb{O}$, where $O: \mathbb{R} \rightarrow G$ is defined by $O(t)=1$ for all $t \in \mathbb{R}$ and $\mathbb{O}=[O]$. Else, define $s X:\left(\frac{-r}{|s|}, \frac{r}{|s|}\right) \rightarrow G$ by $(s X)(t)=X(s t)$. Then $s X$ is a local 1-ps of $G$, and we set $s \cdot \mathbb{X}=[s X]$. It is easy to verify that for all $\mathbb{X} \in L(G)$ and $s, s^{\prime} \in \mathbb{R}$, $1 \cdot \mathbb{X}=\mathbb{X}$ and $s \cdot\left(s^{\prime} \cdot \mathbb{X}\right)=\left(s s^{\prime}\right) \cdot \mathbb{X}$.

Suppose $X_{1}$ and $X_{2}$ are local 1-parameter subgroups of $G$ with $\left[X_{1}\right]=\left[X_{2}\right]$. Suppose also that $t \in \operatorname{domain}\left(X_{1}\right) \cap \operatorname{domain}\left(X_{2}\right)$. Then $X_{1}(t)=X_{2}(t)$. To see this, let $r \in \mathbb{R}^{>0}$ be such that $X_{1}\left|(-r, r)=X_{2}\right|(-r, r)$ and choose $n>0$ such that $\frac{t}{n} \in(-r, r)$. Then $X_{1}(t)=\left(X_{1}\left(\frac{t}{n}\right)\right)^{n}=\left(X_{2}\left(\frac{t}{n}\right)\right)^{n}=X_{2}(t)$. Hence, it makes sense to define, for $\mathbb{X} \in L(G)$,

$$
\operatorname{domain}(\mathbb{X}):=\bigcup_{X \in \mathbb{X}} \operatorname{domain}(X)
$$

and for $t \in \operatorname{domain}(\mathbb{X})$, we will write $\mathbb{X}(t)$ to denote $X(t)$ for any $X \in \mathbb{X}$ with $t \in \operatorname{domain}(X)$.

\section{The Category of Local Groups}

In this subsection, we define the analogs of a few ordinary group theoretic notions in the local group setting. We will not need most of this material until Section 8. The presentation given here borrows from [12] and [16].

Definition 2.8. Suppose $G=(G, 1, \iota, p)$ and $G^{\prime}=\left(G^{\prime}, 1^{\prime}, \iota^{\prime}, p^{\prime}\right)$ are local groups with $\operatorname{domain}(\iota)=\Lambda$, domain $(p)=\Omega$, domain $\left(\iota^{\prime}\right)=\Lambda^{\prime}$ and domain $\left(p^{\prime}\right)=\Omega^{\prime}$. A morphism from $G$ to $G^{\prime}$ is a continuous function $f: G \rightarrow G^{\prime}$ such that:

(1) $f(1)=1^{\prime}, f(\Lambda) \subseteq \Lambda^{\prime}$ and $(f \times f)(\Omega) \subseteq \Omega^{\prime}$,

(2) $f(\iota(x))=\iota^{\prime}(f(x))$ for $x \in \Lambda$, and

(3) $f(p(x, y))=p^{\prime}(f(x), f(y))$ for $(x, y) \in \Omega$.

Suppose $U_{1}$ and $U_{2}$ are open neighborhoods of 1 in $G$ and suppose that $f_{1}: G \mid U_{1} \rightarrow G^{\prime}$ and $f_{2}: G \mid U_{2} \rightarrow G^{\prime}$ are both morphisms. We say that 
$f_{1}$ and $f_{2}$ are equivalent if there is an open neighborhood $U_{3}$ of 1 in $G$ such that $U_{3} \subseteq U_{1} \cap U_{2}$ and $f_{1}\left|U_{3}=f_{2}\right| U_{3}$. This defines an equivalence relation on the set of morphisms $f: G \mid U \rightarrow G^{\prime}$, where $U$ ranges over all open neighborhoods of 1 in $G$. We will call the equivalence classes local morphisms from $G$ to $G^{\prime}$. To indicate that $f$ is a local morphism from $G$ to $G^{\prime}$, we write " $f: G \rightarrow G^{\prime}$ is a local morphism," even though $f$ is not a function from $G$ to $G^{\prime}$, but rather an equivalence class of certain partial functions.

Suppose $G, G^{\prime}, G^{\prime \prime}$ are local groups and $f: G \rightarrow G^{\prime}$ and $g: G^{\prime} \rightarrow G^{\prime \prime}$ are local morphisms. Choose a representative of $f$ whose image lies in the domain of a representative of $g$. Then these representatives can be composed and the composition is a morphism. We denote by $g \circ f$ the equivalence class of this composition. The equivalence class of the identity function on $G$ is a local morphism and will be denoted by $\operatorname{id}_{G}$. We thus have the category LocGrp whose objects are the local groups and whose arrows are the local morphisms with the composition defined above. A local isomorphism is an isomorphism in the category LocGrp. Hence, if $f: G \rightarrow G^{\prime}$ is a local isomorphism, then there is a representative $f: G\left|U \rightarrow G^{\prime}\right| U^{\prime}$, where $U$ and $U^{\prime}$ are open neighborhoods of 1 and $1^{\prime}$ in $G$ and $G^{\prime}$ respectively, $f: U \rightarrow U^{\prime}$ is a homeomorphism, and $f: G\left|U \rightarrow G^{\prime}\right| U^{\prime}$ and $f^{-1}: G^{\prime}\left|U^{\prime} \rightarrow G\right| U$ are morphisms. If there exists a local isomorphism between the local groups $G$ and $G^{\prime}$, then we say that $G$ and $G^{\prime}$ are locally isomorphic.

Example 2.9. One can consider the additive group $\mathbb{R}$ as a local group and $(-r, r)$ for $r \in(0, \infty]$ as a restriction of this local group, so that a local 1-ps $(-r, r) \rightarrow G$ is a morphism of local groups. In this way, the elements of $L(G)$ are local morphisms from $\mathbb{R}$ to $G$. Moreover, given any local morphism $f: G \rightarrow G^{\prime}$, one gets a map of sets $L(f): L(G) \rightarrow L\left(G^{\prime}\right)$ given by $L(f)(\mathbb{X})=f \circ \mathbb{X}$. It can easily be checked that this yields a functor $L:$ LocGrp $\rightarrow$ Sets.

Definition 2.10. A sublocal group of $G$ is a set $H \subseteq G$ containing 1 for which there exists an open neighborhood $V$ of 1 in $G$ such that

(1) $H \subseteq V$ and $H$ is closed in $V$;

(2) if $x \in H \cap \Lambda$ and $x^{-1} \in V$, then $x^{-1} \in H$;

(3) if $(x, y) \in(H \times H) \cap \Omega$ and $x y \in V$, then $x y \in H$.

With $H$ and $V$ as above, we call $H$ a sublocal group of $G$ with associated neighborhood $V$. A normal sublocal group of $G$ is a sublocal group $H$ of $G$ with an associated neighborhood $V$ such that $V$ is symmetric and

(4) if $y \in V$ and $x \in H$ are such that $y x y^{-1}$ is defined and $y x y^{-1} \in V$, then $y x y^{-1} \in H$. 
For such $H$ and $V$, we also say that $H$ is a normal sublocal group of $G$ with associated normalizing neighborhood $V$.

One checks easily that if $H$ is a sublocal group of $G$ with associated neighborhood $V$, then

$$
\left(H, 1, \iota\left|H \cap \Lambda \cap \iota^{-1}(V), p\right|(H \times H) \cap \Omega \cap p^{-1}(V)\right)
$$

is a local group, to be denoted by $H$ for simplicity, and that then the inclusion $H \hookrightarrow G$ is a morphism. Sublocal groups $H$ and $H^{\prime}$ of $G$ are equivalent if there is an open neighborhood $U$ of 1 in $G$ such that $H \cap U=H^{\prime} \cap U$.

Example 2.11. Suppose $f: G \rightarrow G^{\prime}$ is a morphism and $\Lambda=G$. Then $\operatorname{ker}(f):=f^{-1}(\{1\})$ is a normal sublocal group of $G$ with associated normalizing neighborhood $G$.

The following lemma has a routine verification.

Lemma 2.12. Suppose $H$ is a normal sublocal group of $G$ with associated normalizing neighborhood $V$. Suppose $U \subseteq H$ is open in $H$ and symmetric. Let $U^{\prime} \subseteq V$ be a symmetric open neighborhood of 1 in $G$ such that $U=H \cap$ $U^{\prime}$. Then $H \mid U$ is a normal sublocal group of $G$ with associated normalizing neighborhood $U^{\prime}$.

We now investigate quotients in this category. For the rest of this subsection, assume that $H$ is a normal sublocal group of $G$ with associated normalizing neighborhood $V$.

Lemma 2.13. Let $W$ be a symmetric open neighborhood of 1 in $G$ such that $W \subseteq \mathcal{U}_{6}$ and $W^{6} \subseteq V$. Then

(1) The binary relation $E_{H}$ on $W$ defined by

$$
E_{H}(x, y) \text { if and only if } x^{-1} y \in H
$$

is an equivalence relation on $W$.

(2) For $x \in W$, let $x H:=\{x h \mid h \in H\}$. Then for $x, y \in W, E_{H}(x, y)$ holds if and only if $(x H) \cap W=(y H) \cap W$. In other words, if $E_{H}(x)$ denotes the equivalence class of $x$, then $E_{H}(x)=(x H) \cap W$. We call the equivalence classes local cosets of $H$.

Proof. For (1), first note that the reflexivity of $E_{H}$ is trivial. Now suppose $E_{H}(x, y)$ holds, i.e. that $x^{-1} y \in H$. Since $\left(x^{-1} y\right)^{-1} \in V$, we have that $\left(x^{-1} y\right)^{-1} \in H$. It can easily be verified that $\left(x^{-1} y\right)^{-1}=y^{-1} x$, so $E_{H}(y, x)$ holds. Finally, suppose $E_{H}(x, y)$ and $E_{H}(y, z)$ hold. It follows 
from $\left(x^{-1} y\right)\left(y^{-1} z\right) \in V$ that $\left(x^{-1} y\right)\left(y^{-1} z\right) \in H$. However, since $x, y, z \in \mathcal{U}_{4}$, we know that $\left(x^{-1} y\right)\left(y^{-1} z\right)=x^{-1} z$, so we have transitivity and (1) is proven.

For (2), first suppose that $E_{H}(x, y)$ holds. Let $w \in(y H) \cap W$. Let $h \in H$ be such that $w=y h$. Then $h=y^{-1} w \in W^{2}$. Since $\left(x^{-1} y\right) h \in V$, we have $\left(x^{-1} y\right) h \in H$. But $\left(x^{-1} y\right) h=x^{-1} w$, so $w \in(x H) \cap W$. By symmetry, $(x H) \cap W=(y H) \cap W$. For the converse, suppose $(x H) \cap W=(y H) \cap W$. Since $y \in(y h) \cap W$, we have $y \in(x H) \cap W$. Let $h \in H$ be such that $y=x h$. Then since $x h \in W$, we know that $x^{-1}(x h)$ is defined. We thus have, by (A), $x^{-1} y=h \in H$ and so $E_{H}(x, y)$ holds.

Let $\pi_{H, W}: W \rightarrow W / E_{H}$ be the canonical map. Give $W / E_{H}$ the quotient topology. Then $\pi_{H, W}$ becomes an open continuous map.

Let $\iota_{H, W}: W / E_{H} \rightarrow W / E_{H}$ be defined by $\iota_{H, W}\left(E_{H}(x)\right)=E_{H}\left(x^{-1}\right)$. Let $\Omega_{H, W}:=\left(\pi_{H, W} \times \pi_{H, W}\right)\left((W \times W) \cap p^{-1}(W)\right)$. It is easy to check that one can define a map $p_{H, W}: \Omega_{H, W} \rightarrow W / E_{H}$ by $p_{H, W}\left(E_{H}(x), E_{H}(y)\right)=E_{H}(x y)$, where $x$ and $y$ are representatives of their respective local cosets chosen so that $x y \in W$. The following lemma is easy to verify.

Lemma 2.14. With the notations as above,

$$
(G / H)_{W}:=\left(W / E_{H}, E_{H}(1), \iota_{H, W}, p_{H, W}\right)
$$

is a local group and $\pi_{H, W}: G \mid W \rightarrow(G / H)_{W}$ is a morphism.

In the construction of quotients, we have made some choices. We would expect that, locally, we have the same local group. The following lemma expresses this.

Lemma 2.15. Suppose that $H^{\prime}$ is also a normal sublocal group of $G$ with associated normalizing neighborhood $V^{\prime}$ such that $H$ is equivalent to $H^{\prime}$. Let $W^{\prime}$ be a symmetric open neighborhood of 1 in $G$ used to construct $\left(G / H^{\prime}\right)_{W^{\prime}}$. Then $(G / H)_{W}$ and $\left(G / H^{\prime}\right)_{W}$ are locally isomorphic.

Proof. Let $U$ be an open neighborhood of 1 in $G$ such that $H \cap U=H^{\prime} \cap U$. Choose open $U_{1}$ containing 1 such that $U_{1}^{2} \subseteq U$. Let $U_{2}:=W \cap W^{\prime} \cap U_{1}$. Consider the map $\psi: \pi_{H, W}\left(U_{2}\right) \rightarrow W^{\prime} / E_{H^{\prime}}$ given by $\psi\left(E_{H}(x)\right)=E_{H^{\prime}}(x)$ for $x \in U_{2}$. One can verify that $\psi:(G / H)_{W} \mid \pi_{H, W}\left(U_{2}\right) \rightarrow\left(G / H^{\prime}\right)_{W^{\prime}}$ induces the desired local isomorphism.

From now on, we write $G / H$ to denote $(G / H)_{W}$, where $W$ is any open neighborhood of 1 as in Lemma 2.13. We will also write $\pi: G \rightarrow G / H$ to denote the local morphism induced by $\pi_{H, W}: G \mid W \rightarrow(G / H)_{W}$. Since all of 
the various quotients defined in this way are locally isomorphic, fixing one's attention on one particular local coset space is no loss of generality for our purposes.

\section{Further Properties of Local Groups}

In this subsection, we first show that we can place two further assumptions on our local groups which lead to no loss of generality in the solution of the Local H5. Under these new assumptions, we derive several properties of the notion " $a_{1} \cdots a_{n}$ is defined."

Let $U:=\Lambda \cap \iota^{-1}(\Lambda)$, an open neighborhood of 1 in $G$. Note that if $g \in U$, then $\iota(g) \in \Lambda$ and $\iota(\iota(g))=g \in \Lambda$, implying that $\Lambda_{U}=U$.

From this point on, we assume that $\Lambda=G$ for every local group $G$ in this paper. By the preceding remarks, every local group has a restriction satisfying this condition and so this is no loss of generality for our purpose. Note that, with these assumptions, if $(x, y) \in \Omega$ and $x y=1$, then $x=y^{-1}$ and $y=x^{-1}$. In particular, $\left(x^{-1}\right)^{-1}=1$ for all $x \in G$, and $G$ is symmetric.

The following lemma is obvious for topological groups, but requires some care in the local group setting.

Lemma 2.16. (Homogeneity)

(1) For any $g \in G$, there are open neighborhoods $V$ and $W$ of 1 and $g$ respectively such that $\{g\} \times V \subseteq \Omega, g V \subseteq W,\left\{g^{-1}\right\} \times W \subseteq \Omega$, $g^{-1} W \subseteq V$, and the maps

$$
v \mapsto g v: V \rightarrow W \text { and } w \mapsto g^{-1} w: W \rightarrow V
$$

are each others inverses (and hence homeomorphisms).

(2) $G$ is locally compact if and only if there is a compact neighborhood of 1 .

Proof. Clearly (1) implies (2). For any $g \in G$, define

$$
\Omega_{g}:=\{h \in G \mid(g, h) \in \Omega\} .
$$

Then $\Omega_{g}$ is an open subset of $G$ and $L_{g}: \Omega_{g} \rightarrow G$, defined by $L_{g}(h)=g h$, is continuous. Let $V:=\left(L_{g}\right)^{-1}\left(\Omega_{g^{-1}}\right)$. Then $V$ is open and $1 \in V$. Let $W=L_{g}(V) \subseteq \Omega_{g^{-1}}$. Then $W$ is open since $W=L_{g^{-1}}^{-1}(V)$. From our choices we can check that $L_{g} \mid V$ and $L_{g^{-1}} \mid W$ are inverses of each other. 
Corollary 2.17. Let $U=\mathcal{U}_{3}$. Then for any $g, h \in U$ such that $(g, h) \in \Omega_{U}$, one has $\left(h^{-1}, g^{-1}\right) \in \Omega_{U}$ and $(g h)^{-1}=h^{-1} g^{-1}$.

Proof. Suppose $g, h \in U$ with $(g, h) \in \Omega_{U}$. Then we have $h^{-1}, g^{-1}, g h \in U$. Hence $h^{-1} \cdot g^{-1} \cdot(g h)$ is defined and

$$
h^{-1} \cdot g^{-1} \cdot(g h)=h^{-1} \cdot\left(g^{-1} \cdot(g h)\right)=h^{-1} h=1
$$

by (A). But $h^{-1} \cdot g^{-1} \cdot(g h)=\left(h^{-1} g^{-1}\right) \cdot(g h)$, so $h^{-1} g^{-1}=(g h)^{-1}$. Also, since $h^{-1} g^{-1}=(g h)^{-1} \in U$, we have $\left(h^{-1}, g^{-1}\right) \in \Omega_{U}$.

From now on, we make the following further assumption on our local group $G$ :

$$
\text { if }(g, h) \in \Omega \text {, then }\left(h^{-1}, g^{-1}\right) \in \Omega \text { and }(g h)^{-1}=h^{-1} g^{-1} .
$$

By the preceding corollary, every local group has a restriction satisfying this assumption and so this is no loss of generality for our purpose.

Lemma 2.18. Let $a, a_{1}, \ldots, a_{n} \in G$. Then

(1) If $a_{1} \cdots a_{n}$ is defined and $1 \leq i \leq j \leq n$, then $a_{i} \cdots a_{j}$ is defined. In particular, if $a^{n}$ is defined and $m \leq n$, then $a^{m}$ is defined.

(2) If $a^{m}$ is defined and $i, j \in\{0, \ldots, m\}$ are such that $i+j=m$, then $\left(a^{i}, a^{j}\right) \in \Omega$ and $a^{i} \cdot a^{j}=a^{m}$.

(3) If $a^{n}$ is defined and, for all $i, j \in\{1, \ldots, n\}$ with $i+j=n+1$, one has $\left(a^{i}, a^{j}\right) \in \Omega$, then $a^{n+1}$ is defined. More generally, if $a_{1} \cdots a_{n}$ is defined, $a_{i} \cdots a_{n+1}$ is defined for all $i \in\{2, \ldots, n\}$ and

$$
\left(a_{1} \cdots a_{i}, a_{i+1} \cdots a_{n+1}\right) \in \Omega \text { for all } i \in\{1, \ldots, n\},
$$

then $a_{1} \cdots a_{n+1}$ is defined.

(4) If $a^{n}$ is defined, then $\left(a^{-1}\right)^{n}$ is defined and $\left(a^{-1}\right)^{n}=\left(a^{n}\right)^{-1}$. (In this case, we denote $\left(a^{-1}\right)^{n}$ by $a^{-n}$.) More generally, if $a_{1} \cdots a_{n}$ is defined, then $a_{n}^{-1} \cdots a_{1}^{-1}$ is defined and $\left(a_{1} \cdots a_{n}\right)^{-1}=a_{n}^{-1} \cdots a_{1}^{-1}$.

(5) If $k, l \in \mathbb{Z}, l \neq 0$, and $a^{k \cdot l}$ is defined, then $a^{k}$ is defined, $\left(a^{k}\right)^{l}$ is defined and $\left(a^{k}\right)^{l}=a^{k \cdot l}$.

Proof. The proofs of (1) and (2) are immediate from the definitions. (3) follows from repeated uses of (A). We prove the first assertion of (4) by induction on $n$. The cases $n=1$ and $n=2$ are immediate from our assumptions on local groups. For the induction step, suppose $a^{n+1}$ is defined and $i, j \in\{1, \ldots, n\}$ with $i+j=n+1$. Then since $\left(a^{j}, a^{i}\right) \in \Omega$, by induction we have $\left(\left(a^{-1}\right)^{i},\left(a^{-1}\right)^{j}\right) \in \Omega$. Hence, by (3) of the lemma, we know that $\left(a^{-1}\right)^{n+1}$ is defined. Also,

$$
\left(a^{-1}\right)^{n+1}=\left(a^{-1}\right)^{n} \cdot a^{-1}=\left(a^{n}\right)^{-1} \cdot a^{-1}=\left(a \cdot a^{n}\right)^{-1}=\left(a^{n+1}\right)^{-1} .
$$

The second assertion of (4) is proved in the same manner. 
We now prove (5). It is easy to see that it is enough to check the assertion for $k, l \in \mathbb{N}, l \neq 0$. Since $k \leq k \cdot l$, we have $a^{k}$ is defined by (1). We prove the rest by induction on $l$. This is clear for $l=1$. Suppose the assertion is true for all $i \leq l$ and suppose $a^{k \cdot(l+1)}$ is defined To see that $\left(a^{k}\right)^{l+1}$ is defined, we must check that $\left(\left(a^{k}\right)^{i},\left(a^{k}\right)^{j}\right) \in \Omega$ for $i, j \in\{1, \ldots, l\}$ such that $i+j=l+1$. This is the case by (2), since for such $i, j$, we have $k \cdot i+k \cdot j=k \cdot(l+1)$ and by induction $\left(a^{k}\right)^{i}=a^{k \cdot i}$ and $\left(a^{k}\right)^{j}=a^{k \cdot j}$. Now that we know that $\left(a^{k}\right)^{l+1}$ is defined, we must have, by induction, that

$$
\left(a^{k}\right)^{l+1}=\left(a^{k}\right)^{l} \cdot a^{k}=a^{k \cdot l} \cdot a^{k}=a^{k \cdot l+k} .
$$

By (4) of the previous lemma, we can now speak of $a^{k}$ being defined for any $k \in \mathbb{Z}$, where $a^{k}$ being defined for $k<0$ means that $a^{-k}$ is defined.

Corollary 2.19. Suppose $i, j \in \mathbb{Z}$ and $i \cdot j<0$. If $a^{i}$ and $a^{j}$ are defined and $\left(a^{i}, a^{j}\right) \in \Omega$, then $a^{i+j}$ is defined and $a^{i} \cdot a^{j}=a^{i+j}$.

Proof. The fact that $a^{i+j}$ is defined is clear from the preceding lemma. Next note that the result is trivial if $i=-j$. We only prove the case that $i>0$ and $j<0$ and $i>|j|$, the other cases being similar. By the preceding lemma, we have $a^{i}=a^{i+j} \cdot a^{-j}$, whence $a^{i} \cdot a^{j}=a^{i+j}$ by $(\mathrm{A})$.

\section{The Nonstandard Setting}

We assume familiarity with this setting; see [2] and [5] for details. Here we just fix notations and terminology. To each relevant "basic" set $S$ corresponds functorially a set $S^{*} \supseteq S$, the nonstandard extension of $S$. In particular, $\mathbb{N}, \mathbb{R}, G$ extend to $\mathbb{N}^{*}, \mathbb{R}^{*}, G^{*}$, respectively. Also, any (relevant) relation $R$ and function $F$ on these basic sets extends functorially to a relation $R^{*}$ and function $F^{*}$ on the corresponding nonstandard extensions of these basic sets. For example, the linear ordering $<$ on $\mathbb{N}$ extends to a linear ordering $<^{*}$ on $\mathbb{N}^{*}$, and the local group operation $p: \Omega \rightarrow G$ of $G$ extends to an operation $p^{*}: \Omega^{*} \rightarrow G^{*}$. For the sake of readability we only use a star in denoting the nonstandard extension of a basic set, but drop the star when indicating the nonstandard extension of a relation or function on these basic sets. For example, when $x, y \in \mathbb{R}^{*}$ we write $x+y$ and $x<y$ rather than $x+{ }^{*} y$ and $x<{ }^{*} y$.

Given an ambient hausdorff space $S$ and $s \in S$, the monad of $s$, notation: $\boldsymbol{\mu}(s)$, is by definition the intersection of all $U^{*} \subseteq S^{*}$ with $U$ a neighborhood of $s$ in $S$; the elements of $\boldsymbol{\mu}(s)$ are the points of the nonstandard space $S^{*}$ that are infinitely close to $s$. The points of $S^{*}$ that are infinitely close to 
some $s \in S$ are called nearstandard, and $S_{\mathrm{ns}}^{*}$ is the set of nearstandard points of $S^{*}$ :

$$
S_{\mathrm{ns}}^{*}=\bigcup_{s \in S} \boldsymbol{\mu}(s)
$$

Since $S$ is hausdorff, $\boldsymbol{\mu}(s) \cap \boldsymbol{\mu}\left(s^{\prime}\right)=\emptyset$ for distinct $s, s^{\prime} \in S$. Thus, for $s^{*} \in S_{\text {ns }}^{*}$, we can define the standard part of $s^{*}$, notation: st $\left(s^{*}\right)$, to be the unique $s \in S$ such that $s^{*} \in \boldsymbol{\mu}(s)$. For $s_{1}, s_{2} \in S_{\text {ns }}^{*}$, we put $s_{1} \sim s_{2}$ iff $\operatorname{st}\left(s_{1}\right)=\operatorname{st}\left(s_{2}\right)$.

We let $\boldsymbol{\mu}:=\boldsymbol{\mu}(1)$ be the monad of 1 in $G^{*}$; note that $\boldsymbol{\mu}$ is a group with product map $(x, y) \mapsto x y:=p(x, y)$.

From now on, we let $\nu, \sigma, \tau, \eta, N$ range over $\mathbb{N}^{*}$ and $i$ and $j$ range over $\mathbb{Z}^{*}$, but $m$ and $n$ will always denote elements of $\mathbb{N}$. We use the Landau notation as follows: $i=\mathrm{O}(\nu)$ means $|i|<\frac{\nu}{n}$ for all $n>0$ and $i=\mathrm{O}(\nu)$ means $|i|<n \nu$ for some $n>0$.

In our nonstandard arguments, we will assume as much saturation as needed. The following lemma uses routine nonstandard methods and is left for the reader to verify.

Lemma 2.20. $G$ is NSS if and only if there are no internal subgroups of $\boldsymbol{\mu}$ other than $\{1\}$.

We also consider the internal versions of the notions and results that appear earlier in this section. In particular, if $\left(a_{1}, \ldots, a_{\nu}\right)$ is an internal sequence in $G^{*}$, then it makes sense to say that $\left(a_{1}, \ldots, a_{\nu}\right)$ (internally) represents $b$, where $b \in G^{*}$. We say that $a_{1} \cdots a_{\nu}$ is defined if there exists $b \in G^{*}$ such that $\left(a_{1}, \ldots, a_{\nu}\right)$ represents $b$. Likewise, we have the notion " $a$ is defined" for $a \in G^{*}, i \in \mathbb{Z}^{*}$.

The proof of the following lemma is trivial.

\section{Lemma 2.21.}

(1) Suppose $a, b \in G$ and $a^{\prime} \in \boldsymbol{\mu}(a)$ and $b^{\prime} \in \boldsymbol{\mu}(b)$. If $(a, b) \in \Omega$, then $\left(a^{\prime}, b^{\prime}\right) \in \Omega^{*}, a^{\prime} \cdot b^{\prime} \in G_{\mathrm{ns}}^{*}$, and $\operatorname{st}\left(a^{\prime} \cdot b^{\prime}\right)=a \cdot b$.

(2) For any $a \in G_{\mathrm{ns}}^{*}$ and $b \in \boldsymbol{\mu},(a, b),(b, a) \in \Omega^{*}, a \cdot b, b \cdot a \in G_{\mathrm{ns}}^{*}$, and $\operatorname{st}(a \cdot b)=\operatorname{st}(b \cdot a)=\operatorname{st}(a)$.

(3) For any $a, b \in G_{\mathrm{ns}}^{*}$, if $\left(a, b^{-1}\right) \in \Omega^{*}$ and $a b^{-1} \in \boldsymbol{\mu}$, then $a \sim b$.

(4) For any $a \in G, a^{\prime} \in \boldsymbol{\mu}(a)$, and any $n$, if $a^{n}$ is defined, then $\left(a^{\prime}\right)^{n}$ is defined and $\left(a^{\prime}\right)^{n} \in \boldsymbol{\mu}\left(a^{n}\right)$. 
A standard consequence of the last item of the preceding lemma is that the partial function $p_{n}: G \rightarrow G$, defined by $p_{n}(a)=a^{n}$ if $a^{n}$ is defined, has an open domain and is continuous. Note that $\mathcal{U}_{n} \subseteq$ domain $\left(p_{n}\right)$.

Lemma 2.22. Suppose $U$ is a neighborhood of 1 in $G$ and $a \in \boldsymbol{\mu}$. Then there is a $\nu>\mathbb{N}$ such that $a^{\sigma}$ is defined and $a^{\sigma} \in U^{*}$ for all $\sigma \in\{1, \ldots, \nu\}$.

Proof. Let $X=\left\{\sigma \in \mathbb{N}^{*} \mid a^{\sigma}\right.$ is defined and $\left.a^{\sigma} \in U^{*}\right\}$. Then $X$ is an internal subset of $\mathbb{N}^{*}$ which contains $\mathbb{N}$ since $\boldsymbol{\mu}$ is a subgroup of $G^{*}$. Hence, by overflow, there is a $\nu>\mathbb{N}$ such that $\{0,1, \ldots, \nu\} \subseteq X$.

\section{Connectedness And Purity}

In this section, we describe the different ways powers of infinitesimals can grow and its impact on the existence of connected subgroups of $G$. We also show how to build local 1-parameter subgroups from "pure" infinitesimals.

In the rest of this paper, we assume that $G$ is locally compact. In particular, $G$ is regular as a topological space. Also, $a$ and $b$ will denote elements of $G^{*}$.

For $\nu>\mathbb{N}$, we set:

$$
\begin{aligned}
G(\nu) & :=\left\{a \in \boldsymbol{\mu} \mid a^{i} \text { is defined and } a^{i} \in \boldsymbol{\mu} \text { for all } i=\mathrm{o}(\nu)\right\}, \\
G^{\mathrm{o}}(\nu) & :=\left\{a \in \boldsymbol{\mu} \mid a^{i} \text { is defined and } a^{i} \in \boldsymbol{\mu} \text { for all } i=\mathrm{O}(\nu)\right\} .
\end{aligned}
$$

Note that $G^{\circ}(\nu) \subseteq G(\nu)$ and both sets are symmetric.

Lemma 3.1. Let $a \in \boldsymbol{\mu}$ and $\nu>\mathbb{N}$. Then the following are equivalent:

(1) $a^{i}$ is defined and $a^{i} \in \boldsymbol{\mu}$ for all $i \in\{1, \ldots, \nu\}$;

(2) $a \in G^{\mathrm{o}}(\nu)$;

(3) there is $\tau \in\{1, \ldots, \nu\}$ such that $\nu=\mathrm{O}(\tau)$ and $a^{i}$ is defined and $a^{i} \in \boldsymbol{\mu}$ for all $i \in\{1, \ldots, \tau\}$.

Proof. (1) $\Rightarrow(2)$ : Fix $\sigma$ such that $\sigma=\mathrm{O}(\nu)$. We claim that if $a^{\sigma}$ is defined, then $a^{\sigma} \in \boldsymbol{\mu}$. To see this, write $\sigma=n \nu+\eta$ for some $n$ and $\eta<\nu$. By Lemma 2.18,

$$
a^{\sigma}=\left(a^{\nu}\right)^{n} \cdot a^{\eta} \in \boldsymbol{\mu} \cdot \boldsymbol{\mu} \subseteq \boldsymbol{\mu} .
$$

We now prove by internal induction that $a^{i}$ is defined for all $i \in\{1, \ldots, \sigma\}$. The assertion is true for $i=1$ and inductively suppose that $a^{i}$ is defined and $i+1 \leq \nu$. By Lemma 2.18, in order to show that $a^{i+1}$ is defined, it suffices to show that $\left(a^{k}, a^{l}\right) \in \Omega^{*}$ for all $k, l \in\{1, \ldots, i\}$ with $k+l=i+1$. However, $\left(a^{k}, a^{l}\right) \in \boldsymbol{\mu} \times \boldsymbol{\mu} \subseteq \Omega^{*}$, finishing the induction. 
Clearly $(2) \Rightarrow(1)$ and $(3)$, so it remains to prove that $(3) \Rightarrow(2)$. So assume that there is $\tau \in\{1, \ldots, \nu\}$ as in (3). By the proof of $(1) \Rightarrow(2)$, we see that $a \in G^{\mathrm{o}}(\tau)$. Since $G^{\mathrm{o}}(\tau) \subseteq G^{\mathrm{o}}(\nu)$, we are finished.

Definition 3.2. We say that $a \in \boldsymbol{\mu}$ is degenerate if, for all $i, a^{i}$ is defined and $a^{i} \in \boldsymbol{\mu}$.

It is easy to see that $G$ is NSS if and only if $\boldsymbol{\mu}$ has no nondegenerate elements other than 1.

Lemma 3.3. Let $a_{1}, \ldots, a_{\nu}$ be an internal sequence of elements of $G^{*}$ with $\nu>0$ such that for all $i \in\{1, \ldots, \nu\}$ we have $a_{i} \in \boldsymbol{\mu}, a_{1} \cdots a_{i}$ is defined and $a_{1} \cdots a_{i} \in G_{\mathrm{ns}}^{*}$. Then the set

$$
S:=\left\{\operatorname{st}\left(a_{1} \cdots a_{i}\right): 1 \leq i \leq \nu\right\} \subseteq G
$$

is compact and connected (and contains 1).

The proof is as in the global case, but we include it for completeness.

Proof. Suppose for every compact $K \subseteq G$, there is $i \in\{1, \ldots, \nu\}$ such that $a_{1} \cdots a_{i} \notin K^{*}$. Then, by saturation, there is $i \in\{1, \ldots, \nu\}$ such that $a_{1} \cdots a_{i} \notin K^{*}$ for all compact $K \subseteq G$, contradicting local compactness. Hence there is compact $K \subseteq G$ with $a_{1} \cdots a_{i} \in K^{*}$ for all $i \in\{1, \ldots, \nu\}$, so $S \subseteq K$. It is well-known that $S$ must be closed, being the standard part of an internal nearstandard set. Hence $S$ is compact.

Suppose $S$ is not connected. Then we have disjoint open subsets $U$ and $V$ of $G$ such that $S \subseteq U \cup V$ and $S \cap U \neq \emptyset, S \cap V \neq \emptyset$. Assume $1 \in U$, so $a_{1} \in U^{*}$. Choose $i \in\{1, \ldots, \nu\}$ minimal such that $a_{1} \cdots a_{i} \in V^{*}$. Then $i \geq 2$ and $a_{1} \cdots a_{i-1} \in U^{*}$. Now $a:=\operatorname{st}\left(a_{1} \cdots a_{i-1}\right)=\operatorname{st}\left(a_{1} \cdots a_{i}\right) \in S$. If $a \in U$, then $a_{1} \cdots a_{i-1} \in U^{*}$ and if $a \in V$, then $a_{1} \cdots a_{i} \in V^{*}$; either option yields a contradiction.

Until further notice, $U$ denotes a compact symmetric neighborhood of 1 such that $U \subseteq \mathcal{U}_{2}$.

Definition 3.4. Let $a \in G^{*}$. If, for all $i, a^{i}$ is defined and $a^{i} \in U^{*}$, define $\operatorname{ord}_{U}(a)=\infty$. Else, define $\operatorname{ord}_{U}(a)=\nu$ if, for all $i$ with $|i| \leq \nu, a^{i}$ is defined, $a^{i} \in U^{*}$, and $\nu$ is the largest element of $\mathbb{N}^{*}$ for which this happens.

It is easy to see that $\operatorname{ord}_{U}(a)=0$ iff $a \notin U^{*}$. By Lemma 2.22, we can see that $\operatorname{ord}_{U}(a)>\mathbb{N}$ if $a \in \boldsymbol{\mu}$. Note that if $a \in \boldsymbol{\mu}$ and $\operatorname{ord}_{U}(a)=\nu \in \mathbb{N}^{*}$, 
then $a^{\nu+1}$ is defined. To see this, it is enough to check that $\left(a^{i}, a^{j}\right) \in \Omega^{*}$ for all $i, j \in\{1, \ldots, \nu\}$ such that $i+j=\nu+1$. However, this is true since $\left(a^{i}, a^{j}\right) \in U^{*} \times U^{*} \subseteq \mathcal{U}_{2}^{*} \times \mathcal{U}_{2}^{*} \subseteq \Omega^{*}$. Hence, if $\operatorname{ord}_{U}(a)=\nu$, then this is not because $a^{\nu+1}$ is undefined but rather for the more important reason that $a^{\nu+1}$ is defined but not in $U^{*}$. We have thus captured the correct adaptation of the global notion to our local setting.

Lemma 3.5. Suppose $a \in \boldsymbol{\mu}$ and $a^{i} \notin \boldsymbol{\mu}$ for some $i=\mathrm{o}\left(\operatorname{ord}_{U}(a)\right)$. Then $U$ contains a nontrivial connected subgroup of $G$.

Proof. By the previous lemma, the set

$$
G_{U}(a):=\left\{\operatorname{st}\left(a^{i}\right)|| i \mid=\mathrm{o}\left(\operatorname{ord}_{U}(a)\right)\right\}
$$

is a union of connected subsets of $U$, each containing 1 , hence is connected. It is also a subgroup of $G$ by Lemma 2.18 and Corollary 2.19.

Definition 3.6. We say that $a \in \boldsymbol{\mu}$ is $U$-pure $\mathrm{e}^{1}$ if it is nondegenerate and $a \in G(\tau)$, where $\tau:=\operatorname{ord}_{U}(a)$. We say that $a \in \boldsymbol{\mu}$ is pure if it is $V$-pure for some compact symmetric neighborhood $V$ of 1 such that $V \subseteq \mathcal{U}_{2}$.

The last lemma now reads that if $U$ contains no non-trivial connected subgroup of $G$, then every $a \in \boldsymbol{\mu}$ which is nondegenerate is $U$-pure.

Lemma 3.7. Let $a \in \boldsymbol{\mu}$. Then $a$ is pure iff there is $\nu>\mathbb{N}$ such that $a^{\nu}$ is defined, $a^{\nu} \notin \boldsymbol{\mu}$ and $a \in G(\nu)$.

Proof. First suppose that $a$ is $V$-pure. Let $\nu=\operatorname{ord}_{V}(a)$. Then certainly $a^{\nu}$ is defined. Now since $a^{\nu+1}$ is defined, $a^{\nu} \notin \boldsymbol{\mu}$, else $a^{\nu+1}=a^{\nu} \cdot a \in \boldsymbol{\mu}$, contradicting $a^{\nu+1} \notin V^{*}$. However, $a^{i} \in \boldsymbol{\mu}$ for $i=\mathrm{o}(\nu)$ by definition of $V$ purity. Conversely, suppose one has $\nu>\mathbb{N}$ such that $a^{\nu}$ is defined, $a^{\nu} \notin \boldsymbol{\mu}$ and $a \in G(\nu)$. Since $a^{\nu} \notin \boldsymbol{\mu}$, there is a compact symmetric neighborhood $V$ of 1 such that $V \subseteq \mathcal{U}_{2}$ and such that $a^{\nu} \notin V^{*}$. Let $\tau:=\operatorname{ord}_{V}(a)$. Then $\tau<\nu$, implying that $a \in G(\tau)$ and thus $a$ is $V$-pure.

Let $Q$ range over internal subsets of $G^{*}$ such that $Q \subseteq \boldsymbol{\mu}, 1 \in Q$, and $Q$ is symmetric. If $\nu$ is such that for every internal sequence $a_{1}, \ldots, a_{\nu}$ from $Q, a_{1} \cdots a_{\nu}$ is defined, then we define $Q^{\nu}$ to be the internal subset of $G^{*}$ consisting of all $a_{1} \cdots a_{\nu}$ for $a_{1}, \ldots, a_{\nu}$ an internal sequence from $Q$. In this situation, we say that $Q^{\nu}$ is defined.

\footnotetext{
${ }^{1}$ We have decided to replace the use of "regular" in [6] with "pure" as "regular" already has a different meaning for topological spaces.
} 
Definition 3.8. If for all $\nu, Q^{\nu}$ is defined and $Q^{\nu} \subseteq \boldsymbol{\mu}$, then we say that $Q$ is degenerate. If $Q^{\nu}$ is defined and $Q^{\nu} \subseteq U^{*}$ for all $\nu$, define $\operatorname{ord}_{U}(Q)=\infty$. Else, define $\operatorname{ord}_{U}(Q)=\nu$ if $Q^{\nu}$ is defined, $Q^{\nu} \subseteq U^{*}$, and $\nu$ is the largest element of $\mathbb{N}^{*}$ for which this happens.

Using an overflow argument similar to that in Lemma 2.22, we have that $\operatorname{ord}_{U}(Q)>\mathbb{N}$. One can also check that if $\operatorname{ord}_{U}(Q) \in \mathbb{N}^{*}$, then $Q^{\operatorname{ord}_{U}(Q)+1}$ is defined.

Lemma 3.9. Suppose $Q^{\nu} \nsubseteq \boldsymbol{\mu}$ for some $\nu=\mathrm{o}\left(\operatorname{ord}_{U}(Q)\right)$. Then $U$ contains a non-trivial connected subgroup of $G$.

Proof. As in the proof of Lemma 3.5, the set

$$
G_{U}(Q)=\left\{\operatorname{st}(a) \mid a \in Q^{\nu} \text { for some } \nu=\mathrm{o}\left(\operatorname{ord}_{U}(Q)\right)\right\}
$$

is a union of connected subsets of $U$, each containing 1 , and thus itself is a connected subset of $U$. To see that $G_{U}(Q)$ is a group, suppose that $a \in Q^{\nu}$ and $b \in Q^{\eta}$ for $\nu, \eta=\mathrm{o}\left(\operatorname{ord}_{U}(Q)\right)$. Then $\nu+\eta=\mathrm{o}\left(\operatorname{ord}_{U}(Q)\right)$ and so the concatenation of the sequences representing $a$ and $b$ represents an element of $G^{*}$, which must necessarily be $a \cdot b$. Hence $a \cdot b \in Q^{\nu+\eta}$ and $\operatorname{st}(a) \cdot \operatorname{st}(b)=\operatorname{st}(a \cdot b) \in G_{U}(Q)$. That $G_{U}(Q)$ is closed under inverses is an easy consequence of Lemma 2.18 .

Definition 3.10. We say that $Q$ is $U$-pure if $Q$ is nondegenerate and if $Q^{\nu} \subseteq \boldsymbol{\mu}$ for all $\nu=\mathrm{o}\left(\operatorname{ord}_{U}(Q)\right)$. We say that $Q$ is pure if it is $V$-pure for some compact symmetric neighborhood $V$ of 1 in $G$ such that $V \subseteq \mathcal{U}_{2}$.

The previous lemma now reads that if $U$ contains no nontrivial connected subgroups of $G$, then every nondegenerate $Q$ as above is $U$-pure.

Lemma 3.11. $Q$ is pure iff there is some $\tau>\mathbb{N}$ such that $Q^{\tau}$ is defined, $Q^{\tau} \nsubseteq \boldsymbol{\mu}$ and $Q^{\nu} \subseteq \boldsymbol{\mu}$ for all $\nu=\mathrm{o}(\tau)$.

Proof. First suppose $Q$ is $V$-pure. Let $\tau=\operatorname{ord}_{V}(Q)$. Then $\tau>\mathbb{N}, Q^{\tau}$ is defined, $Q^{\tau} \nsubseteq \boldsymbol{\mu}$ (otherwise $Q^{\tau+1} \subseteq \boldsymbol{\mu} \subseteq V^{*}$, contradicting the definition of $\tau$ ), and $Q^{\nu} \subseteq \boldsymbol{\mu}$ for $\nu=\mathrm{o}(\tau)$ by definition of being $V$-pure. Conversely, suppose we have $\tau>\mathbb{N}$ such that $Q^{\tau}$ is defined, $Q^{\tau} \nsubseteq \boldsymbol{\mu}$, and $Q^{\nu} \subseteq \boldsymbol{\mu}$ for $\nu=\mathrm{o}(\tau)$. Since $Q^{\tau} \nsubseteq \boldsymbol{\mu}$, we can find a compact symmetric neighborhood $V$ of 1 in $G$ such that $V \subseteq \mathcal{U}_{2}$ and $Q^{\tau} \nsubseteq V$. Let $\eta:=\operatorname{ord}_{V}(Q)$. Then $\eta<\tau$, implying that $Q^{\nu} \subseteq \boldsymbol{\mu}$ for all $\nu=\mathrm{o}(\eta)$, that is $Q$ is $V$-pure. 
Definition 3.12. $G$ has no small connected subgroups, henceforth abbreviated $G$ is NSCS, if there is a neighborhood of 1 in $G$ that contains no connected subgroup of $G$ other than $\{1\}$. We say that $G$ is pure if every nondegenerate $Q$ as above is pure.

Corollary 3.13. If $G$ is NSCS, then $G$ is pure.

\section{Pure infinitesimals and local 1-parameter subgroups}

Suppose $a \in \boldsymbol{\mu}$ is $U$-pure and $0<\nu=\mathrm{O}(\tau)$, where $\tau:=\operatorname{ord}_{U}(a)$. Let

$$
\Sigma_{\nu, a, U}:=\left\{r \in \mathbb{R}^{>0} \mid a^{[r \nu]} \text { is defined and } a^{i} \in U^{*} \text { if }|i| \leq[r \nu]\right\} .
$$

Since $\nu=\mathrm{O}(\tau), \Sigma_{\nu, a, U} \neq \emptyset$. Let $r_{\nu, a, U}=\sup \Sigma_{\nu, a, U}$, where we let $r_{\nu, a, U}=\infty$ if $\Sigma_{\nu, a, U}=\mathbb{R}^{>0}$. Suppose $s<r_{\nu, a, U}$. Then $s \in \Sigma_{\nu, a, U}$ and $a^{[s \nu]} \in U^{*}$. Since $a^{[s \nu]+1}$ is defined (see comment after Definition 3.4; all that was used there was that $U \times U \subseteq \Omega$ ), we have that $a^{[s \nu]+1}=a^{[s \nu]} \cdot a \in G_{\mathrm{ns}}^{*}$. Hence we have that $a^{[s \nu]}$ is defined and nearstandard for $s \in\left(-r_{\nu, a, U}, 0\right)$ as well since, for such $s$, we have that $[s \nu]=-[(-s) \nu]$ or $-[(-s) \nu]-1$.

Lemma 3.14. The map $X:\left(-r_{\nu, a, U}, r_{\nu, a, U}\right) \rightarrow G$ given by $X(s):=\operatorname{st}\left(a^{[s \nu]}\right)$ is a local 1-ps of $G$.

Proof. Throughout this proof, we let $r:=r_{\nu, a, U}$. We first show that $X$ satisfies the additivity condition in the definition of a local 1-ps of $G$. Note that the preceding arguments show that image $(X) \subseteq \mathcal{U}_{2}$. Suppose first that $s_{1}, s_{2}, s_{1}+s_{2} \in(0, r)$. Since $\left[s_{1} \nu\right]+\left[s_{2} \nu\right]=\left[\left(s_{1}+s_{2}\right) \nu\right]$ or $\left[\left(s_{1}+s_{2}\right) \nu\right]-$ 1 , we have that $a^{\left[s_{1} \nu\right]+\left[s_{2} \nu\right]}$ is defined and $\operatorname{st}\left(a^{\left[\left(s_{1}+s_{2}\right) \nu\right]}\right)=\operatorname{st}\left(a^{\left[s_{1} \nu\right]+\left[s_{2} \nu\right]}\right)$. Hence $X\left(s_{1}+s_{2}\right)=X\left(s_{1}\right) \cdot X\left(s_{2}\right)$. The case that $s_{1}, s_{2}, s_{1}+s_{2} \in(-r, r)$ with $s_{1} \cdot s_{2}<0$ works similarly, using Corollary 2.19. Using the fact that $X(-s)=X(s)^{-1}$, we get the additivity property for $s_{1}, s_{2} \in(-r, 0)$ for which $s_{1}+s_{2} \in(-r, 0)$.

It remains to show that $X$ is continuous. By Lemma 2.16, it is enough to show that $X$ is continuous at 0 . Let $V$ be a compact neighborhood of 1 in $G$. If $i=\mathrm{o}(\nu)$, then $a^{i}$ is defined and $a^{i} \in \boldsymbol{\mu} \subseteq V^{*}$. By saturation, we have $n$ such that $\frac{1}{n}<r$ and such that if $\left|\frac{i}{\nu}\right|<\frac{1}{n}$, then $a^{i}$ is defined and $a^{i} \in V^{*}$. Hence $X(s) \in V$ for $|s|<\frac{1}{n}$.

We end this section with an extension lemma which will be used in the next section. From now on, $I:=[-1,1] \subseteq \mathbb{R}$. 
Lemma 3.15. Suppose $X: I \rightarrow G$ is a continuous function such that for all $r, s \in I$, if $r+s \in I$, then $(X(r), X(s)) \in \Omega$ and $X(r+s)=X(r) \cdot X(s)$. Further suppose that $X(I) \subseteq \mathcal{U}_{2}$. Then there exists $\epsilon \in \mathbb{R}^{>0}$ and a local 1-parameter subgroup $\bar{X}:(-1-\epsilon, 1+\epsilon) \rightarrow G$ of $G$ such that $\bar{X} \mid I=X$.

Proof. Let $g:=X(1)$. Let $V$ and $W$ be open neighborhoods of 1 and $g$ respectively as in Lemma 2.16. Without loss of generality, we can suppose $V, W \subseteq \mathcal{U}_{2}$. By continuity of $X$ at 0 , we can choose $\epsilon \in(0,1)$ such that $X(-\epsilon, \epsilon) \subseteq V$. We claim that $\bar{X}:(-1-\epsilon, 1+\epsilon) \rightarrow G$ defined by $\bar{X} \mid I=X$ and for $0<\delta<\epsilon, \bar{X}(1+\delta)=X(1) \cdot X(\delta)$ and $\bar{X}(-1-\delta)=\bar{X}(1+\delta)^{-1}$ satisfies the conclusion of the lemma. Note we can define this product since all of the aforementioned elements are contained in $\mathcal{U}_{2}$. It is enough to show that $\bar{X}$ satisfies the additivity condition, for then the continuity of $\bar{X}$ at 0 will imply the continuity of $\bar{X}$ on its entire domain.

First choose $r, s \in(0,1+\epsilon)$ such that $r+s \in(0,1+\epsilon)$. The only nontrivial verificiation in this situation occurs when $r+s \in(1,1+\epsilon)$. Since not both $r$ and $s$ can be in $[1,1+\epsilon)$, we assume that $s<1$. Then since $0<r+s-1<\epsilon$, we have $\bar{X}(r+s)=\bar{X}(1+(r+s-1))=X(1) \cdot X((r+s-1))$. But $X(r+s-1)=X((-1+r)+s)=X(-1+r) \cdot X(s)=(X(-1) \cdot X(r)) \cdot X(s)$. Since $X(-1), X(r) \cdot X(s) \in \mathcal{U}_{2}$, we have $(X(-1), X(r) \cdot X(s)) \in \Omega$ and hence $X(r+s-1)=X(-1) \cdot(X(r) \cdot X(s))$. Hence $\bar{X}(r+s)=\bar{X}(r) \cdot \bar{X}(s)$. One can conclude that $\bar{X}(r+s)=\bar{X}(r) \cdot \bar{X}(s)$ for $r, s \in(-1-\epsilon, 0)$ from the previous case and the definition of $\bar{X}$. Now suppose $r, s \in(-1-\epsilon, 1+\epsilon)$ and that $r \cdot s<0$. We will only show the case that $r \in(1,1+\epsilon)$ and $s \in[-1,0)$, the other cases being similar. Write $r=1+\delta$ with $0<\delta<\epsilon$. Then $\bar{X}(r+s)=\bar{X}(1+\delta+s)=\bar{X}((1+s)+\delta)$. Since $1+s \in[0,1)$, we have $\bar{X}(r+s)=X(1+s) \cdot X(\delta)=(X(1) \cdot X(s)) \cdot X(\delta)=X(1) \cdot(X(s) \cdot X(\delta))$. But since elements of image $(X)$ commute, we have $X(s) \cdot X(\delta)=X(\delta) \cdot X(s)$ and thus $\bar{X}(r+s)=X(1) \cdot(X(\delta) \cdot X(s))=(X(1) \cdot X(\delta)) \cdot X(s)=\bar{X}(r) \cdot \bar{X}(s)$.

\section{Consequences of NSS}

In this section we assume $G$ is NSS.

\section{Special Neighborhoods}

The next lemma is from [10, and we repeat its proof, with a more detailed justification of some steps.

Lemma 4.1. There is a neighborhood $V$ of 1 such that $V \subseteq \mathcal{U}_{2}$ and for all $x, y \in V$, if $x^{2}=y^{2}$, then $x=y$. 
Proof. Choose a compact symmetric neighborhood $U$ of 1 in $G$ with $U \subseteq \mathcal{U}_{6}$ such that $U$ contains no subgroups of $G$ other than $\{1\}$. Choose a symmetric open neighborhood $W$ of 1 in $G$ such that $W^{5} \subseteq U$. Since $U$ is compact, we can find a symmetric open neighborhood $V$ of 1 in $G$ such that $V^{2} \subseteq W$ and $g V g^{-1} \subseteq W$ for all $g \in U$. We claim that this $V$ fulfills the desired condition.

Suppose $x, y \in V$ are such that $x^{2}=y^{2}$. Let $a:=x^{-1} y \in V^{2} \subseteq W \subseteq U$. Since $V \subseteq \mathcal{U}_{5}$, we have

$$
\text { axa }=\left(x^{-1} y\right) x\left(x^{-1} y\right)=x^{-1} y^{2}=x^{-1} x^{2}=x .
$$

Claim: For all $n, a^{n}$ is defined, $a^{n} \in U$ and $a^{n}=x a^{-n} x^{-1}$.

We prove the claim by induction. The case $n=1$ follows from the fact that $a x a=x$. For the inductive step, suppose the assertion holds for all $m \in\{1, \ldots, n\}$. In order to show that $a^{n+1}$ is defined, it suffices to show that $\left(a^{i}, a^{j}\right) \in \Omega$ for all $i, j \in\{1, \ldots, n\}$ such that $i+j=n+1$. However, by the inductive hypothesis, $\left(a^{i}, a^{j}\right) \in U \times U \subseteq \Omega$, proving that $a^{n+1}$ is defined. Since $U \subseteq \mathcal{U}_{6}$, we have

$$
a^{n+1}=a^{n} \cdot a=\left(x a^{-n} x^{-1}\right)\left(x a^{-1} x^{-1}\right)=x a^{-n-1} x^{-1} .
$$

It remains to show that $a^{n+1} \in U$. First suppose that $n+1$ is even, say $n+1=2 m$. Then

$$
a^{n+1}=a^{m} \cdot a^{m}=x a^{-m} x^{-1} a^{m} \in x W \subseteq W^{2} \subseteq U .
$$

Now if $n+1$ is odd, then

$$
a^{n+1}=a^{n} \cdot a \in W^{2} \cdot W^{2} \subseteq U .
$$

This finishes the proof of the claim. The claim yields a subgroup $a^{\mathbb{Z}}$ of $G$ with $a^{\mathbb{Z}} \subseteq U$. Thus $a=1$ and so $x=y$.

Definition 4.2. A special neighborhood of $G$ is a compact symmetric neighborhood $\mathcal{U}$ of 1 in $G$ such that $\mathcal{U} \subseteq \mathcal{U}_{2}, \mathcal{U}$ contains no nontrivial subgroup of $G$, and for all $x, y \in \mathcal{U}$, if $x^{2}=y^{2}$, then $x=y$.

By the last lemma, we know that $G$ has a special neighborhood. In the rest of this section, we fix a special neighborhood $\mathcal{U}$ of $G$. Then every $a \in \boldsymbol{\mu} \backslash\{1\}$ is $\mathcal{U}$-pure. For $a \in G^{*}$, we set $\operatorname{ord}(a):=\operatorname{ord}_{\mathcal{U}}(a)$.

Lemma 4.3. Let $a \in G^{*}$. Then $\operatorname{ord}(a)$ is infinite iff $a \in \boldsymbol{\mu}$. 
Proof. We remarked earlier that if $a \in \boldsymbol{\mu}$, then $\operatorname{ord}(a)$ is infinite. Now suppose $\operatorname{ord}(a)$ is infinite. Then, for all $k \in \mathbb{Z}, a^{k}$ is defined and $a^{k} \in \mathcal{U}^{*}$. It is easy to prove by induction that $\operatorname{st}(a)^{k}$ is defined and $\operatorname{st}(a)^{k} \in \mathcal{U}$ for all $k \in \mathbb{Z}$. This implies that $\operatorname{st}(a)=1$ since $\mathcal{U}$ is a special neighborhood.

Lemma 4.4. Suppose $\sigma>\mathbb{N}$ and $a \in G(\sigma)$. Then:

(1) if $a \neq 1$, then $a$ is $\mathcal{U}$-pure and $\sigma=\mathrm{O}(\operatorname{ord}(a))$;

(2) if $i=\mathrm{o}(\sigma)$, then $a^{i}$ is defined and $a^{i} \in \boldsymbol{\mu}$;

(3) Let $\Sigma_{a}:=\Sigma_{\sigma, a, \mathcal{U}}$ and $r_{a}:=r_{\sigma, a, \mathcal{U}}$. Let $X_{a}:\left(-r_{a}, r_{a}\right) \rightarrow G$ be defined by $X(s):=\operatorname{st}\left(a^{[s \sigma]}\right)$. Then $X_{a}$ is a local 1-ps of $G$.

Proof. It has already been remarked why $a$ is $\mathcal{U}$-pure. If $\operatorname{ord}(a)=\mathrm{o}(\sigma)$, then $a^{\operatorname{ord}(a)} \in \boldsymbol{\mu}$, a contradiction. Hence, $\sigma=\mathrm{O}(\operatorname{ord}(a))$. By $(1)$, if $i=\mathrm{o}(\sigma)$, then $i=\mathrm{o}(\operatorname{ord}(a))$, whence $a^{i}$ is defined and $a^{i} \in \boldsymbol{\mu}$ since $a$ is $\mathcal{U}$-pure. This proves (2). (3) follows immediately from Lemma 3.14.

Corollary 4.5. Suppose $G$ is not discrete. Then $L(G) \neq\{\mathbb{O}\}$.

Proof. Take $a \in \boldsymbol{\mu} \backslash\{1\}$. Set $\sigma:=\operatorname{ord}(a)$. Since $a \in \boldsymbol{\mu}$, we must have $\sigma>\mathbb{N}$. Since $a \in G(\sigma)$, we have $\left[X_{a}\right] \in L(G)$, where $X_{a}$ is as defined in Lemma 4.4. We need to show that $\left[X_{a}\right] \neq \mathbb{O}$. It suffices to show that for every $n$ such that $\frac{1}{n} \in \Sigma_{a}, a^{\left[\frac{1}{n} \sigma\right]} \notin \boldsymbol{\mu}$. Let $t:=\left[\frac{1}{n} \sigma\right]$. Then $t=\frac{1}{n} \sigma-\epsilon$, with $\epsilon \in[0,1)^{*}$. Towards a contradiction, suppose that $a^{t} \in \boldsymbol{\mu}$. Then since $n t \leq \sigma, a^{n t}$ is defined and $a^{n t}=\left(a^{t}\right)^{n} \in \boldsymbol{\mu}$ by Lemma 2.18. Also $n \epsilon \in \mathbb{N}^{*}$ and $n \epsilon<n$, whence $a^{n \epsilon} \in \boldsymbol{\mu}$. But then $a^{\sigma}=a^{n t+n \epsilon}=a^{n t} \cdot a^{n \epsilon} \in \boldsymbol{\mu}$, a contradiction.

Lemma 4.6. Let $a \in G(\sigma) \backslash\{1\}$. Then:

(1) $a^{-1} \in G(\sigma)$ and $\left[X_{a^{-1}}\right]=(-1) \cdot\left[X_{a}\right]$;

(2) $b \in \boldsymbol{\mu} \Rightarrow b a b^{-1} \in G(\sigma)$ and $\left[X_{b a b^{-1}}\right]=\left[X_{a}\right]$;

(3) $\left[X_{a}\right]=\mathbb{O} \Leftrightarrow a \in G^{\mathrm{o}}(\sigma)$;

(4) $L(G)=\left\{\left[X_{a}\right] \mid a \in G(\sigma)\right\}$.

Proof. (1) is straightforward. As to (2), let $b \in \boldsymbol{\mu}$ and let $\tau:=\operatorname{ord}(a)$. We know that $\sigma=\mathrm{O}(\tau)$. One can show by internal induction on $\eta$ that $\left(b a b^{-1}\right)^{\eta}$ is defined and equal to $b a^{\eta} b^{-1}$ for all $\eta \leq \tau$. Thus, for $i=\mathrm{o}(\sigma)$, we have $\left(b a b^{-1}\right)^{i} \in \boldsymbol{\mu}$ and hence $b a b^{-1} \in G(\sigma)$. Again, for $r \in \Sigma_{a}$, we have that $\left(b a b^{-1}\right)^{[r \sigma]}$ is defined and equals $b a^{[r \sigma]} b^{-1}$, whence $\left[X_{a}\right]=\left[X_{b a b^{-1}}\right]$.

The proof of (3) is immediate from the definitions and Lemma 3.1. Now suppose $\mathbb{X} \in L(G)$ and $X \in \mathbb{X}$. Suppose domain $(X)=(-r, r)$ and consider the nonstandard extension $X:(-r, r)^{*} \rightarrow G^{*}$ of $X$. Let $a=X\left(\frac{1}{\sigma}\right) \in \boldsymbol{\mu}$. We claim that $\mathbb{X}=\left[X_{a}\right]$. To see this, let $s<\min \left(r, r_{a}\right)$ and let $\epsilon$ be an infinitesimal element of $\mathbb{R}^{*}$ such that $s=\frac{[s \sigma]}{\sigma}+\epsilon$. Then: 


$$
\begin{aligned}
X(s) & =\operatorname{st}\left(X\left(\frac{[s \sigma]}{\sigma}\right)\right) \\
& =\operatorname{st}\left(X\left(\frac{1}{\sigma}\right)^{[s \sigma]}\right) \\
& =\operatorname{st}\left(a^{[s \sigma]}\right) \\
& =X_{a}(s)
\end{aligned}
$$

Hence we have shown that $X$ and $X_{a}$ agree on $(-r, r) \cap\left(-r_{a}, r_{a}\right)$. This proves (4).

\section{The Local Exponential Map}

Consider the following sets:

$$
\mathcal{K}:=\{\mathbb{X} \in L(G) \mid I \subseteq \operatorname{domain}(\mathbb{X}) \text { and } \mathbb{X}(I) \subseteq \mathcal{U}\}
$$

and

$$
K:=\{\mathbb{X}(1) \mid \mathbb{X} \in \mathcal{K}\}
$$

Lemma 4.7. For every $\mathbb{X} \in L(G)$, there is $s \in(0,1)$ such that $s \cdot \mathbb{X} \in \mathcal{K}$.

Proof. Let $X \in \mathbb{X}$ and suppose $X:(-r, r) \rightarrow G$. If $r \leq 1$, pick any $0<s_{1}<r$ and then $s_{1} \cdot X:\left(-\frac{r}{s_{1}}, \frac{r}{s_{1}}\right) \rightarrow G$ will have $I$ contained in its domain. If $r>1$, let $s_{1}=1$. By continuity of $s_{1} \cdot X$, there is $0<s_{2}<1$ such that $\left(s_{2} \cdot\left(s_{1} \cdot X\right)\right)(I) \subseteq \mathcal{U}$. So for $s:=s_{1} \cdot s_{2}, s \cdot \mathbb{X} \in \mathcal{K}$.

Lemma 4.8. $K$ is closed in $\mathcal{U}$ and thus compact.

Proof. It suffices to show that if $c \in K^{*}$, then $\operatorname{st}(c) \in K$. Take an internal local 1-ps of $G^{*}$, say $Y:(-s, s) \rightarrow G^{*}$, such that $I^{*} \subset(-s, s), Y\left(I^{*}\right) \subseteq \mathcal{U}^{*}$, and with $Y(1)=c$. If $\delta$ is an infinitesimal element of $\mathbb{R}^{*}$, then $\operatorname{st}(Y(\mathbb{Z} \delta)) \subseteq \mathcal{U}$ is a subgroup of $G$, so $Y(\delta) \in \boldsymbol{\mu}$. Hence for each neighborhood $V$ of 1 in $G$ there is $n>0$ such that $\frac{1}{n}<s$ and $Y(r) \in V^{*}$ for all $r \in \mathbb{R}^{*}$ with $|r|<\frac{1}{n}$. If $\operatorname{st}(s)>1$, then there is $\epsilon \in \mathbb{R}^{>0}$ such that $1+\epsilon<s$. Let $X:(-1-\epsilon, 1+\epsilon) \rightarrow G$ be defined by $X(r)=\operatorname{st}(Y(r))$. Then $[X] \in \mathcal{K}$ and $\operatorname{st}(c)=[X](1) \in K$. Now suppose that $\operatorname{st}(s)=1$ and let $X: I \rightarrow G$ be defined by $X(r)=\operatorname{st}(Y(r))$. By Lemma 3.15. there is $\epsilon \in(0,1)$ and $\bar{X}:(-1-\epsilon, 1+\epsilon) \rightarrow G$ such that $\bar{X}$ is a local 1-ps of $G$ and $\bar{X} \mid I=X$. Then $[\bar{X}] \in \mathcal{K}$ and $\operatorname{st}(c)=[\bar{X}](1) \in K$. 
Lemma 4.9. The map $\mathbb{X} \rightarrow \mathbb{X}(1): \mathcal{K} \rightarrow K$ is bijective.

Proof. Let $\mathbb{X}_{1}, \mathbb{X}_{2}$ be in $\mathcal{K}$ with representatives $X_{1}, X_{2}$ containing $I$ in their domains. Suppose $X_{1}(1)=X_{2}(1)$. Then $\left(X_{1}\left(\frac{1}{2}\right)\right)^{2}=\left(X_{2}\left(\frac{1}{2}\right)\right)^{2}$, implying that $X_{1}\left(\frac{1}{2}\right)=X_{2}\left(\frac{1}{2}\right)$ since $\mathcal{U}$ is a special neighborhood. Inductively, we have $X_{1}\left(\frac{1}{2^{n}}\right)=X_{2}\left(\frac{1}{2^{n}}\right)$ for all $n$, and thus $X_{1}\left(\frac{k}{2^{n}}\right)=X_{2}\left(\frac{k}{2^{n}}\right)$ for all $k \in \mathbb{Z}$ such that $\left|\frac{k}{2^{n}}\right| \leq 1$. By density, we have $\left[X_{1}\right]=\left[X_{2}\right]$.

From now on, for $\mathbb{X} \in \mathcal{K}$, we let $E(\mathbb{X})=\mathbb{X}(1)$. So the last lemma now reads $E: \mathcal{K} \rightarrow K$ is a bijection. We will have much more to say about this local exponential map later and it will be a crucial tool in our solution of the Local H5.

\section{A Countable Neighborhood Basis of the Identity}

For $Q$ as earlier, we put $\operatorname{ord}(Q):=\operatorname{ord}_{\mathcal{U}}(Q)$. Extend this to the standard setting as follows: for symmetric $P \subseteq G$ with $1 \in P$, let $\operatorname{ord}(P)$ be the largest $n$ such that $P^{n}$ is defined and $P^{n} \subseteq \mathcal{U}$ if there is such an $n$ and set $\operatorname{ord}(P):=\infty$ if $P^{n}$ is defined and $P^{n} \subseteq \mathcal{U}$ for all $n$.

We set $V_{n}:=\{x \in G \mid \operatorname{ord}(x) \geq n\}$. Notice that for all $n$,

$$
p_{1}^{-1}(\mathcal{U}) \cap \ldots \cap p_{n}^{-1}(\mathcal{U}) \subseteq V_{n} \subseteq \mathcal{U}_{n} .
$$

Lemma 4.10. $\left(V_{n}: n \geq 1\right)$ is a decreasing sequence of compact symmetric neighborhoods of 1 in $G$, ord $\left(V_{n}\right) \rightarrow \infty$ as $n \rightarrow \infty$, and $\left\{V_{n}: n \geq 1\right\}$ is a countable neighborhood basis of 1 in $G$.

Proof. For $\sigma>\mathbb{N}$, consider the internal set

$$
V_{\sigma}:=\left\{g \in G^{*} \mid \operatorname{ord}(g) \geq \sigma\right\} .
$$

Note that $V_{\sigma} \subseteq \boldsymbol{\mu}$, so given any neighborhood $U$ of 1 in $G$, we have $x_{1} \cdots x_{m}$ is defined and in $U^{*}$ for all $m$ and $x_{1}, \ldots, x_{m} \in V_{m}^{*}$. It follows that for any neighborhood $U$ of 1 in $G$ and any $m$ we have $\left(V_{n}\right)^{m}$ is defined and contained in $U$ for all sufficiently large $n$.

\section{Local Gleason-Yamabe Lemmas}

The trickiest and most technical part of the original solution of Hilbert's fifth problem concerned several ingenious lemmas proved by Gleason and Yamabe. These lemmas really drive much of the proof and obtaining local versions of them is a crucial part of our current project. Fortunately, it is not too difficult to obtain local versions of these lemmas, although carrying out some of the details can become laborious. 
Throughout this section, we work with the following assumptions. We suppose $\mathcal{U} \subseteq \mathcal{W}$ are compact symmetric neighborhoods of 1 such that $\mathcal{W} \subseteq \mathcal{U}_{M}$ and $\mathcal{U}^{P} \subseteq \mathcal{W}$ for fixed positive integers $P \leq M$ large enough for all of the arguments below to make sense. (A careful analysis of the arguments below could give precise values for $M$ and $P$, but this endeavor became quite tedious.) We let $Q \subseteq \mathcal{U}$ be symmetric such that $N:=\operatorname{ord}_{\mathcal{U}}(Q) \neq \infty$ and $Q^{N+1}$ is defined. We first define the map $\Delta=\Delta_{Q}: G \rightarrow[0,1]$ by

- $\Delta(1)=0$

- $\Delta(x)=\frac{i}{N+1}$ if $x \in Q^{i} \backslash Q^{i-1}, 1 \leq i \leq N$;

- $\Delta(x)=1$ if $x \notin Q^{N}$.

Then for all $x \in G$,

- $\Delta(x)=1$ if $x \notin \mathcal{U}$;

- $|\Delta(a x)-\Delta(x)| \leq \frac{1}{N}$ for all $a \in Q$ such that $(a, x) \in \Omega$.

To smooth out $\Delta$, fix a continuous function $\tau: G \rightarrow[0,1]$ such that

$$
\tau(1)=1, \quad \tau(x)=0 \text { for all } x \in G \backslash \mathcal{U} .
$$

It is important later that $\tau$ depends only on $\mathcal{U}$, not on $Q$.

Next define $\theta=\theta_{Q}: G \rightarrow[0,1]$ by setting

$$
\theta(x)= \begin{cases}\sup _{y \in \mathcal{U}}(1-\Delta(y)) \tau\left(y^{-1} x\right) & \text { if } x \in \mathcal{W} \\ 0 & \text { if } x \in G \backslash \mathcal{W}\end{cases}
$$

Lemma 5.1. The following properties hold for the above defined functions.

(1) $\theta$ is continuous and $\theta(x)=0$ if $x \notin \mathcal{U}^{2}$;

(2) $0 \leq \tau \leq \theta \leq 1$

(3) $|\theta(a x)-\theta(x)| \leq \frac{1}{N}$ for $a \in Q$ if $(a, x) \in \Omega$.

Proof. It is clear that $\theta(x)=0$ if $x \notin \mathcal{U}^{2}$. To see that $\theta$ is continuous, let $x \in G$ and $a \in \boldsymbol{\mu}$. We must show that $\theta(a x)-\theta(x)$ is infinitesimal. First note that if $x \notin \mathcal{W}$, then $a x \notin \mathcal{W}^{*}$; in this case, $\theta(a x)=\theta(x)=0$. So assume $x \in \mathcal{W}$. If $a x \notin \mathcal{W}$, then $x \notin \operatorname{interior}(\mathcal{W})$. Since $\mathcal{U}^{2} \subseteq \operatorname{interior}(\mathcal{W})$, we conclude that $x \notin \mathcal{U}^{2}$ and so $\theta(x)=\theta(a x)=0$. Now suppose $a x \in \mathcal{W}^{*}$, so we can use the first clause in the definition of $\theta$ for $\theta(x)$ and $\theta(a x)$. In this case, $\theta(a x)-\theta(x)$ is infinitesimal by the continuity of $\tau$. (2) is also clear from the definition.

We now prove (3). Let $a \in Q$ and suppose $(a, x) \in \Omega$. If $x \notin \mathcal{W}$, then ax $\notin \mathcal{U}^{2}$, else $x \in \mathcal{U}^{3} \subseteq \mathcal{W}$, a contradiction. Hence $\theta(a x)=\theta(x)=0$ in this case. Now if $x \in \mathcal{W} \backslash \mathcal{U}^{3}$, then $a x \notin \mathcal{U}^{2}$, and once again $\theta(a x)=\theta(x)=0$. Hence we can suppose $x \in \mathcal{U}^{3}$ so that $a x \in \mathcal{U}^{4} \subseteq \mathcal{W}$ and so we can use 
the first clause in the definition of $\theta$ for $\theta(x)$ and $\theta(a x)$. Let $y \in \mathcal{U}$. Then $\left(a^{-1}, y\right) \in \Omega$ and

$$
\left|\left(1-\Delta\left(a^{-1} y\right)\right)-(1-\Delta(y))\right| \leq \frac{1}{N}
$$

Since $y^{-1} a x$ is defined, we have $y^{-1} a x=\left(a^{-1} y\right)^{-1} x$, so

$$
\left|(1-\Delta(y)) \tau\left(y^{-1} a x\right)-\left(1-\Delta\left(a^{-1} y\right) \tau\left(\left(a^{-1} y\right)^{-1} x\right)\right)\right| \leq \frac{1}{N} .
$$

(3) now follows from noting that the sets $\left\{(1-\Delta(y)) \tau\left(y^{-1} x\right) \mid y \in \mathcal{U}\right\}$ and $\left\{\left(1-\Delta\left(a^{-1} y\right)\right) \tau\left(\left(a^{-1} y\right)^{-1} x\right) \mid y \in \mathcal{U}, a^{-1} y \in \mathcal{U}\right\}$ have the same supremum.

Let $C:=\left\{f: G \rightarrow \mathbb{R} \mid f\right.$ is continuous and $\left.\operatorname{supp}(f) \subseteq \mathcal{W}^{2}\right\}$. Then $C$ is a real vector space and we equip it with the norm given by

$$
\|f\|:=\sup \{|f(x)| \mid x \in G\} .
$$

If $f \in C$ is such that $\operatorname{supp}(f) \subseteq \mathcal{W}$ and $a \in \mathcal{W}$, then we can define the new function $a \cdot f$ by the rule

$$
(a \cdot f)(x)= \begin{cases}f\left(a^{-1} x\right) & \text { if } x \in \mathcal{W}^{2} \\ 0 & \text { otherwise }\end{cases}
$$

It is easy to see that $a \cdot f \in C$ and that if $f, g \in C$ both have supports contained in $\mathcal{W}$, then $a \cdot(f+g)=a \cdot f+a \cdot g$. We will often drop the and just write $a f$ instead of $a \cdot f$. This should not be confused with the function $r f$ given by $(r f)(x)=r f(x)$ for $r \in \mathbb{R}$, which is defined for any $f: G \rightarrow \mathbb{R}$.

Lemma 5.2. Suppose $a, b \in \mathcal{W}$ and $f \in C$ is such that $\operatorname{supp}(f) \subseteq \mathcal{W}$ and $\operatorname{supp}(b f) \subseteq \mathcal{W}$. Then:

(i) $\|a f\|=\|f\|$;

(ii) $a(b f)=(a b) f$ and

$$
\|(a b) f-f\| \leq\|a f-f\|+\|b f-f\| .
$$

Proof. To see (i), note that

$$
\begin{aligned}
\|a f\| & =\sup \left\{\left|f\left(a^{-1} x\right)\right| \mid x \in \mathcal{W}^{2}\right\} \\
& =\sup \left\{\left|f\left(a^{-1} x\right)\right| \mid x \in \mathcal{W}^{2}, a^{-1} x \in \mathcal{W}\right\} \\
& =\sup \{|f(y)| \mid y \in \mathcal{W}\} \\
& =\|f\|
\end{aligned}
$$

We now prove (ii). Suppose $x \notin \mathcal{W}^{2}$. In this case, $((a b) f)(x)=0$ and since $a^{-1} x \notin \mathcal{W}$ and $\operatorname{supp}(b f) \subseteq \mathcal{W}$, we also have $(a(b f))(x)=0$. Now suppose $x \in \mathcal{W}^{2}$. Then $((a b) f)(x)=f\left((a b)^{-1} x\right)=f\left(\left(b^{-1} a^{-1}\right) x\right)$ and $(a(b f))(x)=(b f)\left(a^{-1} x\right)$. If $a^{-1} x \notin \mathcal{W}^{2}$, then $b^{-1} a^{-1} x \notin \mathcal{W}$; in this 
case, we have $((a b) f)(x)=0$ and $\left(a(b f)(x)=0\right.$. If $a^{-1} x \in \mathcal{W}^{2}$, then $(a(b f))(x)=(b f)\left(a^{-1} x\right)=f\left(b^{-1}\left(a^{-1} x\right)\right)=((a b) f)(x)$. We now finish the proof of (ii) as in the global case, that is

$$
\begin{aligned}
\|(a b) f-f\| & =\|a(b f)-a f+a f-f\| \\
& \leq\|a(b f)-a f\|+\|a f-f\| \\
& \leq\|a(b f-f)\|+\|a f-f\| \\
& =\|b f-f\|+\|a f-f\|
\end{aligned}
$$

Since $\operatorname{supp}(\theta) \subseteq \mathcal{U}^{2} \subset \mathcal{W}$, we can consider the function $a \theta \in C$ for any $a \in \mathcal{W}$. We will often need the following equicontinuity result.

(4) For each $\epsilon \in \mathbb{R}^{>0}$, there is a symmetric neighborhood $V_{\epsilon}$ of 1 in $G$, independent of $Q$, such that $V_{\epsilon} \subseteq \mathcal{U}$ and $\|a \theta-\theta\| \leq \epsilon$ for all $a \in V_{\epsilon}$. To see this, let $\epsilon \in \mathbb{R}^{>0}$ and note by the uniform continuity of $\tau$, we have a neighborhood $U$ of 1 in $G$ such that $|\tau(g)-\tau(h)|<\epsilon$ for all $g, h \in \mathcal{U}$ such that $g h^{-1} \in U$. Now take a symmetric neighborhood $V_{\epsilon}$ of 1 in $G$ such that $V_{\epsilon} \subseteq \mathcal{U}$ and $y^{-1} a y \in U$ for all $(a, y) \in \mathcal{U} \times V_{\epsilon}$. The claim is that this choice for $V_{\epsilon}$ works. Fix $a \in V_{\epsilon}$. We must compute $|(a \theta)(x)-\theta(x)|$ for various $x$. Let us first take care of some of the trivial computations. If $x \notin \mathcal{W}^{2}$, then $(a \theta)(x)=\theta(x)=0$. Next suppose that $x \in \mathcal{W}^{2} \backslash \mathcal{W}$, so $\theta(x)=0$. If $a^{-1} x \notin \mathcal{W}$, then $(a \theta)(x)=0$. If $a^{-1} x \in \mathcal{W}$, then $a^{-1} x \notin \mathcal{U}^{2}$, else $x \in \mathcal{U}^{3} \subseteq \mathcal{W}$, a contradiction. So in this case also, $(a \theta)(x)=0$. Now if $x \in \mathcal{W} \backslash \mathcal{U}^{3}$, then $a^{-1} x \notin \mathcal{U}^{2}$, so once again $(a \theta)(x)=\theta(x)=0$. Thus the only real case of interest is when $x \in \mathcal{U}^{3}$ and hence $a^{-1} x \in \mathcal{U}^{4} \subseteq \mathcal{W}$. In this case, we have $\left|\tau\left(y^{-1} a^{-1} x\right)-\tau\left(y^{-1} x\right)\right|<\epsilon$ for any $y \in \mathcal{U}$. We now have

$$
\begin{aligned}
|(a \theta)(x)-\theta(x)| & =\left|\sup _{y \in \mathcal{U}}(1-\Delta(y)) \tau\left(y^{-1} a^{-1} x\right)-\sup _{y \in \mathcal{U}}(1-\Delta(y)) \tau\left(y^{-1} x\right)\right| \\
& \leq \sup _{y \in \mathcal{U}}(1-\Delta(y))\left|\tau\left(y^{-1} a^{-1} x\right)-\tau\left(y^{-1} x\right)\right| \\
& \leq \epsilon .
\end{aligned}
$$

This finishes the proof of (4).

In [12], Montgomery and Zippin construct a linear functional

$$
f \mapsto \int f: C \rightarrow \mathbb{R}
$$

with the following properties: 
(i) For any $f \in C$, one has $\left|\int f\right| \leq \int|f|$;

(ii) (Left invariance) Suppose $f \in C$ satisfies $\operatorname{supp}(f) \subseteq \mathcal{W}$. Let $a \in \mathcal{W}$. Then $\int a f=\int f$.

The above defined functional will be referred to as the local Haar integral on $G$. As is the case for topological groups, the local Haar integral is unique up to multiplication by a positive real constant. By the Riesz Representation Theorem, for each local Haar integral as above, we get a Borel measure $\mu$ on a certain $\sigma$-algebra of subsets of $\mathcal{W}^{2}$. We will refer to the measure corresponding to a local Haar integral as a local Haar measure on $G$. If a given integral induces the measure $\mu$, then we will denote the integral of a function $f$ by $\int f d \mu$, or if we wish to specify the variable, by $\int f(x) d \mu(x)$. By the left invariance of the integral, the measure $\mu$ is left-invariant in the sense that for any measurable subset $V \subseteq \mathcal{W}$ and any $a \in \mathcal{W}$, we have $\mu(a V)=\mu(V)$.

With these remarks behind us, we can now proceed with our discussion. Fix a continuous function $\tau_{1}: G \rightarrow[0,1]$ such that

$$
\tau_{1}(x)=1 \text { for all } x \in \mathcal{U}^{2}, \quad \tau_{1}(x)=0 \text { for all } x \in G \backslash \mathcal{U}^{3} .
$$

It is important later that $\tau_{1}$ depends only on $\mathcal{U}$, not on $Q$.

Note that $0 \leq \theta \leq \tau_{1}$. Take the unique local Haar measure $\mu$ such that $\int \tau_{1}(x) d \mu(x)=1$. Then in particular we have

(5) $0 \leq \int \theta(x) d \mu(x) \leq 1$.

By (4) above, we have an open neighborhood $V \subseteq \mathcal{U}$ of 1 in $G$, independent of $Q$, such that $\theta^{2}(x) \geq \frac{1}{2}$ on $V$. We now introduce the function

$$
\phi=\phi_{Q}: G \rightarrow \mathbb{R}
$$

defined by

$$
\phi(x)= \begin{cases}\int \theta(x u) \theta(u) d \mu(u) & \text { if } x \in \mathcal{W} \\ 0 & \text { if } x \in G \backslash \mathcal{W}\end{cases}
$$

It should be clear that $\phi$ is continuous. The following are other useful properties of $\phi$ :

(6) $\operatorname{supp}(\phi) \subseteq \mathcal{U}^{4} \subseteq \mathcal{W}$;

(7) $\phi(1) \geq \frac{\mu(V)}{2}$;

(8) if $a \in Q$, then $\|a \phi-\phi\| \leq \frac{1}{N}$;

(9) $\|a \phi-\phi\| \leq\|a \theta-\theta\|$ for all $a \in \mathcal{W}$.

In verifying properties (8) and (9), one needs to perform the case distinctions that we have done earlier. As before, though, there is only one case where the first clause of the definition applies for both $a \phi$ and $\phi$ and in this case the results easily follow from the properties of the local Haar integral and 
the earlier derived properties (1)-(5). We use that $\mathcal{U}^{6} \subseteq \mathcal{W}$ in showing that there is only one genuine calculation to perform.

We should also point out that, by $(6), \operatorname{supp}(a \phi-\phi) \subseteq \mathcal{U}^{5} \subseteq \mathcal{W}$ for all $a \in \mathcal{W}$, and so for all $b \in \mathcal{W}$, we can consider the function $b(a \phi-\phi)$. We are finally in the position to prove the local versions of the Gleason-Yamabe Lemmas.

Lemma 5.3. Let $c, \epsilon \in \mathbb{R}^{>0}$. Then there is a neighborhood $U=U_{c, \epsilon} \subseteq \mathcal{U}$ of 1 in $G$, independent of $Q$, such that for all $a \in Q, b \in U$, and $m \leq c N$,

$$
\|b \cdot m(a \phi-\phi)-m(a \phi-\phi)\| \leq \epsilon, \quad\|m(a \phi-\phi)\| \leq c .
$$

Proof. Let $a \in Q, b \in \mathcal{U}$. Note that $\operatorname{supp}(b \cdot m(a \phi-\phi)-m(a \phi-\phi)) \subseteq \mathcal{U}^{6}$. So let $x \in \mathcal{U}^{6}$ and set $y:=b^{-1} x$. Then

$$
\begin{aligned}
(a \phi-\phi)(x) & =\int\left[\theta\left(a^{-1} x u\right)-\theta(x u)\right] \theta(u) d \mu(u) \\
b(a \phi-\phi)(x)=(a \phi-\phi)(y) & =\int\left[\theta\left(a^{-1} y u\right)-\theta(y u)\right] \theta(u) d \mu(u) .
\end{aligned}
$$

Assuming $P$ is large enough so that $y^{-1} x \in \mathcal{W}$, we can use left-invariance of the integral to replace $u$ by $x^{-1} y u$ in the function of $u$ integrated in the first identity. So,

$$
(a \phi-\phi)(x)=\int\left[\theta\left(a^{-1} y u\right)-\theta(y u)\right] \theta\left(x^{-1} y u\right) d \mu(u)
$$

Taking differences gives

$$
[b \cdot(a \phi-\phi)-(a \phi-\phi)](x)=\int[(a \theta-\theta)(y u)]\left[\left(\theta-y^{-1} x \theta\right)(u)\right] d \mu(u) .
$$

By (4), we can take the neighborhood $U_{c, \epsilon} \subseteq \mathcal{U}$ of 1 in $G$ so small that for all $b \in U_{c, \epsilon}$ and $x \in \mathcal{U}^{6}$ we have $y^{-1} x \in \mathcal{U}$ and

$$
\left\|\theta-y^{-1} x \theta\right\|<\frac{\epsilon}{c \mu\left(\mathcal{U}^{3}\right)} .
$$

It is straightforward to see that this choice of $U_{c, \epsilon}$ works.

Lemma 5.4. With $c, \epsilon \in \mathbb{R}^{>0}$, let $U=U_{c, \epsilon}$ be as in the previous lemma and let $a \in Q$ and $m, n$ be such that $m \leq c N, n>0, a^{n}$ is defined, and $a^{i} \in U$ for $i \in\{0, \ldots, n\}$. Then

$$
\left\|\left(\frac{m}{n}\right)\left(a^{n} \phi-\phi\right)-m(a \phi-\phi)\right\| \leq \epsilon .
$$

Proof. It is routine to check that $m\left(a^{n} \phi-\phi\right)=\sum_{i=0}^{n-1} a^{i} m(a \phi-\phi)$. Hence we have

$$
m\left(a^{n} \phi-\phi\right)-n m(a \phi-\phi)=\sum_{i=0}^{n-1}\left(a^{i} m(a \phi-\phi)-m(a \phi-\phi)\right) .
$$


By the previous lemma, we have for $i=0, \ldots, n-1$,

$$
\left\|a^{i} m(a \phi-\phi)-m(a \phi-\phi)\right\| \leq \epsilon
$$

which gives the desired result by summing and dividing by $n$.

Now suppose that $Q$ is a symmetric internal subset of $\boldsymbol{\mu}$ with $1 \in Q$ such that $N:=\operatorname{ord}_{\mathcal{U}}(Q) \in \mathbb{N}^{*}$. Note that $N>\mathbb{N}$. Then everything we have done so far in this section transfers to the nonstandard setting and yields internally continuous functions $\theta: G^{*} \rightarrow[0,1]^{*}$ and $\phi: G^{*} \rightarrow \mathbb{R}^{*}$ satisfying the internal versions of (1)-(9) and Lemmas 5.3 and 5.4. We now have the following lemma.

Lemma 5.5. Suppose $a \in Q, \nu=\mathrm{O}(N), a^{\nu}$ is defined and $a^{\sigma} \in \boldsymbol{\mu}$ for all $\sigma \leq \nu$. Then $\|\nu(a \phi-\phi)\|$ is infinitesimal.

Proof. Let $\epsilon \in \mathbb{R}^{>0}$. Choose $c \in \mathbb{R}^{>0}$ such that $\nu \leq c N$. Then by Lemma 5.4, with $m=n=\nu$, we have

$$
\left\|\left(a^{\nu} \phi-\phi\right)-\nu(a \phi-\phi)\right\| \leq \epsilon .
$$

Also $\left\|a^{\nu} \phi-\phi\right\| \leq\left\|a^{\nu} \theta-\theta\right\|<\epsilon$ by (9) and (4), so $\|\nu(a \phi-\phi)\| \leq 2 \epsilon$.

\section{Consequences of the Gleason-Yamabe Lemmas}

In this section, we bear the fruits of our labors from the previous section. We derive some consequences of the Gleason-Yamabe lemmas which allow us to put a group structure on $L(G)$. From this group structure on $L(G)$, we obtain the important corollary that in an NSS local group, the image of the local exponential map is a neighborhood of 1 , which later will allow us to conclude that NSS local groups are locally euclidean.

Throughout this section, we assume that $G$ is pure.

\section{Group structure on $L(G)$}

Lemma 6.1. Suppose $\nu>\mathbb{N}$ and $a_{1}, \ldots, a_{\nu}$ is an internal sequence such that $a_{i} \in G^{\mathrm{o}}(\nu)$ for all $i \in\{1, \ldots, \nu\}$. Then $a_{1} \cdots a_{\nu}$ is defined and $a_{1} \cdots a_{\nu} \in \boldsymbol{\mu}$.

Proof. Assume for the moment that $a_{1} \cdots a_{\nu}$ is defined. We shall prove that $a_{1} \cdots a_{\nu} \in \boldsymbol{\mu}$. Let $Q:=\left\{a_{1}, \ldots, a_{\nu}, a_{1}^{-1}, \ldots, a_{\nu}^{-1}\right\}$. Suppose, towards a contradiction, that $a:=a_{1} \cdots a_{\nu} \notin \boldsymbol{\mu}$. Then $Q$ is not degenerate. Since $G$ is pure, we have $\sigma>\mathbb{N}$ such that $Q^{\sigma}$ is defined, $Q^{\sigma} \nsubseteq \boldsymbol{\mu}$ but $Q^{i} \subseteq \boldsymbol{\mu}$ for all $i=$ $\mathrm{o}(\sigma)$. If $\nu>\sigma$, then we can replace $a_{1}, \cdots, a_{\nu}$ by a shorter internal sequence $b_{1}, \ldots, b_{\sigma}$ in $Q$ such that $b_{1} \cdots b_{\sigma} \notin \boldsymbol{\mu}$ and $Q$ by $\left\{1, b_{1}, \ldots, b_{\sigma}, b_{1}^{-1}, \ldots, b_{\sigma}^{-1}\right\}$. So we can suppose that $\nu \leq \sigma$ and in fact we can assume that $\sigma=\nu$ by changing the value of $\sigma$ if necessary. Choose $\mathcal{U}$ and $\mathcal{W}$ as in the previous 
section so that $\operatorname{st}(a) \notin \mathcal{U}^{4}$. Note that $\nu=\mathrm{O}\left(\operatorname{ord}_{\mathcal{U}}(Q)\right)$, else we would have that $Q^{\operatorname{ord} \mathcal{U}(Q)} \subseteq \boldsymbol{\mu}$, a contradiction. By transfer, the previous section yields the internally continuous function $\phi=\phi_{Q}: G^{*} \rightarrow \mathbb{R}^{*}$ satisfying the internal versions of all of the properties and lemmas from that section. In particular, $\phi\left(a^{-1}\right)=0$ and $\phi(1)$ is not infinitesimal. Hence $\|a \phi-\phi\|$ is not infinitesimal. However, by the transfer of Lemma 5.2, we have

$$
\|a \phi-\phi\| \leq \sum_{i=1}^{\nu}\left\|a_{i} \phi-\phi\right\|
$$

and the right hand side of the inequality is infinitesimal by Lemma 5.5, yielding a contradiction.

We will now prove that $a_{1} \cdots a_{\nu}$ is actually defined. To do this, we first fix an internal set $E \subseteq \boldsymbol{\mu}$.

Claim: If $b_{1}, \ldots, b_{\nu}$ is an internal sequence such that $b_{i}^{j}$ is defined and $b_{i}^{j} \in E$ for all $i, j \in\{1, \ldots, \nu\}$, then $b_{1} \cdots b_{\nu}$ is defined.

We prove this claim by internal induction on $\nu$. The claim is clearly true for $\nu=1$ and we now suppose inductively that it holds for a given $\nu$. Suppose $b_{1}, \ldots, b_{\nu+1}$ is an internal sequence such that $b_{i}^{j}$ is defined and $b_{i}^{j} \in E$ for all $i, j \in\{1, \ldots, \nu+1\}$. Then $b_{1} \cdots b_{\nu}$ is defined and $b_{i} \cdots b_{\nu+1}$ is defined for all $i \in\{2, \ldots, \nu+1\}$. By the first part of the proof of the lemma, we have $\left(b_{1} \cdots b_{i}, b_{i+1} \cdots b_{\nu+1}\right) \in \boldsymbol{\mu} \times \boldsymbol{\mu} \subseteq \Omega^{*}$ for all $i \in\{1, \ldots, \nu\}$, whence $b_{1} \cdots b_{\nu+1}$ is defined by Lemma 2.18. This finishes the proof of the claim.

Now given an internal sequence $a_{1}, \ldots, a_{\nu}$ as in the statement of the lemma, the claim implies that $a_{1} \cdots a_{\nu}$ is defined by taking $E$ to be the set of all $a_{i}^{j}$ for $i, j \in\{1, \ldots, \nu\}$.

Lemma 6.2. Suppose $U$ is a compact symmetric neighborhood of 1 in $G$ with $U \subseteq \mathcal{U}_{2}$. Let $\nu>\mathbb{N}$ be such that for all $i \in\{1, \ldots, \nu\}$, $a^{i}$ and $b^{i}$ are defined and $a^{i} \in U^{*}, b^{i} \in \boldsymbol{\mu}$. Then for all $i \in\{1, \ldots, \nu\}$, we have that $(a b)^{i}$ is defined and $(a b)^{i} \sim a^{i}$.

Proof. Suppose $i \in\{1, \ldots, \nu\}$. Then $\left(a^{i}, b\right) \in \Omega^{*}$ and $a^{i} b \sim a^{i}$. Since $\left(\operatorname{st}\left(a^{i}\right), \operatorname{st}\left(a^{-i}\right)\right) \in \Omega$, we have $\left(a^{i} b, a^{-i}\right) \in \Omega^{*}$. Similarly, $\left(a^{i}, b a^{-i}\right) \in \Omega^{*}$. Thus we can define the element $b_{i}:=a^{i} b a^{-i}$. For similar reasons, for any $i, j \in\{1, \ldots, \nu\}$, we can define the element $a^{i} b^{j} a^{-i}$. Note that $b_{i} \in \boldsymbol{\mu}$ for all $i \in\{1, \ldots, \nu\}$.

Claim 1: For all $i, j \in\{1, \ldots, \nu\}, b_{i}^{j}$ is defined and $b_{i}^{j}=a^{i} b^{j} a^{-i}$. 
We prove Claim 1 by internal induction on $j$. The case $j=1$ is obvious. Suppose the assertion is true for all $j^{\prime} \in\{1, \ldots, j\}$ and further suppose $j+1 \leq \nu$. We first show that $b_{i}^{j+1}$ is defined. Let $k, l \in\{1, \ldots, j\}$ be such that $k+l=j+1$. By the induction hypothesis, we know that $b_{i}^{k}$ is defined and $b_{i}^{k}=a^{i} b^{k} a^{-i}$. Since $b^{k} \in \boldsymbol{\mu}$, we have $b_{i}^{k} \in \boldsymbol{\mu}$. Similarly, we have $b_{i}^{l}$ is defined and $b_{i}^{l} \in \boldsymbol{\mu}$; thus $\left(b_{i}^{k}, b_{i}^{l}\right) \in \Omega^{*}$. So by Lemma 2.18, we have that $b_{i}^{j+1}$ is defined. But now, using that $b_{i}^{j} \in \boldsymbol{\mu}$, we have

$$
\begin{aligned}
b_{i}^{j+1} & =b_{i}^{j} \cdot b_{i} \\
& =\left(a^{i} b^{j} a^{-i}\right)\left(a^{i} b a^{-i}\right) \\
& =\left(\left(a^{i} b^{j} a^{-i}\right) \cdot\left(a^{i}\right)\right) \cdot\left(b a^{-i}\right) \\
& =\left(a^{i} b^{j}\right) \cdot\left(b a^{-i}\right) \\
& =\left(\left(a^{i} b^{j}\right) \cdot b\right) a^{-i} \\
& =a^{i} b^{j+1} a^{-i} .
\end{aligned}
$$

This proves Claim 1. Note that we have also shown that $b_{i}^{j} \in \boldsymbol{\mu}$ for all $i, j \in\{1, \ldots, \nu\}$. Then by Lemma 6.1, $b_{1} \cdots b_{i}$ is defined and $b_{1} \cdots b_{i} \in \boldsymbol{\mu}$ for all $i \in\{1, \ldots, \nu\}$.

The following claim finishes the proof of the lemma.

Claim 2: For all $i \in\{1, \ldots, \nu\},(a b)^{i}$ is defined and $(a b)^{i}=\left(b_{1} \cdots b_{i}\right) a^{i}$.

We prove Claim 2 by internal induction on $i$. The assertion is clearly true for $i=1$ and now suppose it holds for all $j \in \mathbb{N}^{*}$ with $j \leq i$. Suppose $i+1 \leq \nu$. To prove that $(a b)^{i+1}$ is defined, it is enough to check that $\left((a b)^{k},(a b)^{l}\right) \in \Omega^{*}$ for all $k, l \in\{1, \ldots, i\}$ with $k+l=i+1$. By the induction hypothesis, $(a b)^{k}=\left(b_{1} \cdots b_{k}\right) a^{k} \sim a^{k}$ and $(a b)^{l}=\left(b_{1} \cdots b_{l}\right) a^{l} \sim a^{l}$ and the desired result now follows from the fact that $\left(a^{k}, a^{l}\right) \in U^{*} \times U^{*}$. Next note that $a^{i+1} b=\left(a^{i+1} \cdot b\right) \cdot\left(a^{-i-1} a^{i+1}\right)=\left(a^{i+1} b a^{-i-1}\right) a^{i+1}=b_{i+1} a^{i+1}$. Hence, 
by the induction hypothesis, we have

$$
\begin{aligned}
(a b)^{i+1} & =\left((a b)^{i}\right) \cdot(a b) \\
& =\left(\left(b_{1} \cdots b_{i}\right) a^{i}\right) \cdot(a b) \\
& =\left(b_{1} \cdots b_{i}\right) \cdot\left(a^{i}(a b)\right) \\
& =\left(b_{1} \cdots b_{i}\right)\left(a^{i+1} b\right) \\
& =\left(b_{1} \cdots b_{i}\right)\left(b_{i+1} a^{i+1}\right) \\
& =\left(\left(b_{1} \cdots b_{i}\right) b_{i+1}\right) a^{i+1} \\
& =\left(b_{1} \cdots b_{i+1}\right) a^{i+1} .
\end{aligned}
$$

Lemma 6.3. Let $\nu>\mathbb{N}$ and $a \in G(\nu)$ be such that $a^{\nu}$ is defined and $a^{i} \in G_{\mathrm{ns}}^{*}$ for all $i \in\{1, \ldots, \nu\}$. Suppose also that $b \in \boldsymbol{\mu}$ is such that $b^{\nu}$ is defined and $b^{i} \in G_{\mathrm{ns}}^{*}$ and $a^{i} \sim b^{i}$ for all $i \in\{1, \ldots, \nu\}$. Then $a^{-1} b \in G^{\mathrm{o}}(\nu)$.

Proof. We first make a series of reductions. Note that by Lemma 6.2, if $a, b \in G(\nu)$, then $a^{-1} b \in G(\nu)$. Let $V$ be a compact symmetric neighborhood of 1 in $G$ such that $V \subseteq \mathcal{U}_{2}$. Then since $\nu=\mathrm{O}\left(\operatorname{ord}_{V}\left(a^{-1} b\right)\right)$, by replacing $\nu$ with $\operatorname{ord}_{V}\left(a^{-1} b\right)$ if $\nu>\operatorname{ord}_{V}\left(a^{-1} b\right)$, we may as well assume that $\left(a^{-1} b\right)^{i}$ is defined and $\left(a^{-1} b\right)^{i} \in G_{\mathrm{ns}}^{*}$ for all $i \in\{1, \ldots, \nu\}$. Let $Q=\left\{1, a, a^{-1}, b, b^{-1}\right\}$. Let $\tau$ be the largest element of $\mathbb{N}^{*}$ such that $Q^{\tau}$ is defined. Suppose $\tau<\nu$. If $\tau=\mathrm{o}(\nu)$, then by Lemma 6.1, $Q^{i} \subseteq \boldsymbol{\mu}$ for all $i \in\{1, \ldots, \tau\}$. But then, by Lemma 2.18, $Q^{\tau+1}$ is defined, a contradiction. Thus $\nu=O(\tau)$ and so, by replacing $\nu$ by $\tau$ if necessary, we can assume $Q^{\nu}$ is defined. If $a \in G^{\mathrm{o}}(\nu)$, then by Lemma 6.2, $\left(a^{-1} b\right)^{i}$ is defined and $\left(a^{-1} b\right)^{i} \sim a^{-i} \in \boldsymbol{\mu}$ for all $i \in\{1, \ldots, \nu\}$, yielding the desired conclusion. So we may as well assume that $a \notin G^{\mathrm{o}}(\nu)$ and by replacing $\nu$ by a smaller element of its archimedean class, we may as well assume that $a^{\nu} \notin \boldsymbol{\mu}$.

Now suppose, towards a contradiction, that there is $j \in\{1, \ldots, \nu\}$ such that $\left(a^{-1} b\right)^{j} \notin \boldsymbol{\mu}$. Note we must have $\nu=\mathrm{O}(j)$. Choose compact symmetric neighborhoods $\mathcal{U}$ and $\mathcal{W}$ of 1 in $G$ as in the previous section so that

$$
a^{\nu} \in \mathcal{W}^{*} \backslash \mathcal{U}^{*} \text { and }\left(a^{-1} b\right)^{j} \in \mathcal{W}^{*} \backslash\left(\mathcal{U}^{*}\right)^{4} .
$$

Let $\sigma=\operatorname{ord}_{\mathcal{U}}(Q)$. Then $\nu=\mathrm{O}(\sigma)$ by Lemma 6.1 Let $\phi=\phi_{Q}: G^{*} \rightarrow \mathbb{R}^{*}$ be the internally continuous function constructed in the previous section. Then we know that $\phi\left(\left(a^{-1} b\right)^{j}\right)=0$ and $\epsilon:=\phi(1)>0$ and is not infinitesimal. Also, note 


$$
\begin{aligned}
\epsilon & \leq\left\|\left(b^{-1} a\right)^{j} \phi-\phi\right\| \\
& \leq j\left\|\left(b^{-1} a\right) \phi-\phi\right\| \\
& =j\|a \phi-b \phi\| \\
& =\|j(a \phi-\phi)-j(b \phi-\phi)\| .
\end{aligned}
$$

We will obtain a contradiction by showing that $\|j(a \phi-\phi)-j(b \phi-\phi)\|<\epsilon$. By Lemma 5.4, there is a compact symmetric neighborhood $U \subseteq \mathcal{U}$ of 1 in $G$ such that for all $k>0$ in $\mathbb{N}^{*}$, if $a^{i} \in U^{*}$ and $b^{i} \in U^{*}$ for all $i \in\{1, \ldots, k\}$, then

$$
\begin{gathered}
\left\|\left(\frac{j}{k}\right)\left(a^{k} \phi-\phi\right)-j(a \phi-\phi)\right\|<\frac{\epsilon}{3}, \\
\left\|\left(\frac{j}{k}\right)\left(b^{k} \phi-\phi\right)-j(b \phi-\phi)\right\|<\frac{\epsilon}{3} .
\end{gathered}
$$

Let $k=\min \left\{\operatorname{ord}_{U}(a), \operatorname{ord}_{U}(b)\right\}$. Then the above equalities hold for this $k$. Note that $\nu=\mathrm{O}(k)$ and hence $j=O(k)$. Since $k<\nu$, we have $a^{k} \sim b^{k}$ and so

$$
\left\|\left(\frac{j}{k}\right)\left(a^{k} \phi-\phi\right)-\left(\frac{j}{k}\right)\left(b^{k} \phi-\phi\right)\right\|=\frac{j}{k}\left\|a^{k} \phi-b^{k} \phi\right\| \sim 0 .
$$

Combining this fact with the previous inequalities yields

$$
\|j(a \phi-\phi)-j(b \phi-\phi)\|<\epsilon,
$$

giving the desired contradiction.

Theorem 6.4. Suppose $\sigma>\mathbb{N}$. Then

(1) $G(\sigma)$ and $G^{\circ}(\sigma)$ are normal subgroups of $\boldsymbol{\mu}$;

(2) if $a \in G(\sigma)$ and $b \in \boldsymbol{\mu}$, then $a b a^{-1} b^{-1} \in G^{\mathrm{o}}(\sigma)$;

(3) $G(\sigma) / G^{\mathrm{o}}(\sigma)$ is abelian.

Proof. We have already remarked that $G(\sigma)$ and $G^{\circ}(\sigma)$ are symmetric and that $G(\sigma)$ is closed under multiplication. That $G(\sigma)$ is a normal subgroup of $\boldsymbol{\mu}$ is part of the content of Lemma 4.6, (2). Lemma 6.2 implies that $G^{\mathrm{o}}(\sigma)$ is a group. Now suppose $a \in G^{\mathrm{o}}(\sigma)$ and $b \in \boldsymbol{\mu}$. We need $b a b^{-1} \in G^{\mathrm{o}}(\sigma)$. One can show by internal induction on $\eta \leq \sigma$ that $\left(b a b^{-1}\right)^{\eta}$ is defined and equal to $b a^{\eta} b^{-1}$. In particular, this shows that $b a b^{-1} \in G^{\mathrm{o}}(\sigma)$. This finishes the proof of (1).

Let $a \in G(\sigma)$ and $b \in \boldsymbol{\mu}$. We need to show that $a b a^{-1} b^{-1} \in G^{\mathrm{o}}(\sigma)$. Note that $b a^{-1} b^{-1} \in G(\sigma)$ by (1). Let $U \subseteq \mathcal{U}_{2}$ be a compact symmetric neighborhood of 1. Choose $\tau \in\{1, \ldots, \sigma\}$ such that $\sigma=\mathrm{O}(\tau), a^{\tau}$ and $\left(b a^{-1} b^{-1}\right)^{\tau}$ are defined, and $a^{i} \in U^{*}$ for $i \in\{1, \ldots, \tau\}$. One can prove by internal induction that for $i \in\{1, \ldots, \tau\},\left(b a^{-1} b^{-1}\right)^{i}=b a^{-i} b^{-1}$ and hence $a^{-i} \sim\left(b a^{-1} b^{-1}\right)^{i}$. 
By Lemma 6.3, $a b a^{-1} b^{-1} \in G^{\mathrm{o}}(\tau) \subseteq G^{\mathrm{o}}(\sigma)$. This finishes (2) and (2) obviously implies (3).

Observe that if $\mathbb{X} \in L(G)$ and $\sigma>\mathbb{N}$, then $\mathbb{X}\left(\frac{1}{\sigma}\right) \in G(\sigma)$. To see this, suppose that $i=\mathrm{o}(\sigma)$. Then $\left(\mathbb{X}\left(\frac{1}{\sigma}\right)\right)^{i}=\mathbb{X}\left(\frac{i}{\sigma}\right) \in \boldsymbol{\mu}$.

Theorem 6.5. The map $S: L(G) \rightarrow G(\sigma) / G^{\mathrm{o}}(\sigma)$ defined by

$$
S(\mathbb{X})=\mathbb{X}\left(\frac{1}{\sigma}\right) G^{\mathrm{o}}(\sigma)
$$

is a bijection.

Proof. Suppose $\mathbb{X}, \mathbb{Y} \in L(G)$ and $S(\mathbb{X})=S(\mathbb{Y})$. Letting $a:=\mathbb{X}\left(\frac{1}{\sigma}\right)$ and $b:=\mathbb{Y}\left(\frac{1}{\sigma}\right)$, we have that $a^{-1} b \in G^{\mathrm{o}}(\sigma)$. Let $U$ be a compact symmetric neighborhood of 1 in $G$ with $U \subseteq \mathcal{U}_{2}$. Let $\tau:=\min \left\{\operatorname{ord}_{U}(a), \sigma\right\}$. By Lemma 6.2, we have $\left(a \cdot\left(a^{-1} b\right)\right)^{i}$ is defined, nearstandard and infinitely close to $a^{i}$ for all $i \in\{1, \ldots, \tau\}$, i.e. $a^{i} \sim b^{i}$ for all $i \in\{1, \ldots, \tau\}$. Then for all $i \in\{1, \ldots, \tau\}$ such that $\frac{i}{\sigma} \in \operatorname{domain}(\mathbb{X}) \cap \operatorname{domain}(\mathbb{Y})$, we have $\mathbb{X}\left(\frac{i}{\sigma}\right)=\mathbb{Y}\left(\frac{i}{\sigma}\right)$. Since $\sigma=\mathrm{O}(\tau)$, this implies that $\mathbb{X}=\mathbb{Y}$. Hence $S$ is $1-1$.

Now suppose $b \in G(\sigma) \backslash G^{\mathrm{o}}(\sigma)$. Consider the local 1-ps $X_{b}$ of $G$ defined by $X_{b}(t)=\operatorname{st}\left(b^{[t \sigma]}\right)$ on its domain; see Lemma 3.14. Let $b_{1}=X_{b}\left(\frac{1}{\sigma}\right) \in \boldsymbol{\mu}$. Choose $\tau \in\{1, \ldots, \sigma\}$ such that $\sigma=\mathrm{O}(\tau)$ and $\frac{\tau}{\sigma} \in \operatorname{domain}\left(X_{b}\right)$. Note then that $b_{1}^{\tau}$ is defined. For any $i \in\{1, \ldots, \tau\}, b^{i}$ is defined and $b^{i} \sim b_{1}^{i}$. By Lemma 6.3, $b^{-1} b_{1} \in G^{\mathrm{o}}(\tau) \subseteq G^{\mathrm{o}}(\sigma)$ and hence $b G^{\mathrm{o}}(\sigma)=b_{1} G^{\mathrm{o}}(\sigma)$. Hence $S$ is onto.

We use Theorem 6.5 to equip $L(G)$ with an abelian group operation $+_{\sigma}$. More explicitly, for $\mathbb{X}, \mathbb{Y} \in L(G)$, we define $\mathbb{X}+{ }_{\sigma} \mathbb{Y}:=S^{-1}(S(\mathbb{X}) \cdot S(\mathbb{Y}))$. By the proof of Theorem 6.5. we see that if $t \in \operatorname{domain}(\mathbb{X}+\sigma \mathbb{Y})$ and $\nu$ is such that $\frac{\nu}{\sigma} \sim t$, then

$$
\left(\mathbb{X}+{ }_{\sigma} \mathbb{Y}\right)(t) \sim\left[\mathbb{X}\left(\frac{1}{\sigma}\right) \mathbb{Y}\left(\frac{1}{\sigma}\right)\right]^{\nu}
$$

In [6], a proof is given, in the global setting, that this group operation is independent of $\sigma$. A local version of that proof is probably possible, but this independence also follows from the Local H5, which we prove without using that $+_{\sigma}$ is independent of $\sigma$. In light of this independence of $\sigma$, we write $\mathbb{X}+\mathbb{Y}$ instead of $\mathbb{X}+{ }_{\sigma} \mathbb{Y}$.

\section{Existence of Square Roots}

In the rest of this section, we assume that $G$ is NSS and that $\mathcal{U}$ is a special neighborhood of 1 in $G$. We now lead up to the proof of the crucial fact that the image of the local exponential map is a neighborhood of $1 \mathrm{in} G$. In 
particular, we will see that there is an open neighborhood of 1 in $G$ which is ruled by local 1-parameter subgroups in the sense that every element in this open neighborhood lies on some local 1-ps of $G$. In the rest of this section, we do not follow [6], but rather [3], which contains the following series of lemmas in the global setting.

Lemma 6.6. Suppose $\sigma>\mathbb{N}$ and $a, b \in G(\sigma)$. Then $\left[X_{a}\right]+\left[X_{b}\right]=\left[X_{a b}\right]$.

Proof. From Theorem 6.5, we have

$$
\begin{aligned}
S\left(\left[X_{a}\right]+\left[X_{b}\right]\right) & =S\left(\left[X_{a}\right]\right) \cdot S\left(\left[X_{b}\right]\right) \\
& =\left(a G^{\mathrm{o}}(\sigma)\right)\left(b G^{\mathrm{o}}(\sigma)\right) \\
& =(a b) G^{\mathrm{o}}(\sigma) \\
& =S\left(\left[X_{a b}\right]\right) .
\end{aligned}
$$

The result now follows since $S$ is injective.

The global version of the next lemma is in Singer [17], but the proof there is unclear.

Lemma 6.7. Suppose $\sigma>\mathbb{N}$ and $a \in G(\sigma)$. Then $a=b c^{2}$ with $b \in G^{\mathrm{o}}(\sigma)$ and $c \in G(\sigma)$.

Proof. Let $c:=X_{a}\left(\frac{1}{2 \sigma}\right) \in \boldsymbol{\mu}$. Then $c^{-2}=X_{a}\left(-\frac{1}{\sigma}\right)=X_{a^{-1}}\left(\frac{1}{\sigma}\right)$, which implies that $\left[X_{c^{-2}}\right]=\left[X_{a^{-1}}\right]$. From this, we conclude that

$$
\left[X_{a c^{-2}}\right]=\left[X_{a}\right]+\left[X_{c^{-2}}\right]=\left[X_{1}\right]=\mathbb{O} \text {. }
$$

Let $b:=a c^{-2}$. Then since $\left[X_{b}\right]=\mathbb{O}$, we have $b \in G^{\mathrm{o}}(\sigma)$.

Lemma 6.8. There exists $q \in \mathbb{Q}^{>0}$ such that for all $a, b, c \in \boldsymbol{\mu}$, if $a=b c^{2}$ and $\operatorname{ord}(b) \geq \operatorname{ord}(a)$, then $\operatorname{ord}(c) \geq q \cdot \operatorname{ord}(a)$.

Proof. Suppose no such $q$ exists. Then for all $n \geq 1$, there are $a_{n}, b_{n}, c_{n} \in \boldsymbol{\mu}$ such that $a_{n}=b_{n} c_{n}^{2}$ with $\operatorname{ord}\left(b_{n}\right) \geq \operatorname{ord}\left(a_{n}\right)$ and $n \operatorname{ord}\left(c_{n}\right)<\operatorname{ord}\left(a_{n}\right)$. By saturation, we have $a, b, c \in \boldsymbol{\mu}$ such that $a=b c^{2}, \operatorname{ord}(b) \geq \operatorname{ord}(a)$ and $n \operatorname{ord}(c)<\operatorname{ord}(a)$ for all $n$. Let $\sigma:=\operatorname{ord}(c)>\mathbb{N}$. Then

$$
\mathbb{O}=\left[X_{a}\right]=\left[X_{b c^{2}}\right]=\left[X_{b}\right]+\left[X_{c^{2}}\right]=\mathbb{O}+2\left[X_{c}\right],
$$

implying that $\left[X_{c}\right]=\mathbb{O}$, an obvious contradiction.

Fix $q$ as in the above lemma. For $a, b \in \boldsymbol{\mu}$, we let $[a, b]:=a b a^{-1} b^{-1}$, the commutator of $a$ and $b$. We will frequently use the identity $a b=[a, b] b a$. 
Lemma 6.9. Let $a \in \boldsymbol{\mu}$. Then for all $\nu \geq 1$, there are $b_{\nu}, c_{\nu} \in G^{*}$ such that $b_{\nu} \cdot c_{\nu} \cdot c_{\nu}$ is defined, $a=b_{\nu} c_{\nu}^{2}, \operatorname{ord}\left(b_{\nu}\right) \geq \nu \operatorname{ord}(a)$ and $\operatorname{ord}\left(c_{\nu}\right) \geq q \operatorname{ord}(a)$.

Proof. Note that the assertion is trivial if $a=1$ and so we suppose $a \neq 1$. Next note that if the assertion is true for a given $\nu$, then we have $b_{\nu}, c_{\nu} \in \boldsymbol{\mu}$ as they will have infinite order. By Lemmas 6.7 and 6.8, the assertion is true for some $\nu>\mathbb{N}$. It is clearly then true for every $\nu^{\prime} \in\{1, \ldots, \nu\}$. Hence, if we can prove the assertion for $\nu+1$, then we will be done by internal induction. If $b_{\nu}=1$, then the assumed factorization witnesses the truth of the assertion for all $\nu^{\prime}$. We thus assume $b_{\nu} \neq 1$. By Lemma 6.7, there are $b, c \in \boldsymbol{\mu}$ and $\tau_{1}>\mathbb{N}$ such that $b_{\nu}=b c^{2}$ with $\operatorname{ord}(b) \geq \tau_{1} \operatorname{ord}\left(b_{\nu}\right)$. Note that then $\operatorname{ord}(b) \geq \tau_{1} \nu \operatorname{ord}(a)$. By Lemma 6.8, we also know that $\operatorname{ord}(c) \geq q \operatorname{ord}\left(b_{\nu}\right) \geq q \nu \operatorname{ord}(a)$. Since $\boldsymbol{\mu}$ is a group, we have

$$
\begin{aligned}
a=b c^{2} c_{\nu}^{2} & =b c c c_{\nu} c_{\nu} \\
& =b c\left[c, c_{\nu}\right] c_{\nu} c c_{\nu} \\
& =b\left[c,\left[c, c_{\nu}\right]\right]\left[c, c_{\nu}\right] c c_{\nu} c c_{\nu} \\
& =b\left[c,\left[c, c_{\nu}\right]\right]\left[c, c_{\nu}\right]\left(c c_{\nu}\right)^{2} \\
& =b_{\nu+1}\left(c_{\nu+1}\right)^{2},
\end{aligned}
$$

where $b_{\nu+1}:=b\left[c,\left[c, c_{\nu}\right]\right]\left[c, c_{\nu}\right]$ and $c_{\nu+1}:=c c_{\nu}$. Now note that there is $\tau_{2}>\mathbb{N}$ such that

$$
\operatorname{ord}\left(\left[c, c_{\nu}\right]\right) \geq \tau_{2} \operatorname{ord}(c) \geq \tau_{2} q \nu \operatorname{ord}(a) .
$$

Also there is $\tau_{3}>\mathbb{N}$ such that

$$
\operatorname{ord}\left(\left[c,\left[c, c_{\nu}\right]\right]\right) \geq \tau_{3} \tau_{2} \operatorname{ord}(c) \geq \tau_{3} \tau_{2} q \nu \operatorname{ord}(a) .
$$

In combination with the facts that $\operatorname{ord}(b) \geq \tau_{1} \nu \operatorname{ord}(a)$ and $G(\tau \operatorname{ord}(a))$ is a group, where $\tau:=\min \left(\tau_{1}, \tau_{2}\right)$, we have that $\operatorname{ord}\left(b_{\nu+1}\right) \geq(\nu+1) \operatorname{ord}(a)$. Finally, Lemma 6.8 yields that $\operatorname{ord}\left(c_{\nu+1}\right) \geq q \operatorname{ord}(a)$ and so we are finished.

Lemma 6.10. For every $a \in \boldsymbol{\mu}$, there is $b \in \boldsymbol{\mu}$ with $a=b^{2}$.

Proof. Fix $\nu>\mathbb{N}$. Then by the previous lemma, the internal set of all $\eta \in \mathbb{N}$ such that for each $x \in V_{\eta}$ there are $y, z \in \mathcal{U}^{*}$ such that $y \cdot z \cdot z$ is defined, $x=y z^{2}, \operatorname{ord}(y) \geq \nu \operatorname{ord}(x)$ and $\operatorname{ord}(z) \geq q \operatorname{ord}(x)$ includes all infinite $\eta$. By underspill, there is some $n>0$ in this set. Now let $x \in V_{n}$. Then there are $y, z \in \mathcal{U}^{*}$ such that $y \cdot z \cdot z$ is defined, $x=y z^{2}$ and $\operatorname{ord}(y) \geq \nu \operatorname{ord}(x)$. Since $\operatorname{ord}(y)$ is infinite, $y \in \boldsymbol{\mu}$. Hence $x=\operatorname{st}(z)^{2}$, i.e. every element of $V_{n}$ has a square root in $\mathcal{U}$. By transfer, this implies that every $a \in \boldsymbol{\mu}$ has a square root $b \in \mathcal{U}^{*}$ and $b \in \boldsymbol{\mu}$, else the group $\{1, \operatorname{st}(b)\}$ is contained in $\mathcal{U}$, a contradiction. 
Lemma 6.11. Suppose $a \in \boldsymbol{\mu}, b \in G^{*}$ and $\nu>0$ are such that $\operatorname{ord}(b) \geq \nu$ and $a=b^{\nu}$. Then for all $i \in\{1, \ldots, \nu\}$, we have $b^{i} \in \boldsymbol{\mu}$.

Proof. We begin the proof with a claim.

Claim: For all $n, b^{n \nu}$ is defined.

We prove this claim by induction on $n$, the case $n=1$ being true by assumption. Assume inductively that $b^{n \nu}$ is defined. We claim that

$$
b^{j} \in \mathcal{U}_{2}^{*} \text { for all } j \in\{1, \ldots, n \nu\} .
$$

To see this, write $j=n^{\prime} \nu+j^{\prime}$, where $n^{\prime} \in \mathbb{N}$ and $j^{\prime} \in\{1, \ldots, \nu-1\}$. Then

$$
b^{j}=\left(b^{\nu}\right)^{n^{\prime}} \cdot b^{j^{\prime}} \sim b^{j^{\prime}} \in \mathcal{U}^{*} .
$$

In order to show that $b^{n \nu+\nu}$ is defined, we prove by internal induction on $i$ that $b^{n \nu+i}$ is defined and $b^{n \nu+i} \in \mathcal{U}_{2}^{*}$ for all $i \in\{0, \ldots, \nu\}$. The case $i=0$ is true since $b^{n \nu}=a^{n} \in \boldsymbol{\mu}$. Suppose, inductively, that it holds for a given $i$ and that $i+1 \leq \nu$. Let $k, l \in\{1, \ldots, n \nu+i\}$ be such that $k+l=n \nu+i+1$. Then by $(\dagger)$ and the inductive assumption, $\left(b^{k}, b^{i}\right) \in \mathcal{U}_{2}^{*} \times \mathcal{U}_{2}^{*} \subseteq \Omega^{*}$. Hence $b^{n \nu+i+1}$ is defined. But then $b^{i+1} \in \mathcal{U}^{*}$ and $b^{n \nu+i+1} \sim b^{i+1}$. Hence $b^{n \nu+i+1} \in \mathcal{U}_{2}^{*}$. This proves the claim.

Now suppose, towards a contradiction, that there is $i \in\{1, \ldots, \nu-1\}$ such that $b^{i} \notin \boldsymbol{\mu}$. Let $x:=\operatorname{st}\left(b^{i}\right) \in \mathcal{U} \backslash\{1\}$. From the claim, we see that if $x^{n}$ is defined for a given $n$, then $x^{n} \in \mathcal{U}$. From this, one can show by induction that for all $n, x^{n}$ is defined and $x^{n} \in \mathcal{U}$. Hence $x^{\mathbb{Z}} \subseteq \mathcal{U}$, a contradiction.

Lemma 6.12. Given $a \in \boldsymbol{\mu}$ and $\nu$, there is $b \in \boldsymbol{\mu}$ such that $\operatorname{ord}(b) \geq 2^{\nu}$ and $a=b^{2^{\nu}}$.

Proof. We prove this by internal induction on $\nu$, noting that the assertion is internal since $b \in \boldsymbol{\mu}$ can be replaced by $b \in G^{*}$ by the previous lemma. The assertion is true for $\nu=1$ by Lemma 6.10. Now suppose the assertion is true for $\nu$, i.e. that $a=b^{2^{\nu}}$ with $\operatorname{ord}(b) \geq 2^{\nu}$. Since $b \in \boldsymbol{\mu}$, there is $c \in \boldsymbol{\mu}$ with $b=c^{2}$ by Lemma 6.10 .

Claim: For all $i \in\left\{1, \ldots, 2^{\nu}\right\}, c^{2 i}$ is defined and $\left(c^{2}\right)^{i}=c^{2 i}$.

We prove the claim by internal induction. It is obviously true for $i=1$ and suppose it is true for a given $i \leq 2^{\nu}$. Suppose $i+1 \leq 2^{\nu}$. We first show $c^{2 i+1}$ is defined. By induction, we know that $c^{2 i}$ is defined, and so by Lemma 2.18, it is enough to show $\left(c^{k}, c^{l}\right) \in \Omega^{*}$ for $k, l \in\{1, \ldots, 2 i\}$ with $k+l=2 i+1$. If $k$ is even, then

$$
c^{k}=\left(c^{2}\right)^{\frac{k}{2}}=b^{\frac{k}{2}} \in \boldsymbol{\mu}
$$


by Lemma 6.11 since $\frac{k}{2} \leq i \leq 2^{\nu}$. Similarly, if $k$ is odd, then

$$
c^{k}=c \cdot\left(c^{2}\right)^{\frac{k-1}{2}}=c b^{\frac{k-1}{2}} \in \boldsymbol{\mu} .
$$

Thus, for $k, l \in\{1, \ldots, 2 i\}$, one has $\left(c^{k}, c^{l}\right) \in \boldsymbol{\mu} \times \boldsymbol{\mu} \subseteq \Omega^{*}$. Now one can repeat the same argument using that $c^{2 i+1}$ is defined to conclude that $c^{2 i+2}$ is defined. Now that we know that $c^{2 i+2}$ is defined, we have, by induction, that $\left(c^{2}\right)^{i+1}=\left(c^{2}\right)^{i} \cdot c^{2}=c^{2 i} \cdot c^{2}=c^{2 i+2}$.

By the claim, we have that $a=\left(c^{2}\right)^{2^{\nu}}=c^{2^{\nu+1}}$. It remains to show that $\operatorname{ord}(c) \geq 2^{\nu+1}$. Let $i \in\left\{1, \ldots, 2^{\nu+1}\right\}$. Then if $i$ is even, we have $c^{i}=b^{\frac{i}{2}} \in \boldsymbol{\mu}$ by Lemma 6.11 since $\frac{i}{2} \leq 2^{\nu} \leq \operatorname{ord}(b)$. If $i$ is odd, then $c^{i}=c \cdot c^{\frac{i-1}{2}} \in \boldsymbol{\mu}$

for the same reason. Thus $c^{i} \in \boldsymbol{\mu}$ for all $i \in\left\{1, \ldots, 2^{\nu+1}\right\}$ and so $\operatorname{ord}(c) \geq$ $2^{\nu+1}$.

Theorem 6.13. $K$ is a neighborhood of 1 .

Proof. Fix $\nu>\mathbb{N}$. Then the internal set of all $\eta$ such that for each $x \in V_{\eta}$ there is $y \in \mathcal{U}^{*}$ such that $\operatorname{ord}(y) \geq 2^{\nu}$ and $x=y^{2^{\nu}}$ contains all infinite $\eta$. So by underspill, there is some $n>0$ in this set. Now given $x \in V_{n}$, there is $a \in \mathcal{U}^{*}$ such that $\operatorname{ord}(a) \geq 2^{\nu}$ and $x=a^{2^{\nu}}$. Let $\sigma:=2^{\nu}$. Then for $t \in I$, $X_{a}(t)=\operatorname{st}\left(a^{[t \sigma]}\right) \in \mathcal{U}$ and $X_{a}(1)=x$. Thus $\left[X_{a}\right] \in \mathcal{K}$ and $x=\left[X_{a}\right](1) \in K$. We have shown $V_{n} \subseteq K$ and this completes the proof.

\section{The Space $L(G)$}

Throughout this section, we assume that our (locally compact) $G$ is NSS. We fix a special neighborhood $\mathcal{U}$ of 1 in $G$. We will equip $L(G)$ with a topology in such a way that it becomes a locally compact finite dimensional real topological vector space, where the operations of scalar multiplication and vector addition are as previously defined. The section culminates with the important fact that locally compact NSS local groups are locally euclidean.

\section{The Compact-Open Topology}

We first recall that if $X, Y$ are topological spaces, we can turn $C(X, Y)$, the set of continuous maps from $X$ to $Y$, into a topological space by equipping it with the compact-open topology. This topology has as a subbasis the sets $B(V, U):=\{f \in C(X, Y) \mid f(V) \subseteq U\}$, where $V$ ranges over all compact subsets of $X$ and $U$ ranges over all open subsets of $Y$.

For $r \in \mathbb{R}^{>0}$, let $L_{r}(G):=\{X:(-r, r) \rightarrow G \mid X$ is a local 1-ps of $G\}$. Equip $C((-r, r), G)$ with the compact-open topology and $L_{r}(G)$ with the subspace 
topology. We will use the notation $B_{r}(V, U)$ to indicate a subbasic open set in $L_{r}(G)$. Now view

$$
L(G)=\underset{\lim }{\longrightarrow} L_{r}(G),
$$

where for $r \leq s, \rho_{s, r}: L_{s}(G) \rightarrow L_{r}(G)$ is the canonical restriction map. We also have the canonical maps $\rho_{r}: L_{r}(G) \rightarrow L(G)$ for each $r \in \mathbb{R}^{>0}$. Equip $L(G)$ with the final topology with respect to the maps $\rho_{r}$, that is $\mathcal{O} \subseteq L(G)$ is open if and only if $\rho_{r}^{-1}(\mathcal{O})$ is open in $L_{r}(G)$ for each $r \in \mathbb{R}^{>0}$.

In proving theorems about the topological space $L(G)$, the following facts are useful.

\section{Lemma 7.1.}

(1) For $r \leq s$, the map $\rho_{s, r}: L_{s}(G) \rightarrow \rho_{s, r}\left(L_{s}(G)\right)$ is a homeomorphism.

(2) The sets $D_{V, U}:=\{\mathbb{X} \in L(G) \mid V \subseteq \operatorname{domain}(\mathbb{X})$ and $\mathbb{X}(V) \subseteq U\}$ form a subbasis for the topology on $L(G)$, where $V$ ranges over all compact subsets of $\mathbb{R}$ and $U$ ranges over all open subsets of $G$.

Proof. It is clear that each $\rho_{s, r}$ is injective. To show that $\rho_{s, r}$ is continuous, it suffices to show that $\rho_{s, r}^{-1}\left(B_{r}(V, U)\right)$ is open in $L_{s}(G)$ for each compact $V \subseteq(-r, r)$ and open $U \subseteq G$. However, $\rho_{s, r}^{-1}\left(B_{r}(V, U)\right)=B_{s}(V, U)$, a subbasic open set in $L_{s}(G)$. Hence $\rho_{s, r}$ is continuous. We now must show that $\rho_{s, r}^{-1}: \rho_{s, r}\left(L_{s}(G)\right) \rightarrow L_{s}(G)$ is continuous. Let $B_{s}(V, U)$ be a subbasic open set in $L_{s}(G)$. Let $n$ be such that $\frac{1}{n} V \subseteq(-r, r)$. We already know that the partial map $p_{n}: G \rightarrow G$ given by $p_{n}(x)=x^{n}$ when $x^{n}$ is defined has an open domain and is continuous. Let $U^{\prime}=p_{n}^{-1}(U)$, an open subset of $G$. Then $\rho_{s, r}\left(B_{s}(V, U)\right)=B_{r}\left(\frac{1}{n} V, U^{\prime}\right)$, a subbasic open set in $L_{r}(G)$. We have thus proven (1).

We now show that each $D_{V, U}$ is open in $L(G)$. It is then obvious from the definition of the topology that they form a subbasis for the topology. Let $r \in \mathbb{R}^{>0}$. We must show $\rho_{r}^{-1}\left(D_{V, U}\right)$ is open in $L_{r}(G)$. If $V \subseteq(-r, r)$, then $\rho_{r}^{-1}\left(D_{V, U}\right)=B_{r}(V, U)$, a subbasic open set in $L_{r}(G)$. If $V \nsubseteq(-r, r)$, then

$$
\rho_{r}^{-1}\left(D_{V, U}\right)=\bigcup\left\{\rho_{s, r}\left(\rho_{s}^{-1}\left(D_{V, U}\right)\right) \mid V \subseteq(-s, s)\right\},
$$

which is open in $L_{r}(G)$ by (1).

We now describe the monad structure of $L(G)$ with respect to this topology.

Lemma 7.2. Let $\mathbb{X}, \mathbb{Y} \in L(G)^{*}$. Then

(1) $\mathbb{X} \in L(G)_{\mathrm{ns}}^{*}$ if and only if domain $(\mathbb{X}) \cap \mathbb{R} \neq \emptyset$ and for every $t \in \operatorname{domain}(\mathbb{X}) \cap \mathbb{R}, \mathbb{X}(t) \in G_{\mathrm{ns}}^{*}$ 
(2) If $\mathbb{X}, \mathbb{Y} \in L(G)_{\mathrm{ns}}^{*}$, then $\mathbb{X} \sim \mathbb{Y}$ if and only if domain $(\mathbb{X}) \cap \mathbb{R}=$ domain $(\mathbb{Y}) \cap \mathbb{R}$ and $\mathbb{X}(t) \sim \mathbb{Y}(t)$ for all $t \in \operatorname{domain}(\mathbb{X}) \cap \mathbb{R}$;

(3) If $\mathbb{X} \in L(G)_{\mathrm{ns}}^{*}$, then $\mathbb{X} \sim \mathbb{O}$ if and only if $\mathbb{R} \subseteq \operatorname{domain}(\mathbb{X})$ and for all $t \in \mathbb{R}, \mathbb{X}(t) \in \boldsymbol{\mu}$.

Proof. The forward direction of (1) is immediate from the definitions. To prove the converse, suppose there is $t \in \mathbb{R}^{>0}$ such that $t \in \operatorname{domain}(\mathbb{X})$ and $\mathbb{X}(t) \in G_{\mathrm{ns}}^{*}$ for every $t \in \operatorname{domain}(\mathbb{X}) \cap \mathbb{R}$. Define $\overline{\mathbb{X}} \in L(G)$ by $\overline{\mathbb{X}}(t)=\operatorname{st}(\mathbb{X}(t))$ for $t \in \operatorname{domain}(\mathbb{X}) \cap \mathbb{R}$. Then $\mathbb{X} \in \boldsymbol{\mu}(\overline{\mathbb{X}})$ and we have proven (1). Item (2) is immediate from the definitions and (3) is a special case of (2).

Lemma 7.3. $E: \mathcal{K} \rightarrow K$ is a homeomorphism.

Proof. We already know that $E$ is a bijection. Suppose $\mathbb{X} \in \mathcal{K}^{*}, \mathbb{X} \sim \mathbb{O}$. Then by the previous lemma, $E(\mathbb{X})=\mathbb{X}(1) \in \boldsymbol{\mu}$, so $E$ is continuous. Now suppose $\mathbb{X} \in \mathcal{K}^{*}$ with $E(\mathbb{X})=\mathbb{X}(1) \in \boldsymbol{\mu}$. We need to prove that $\mathbb{X} \sim \mathbb{O}$. Towards this end, suppose $y, z \in[0,1]^{*}$. If $y+z \in[0,1]^{*}$, then we already know that $\operatorname{st}(\mathbb{X}(y)) \cdot \operatorname{st}(\mathbb{X}(z))=\operatorname{st}(\mathbb{X}(y+z)) \in \mathcal{U}$. So suppose $y+z>1$. Then

$$
\begin{aligned}
\operatorname{st}(\mathbb{X}(y)) \cdot \operatorname{st}(\mathbb{X}(z)) & =\operatorname{st}(\mathbb{X}((y+z-1)+(1-z))) \cdot \operatorname{st}(\mathbb{X}(z)) \\
& =(\operatorname{st}(\mathbb{X}(y+z-1)) \cdot \operatorname{st}(\mathbb{X}((1-z))) \cdot \operatorname{st}(\mathbb{X}(z)) \\
& =\operatorname{st}(\mathbb{X}(y+z-1)) \cdot(\operatorname{st}(\mathbb{X}(1-z)) \cdot \operatorname{st}(\mathbb{X}(z))) \\
& =\operatorname{st}(\mathbb{X}(y+z-1)) \cdot \operatorname{st}(\mathbb{X}(1)) \\
& =\operatorname{st}(\mathbb{X}(y+z-1)) \in \mathcal{U} .
\end{aligned}
$$

This shows that $\left\{\operatorname{st}(\mathbb{X}(y)) \mid y \in I^{*}\right\}$ is a subgroup of $\mathcal{U}$, implying that $\mathbb{X}\left(I^{*}\right) \subseteq \boldsymbol{\mu}$. This implies that $\mathbb{R} \subseteq$ domain $(\mathbb{X})$ and $\mathbb{X}(\mathbb{R}) \subseteq \boldsymbol{\mu}$. By the last lemma, we have $\mathbb{X} \sim \mathbb{O}$.

Corollary 7.4. $\mathcal{K}$ is a compact neighborhood of $\mathbb{O}$ in $L(G)$.

Proof. That $\mathcal{K}$ is compact is immediate from Lemmas 4.8 and 7.3 , To see that $\mathcal{K}$ is a neighborhood of $\mathbb{O}$, choose an open $W \subseteq \mathcal{U}$ with $1 \in W$. Let $\mathcal{K}_{W}:=\{\mathbb{X} \in \mathcal{K} \mid \mathbb{X}(I) \subseteq W\}$. Clearly $\mathbb{O} \in \mathcal{K}_{W}$ and $\mathcal{K}_{W}$ is an open set in $L(G)$ by Lemma 7.1

Before we begin the proof of the next theorem, we will need to make one more remark about the topology on $L(G)$. Suppose $\mathbb{X} \in L(G)$ and $s \in \mathbb{R}^{>0}$ is such that $[-s, s] \subseteq \mathbb{X}^{-1}\left(\mathcal{U}_{2}\right)$. Let $U \subseteq \mathcal{U}_{2}$ be a neighborhood of 1 . Then the set

$$
N_{\mathbb{X}}(s, U):=\{\mathbb{Y} \in L(G) \mid \mathbb{Y}(t) \in \mathbb{X}(t) U \text { for all } t \in[-s, s]\}
$$


is a neighborhood of $\mathbb{X}$ in $L(G)$ and the collection

$$
\left\{N_{\mathbb{X}}(s, U) \mid U \text { is a neighborhood of } 1 \text { in } G\right\}
$$

forms a neighborhood base of $\mathbb{X}$ in $L(G)$. These facts follow immediately from the nonstandard characterization of the topology on $L(G)$.

Theorem 7.5. $L(G)$ is a locally compact, finite dimensional real topological vector space.

Proof. If we can prove that $L(G)$ is a topological vector space, then since it is locally compact by Corollary 7.4 , it is finite-dimensional by a theorem of Riesz. We first prove that scalar multiplication is continuous. Let $r \in \mathbb{R} \backslash\{0\}$ and let $f_{r}: L(G) \rightarrow L(G)$ be defined by $f_{r}(\mathbb{X})=r \cdot \mathbb{X}$. It suffices to show that $f_{r}^{-1}\left(D_{V, U}\right)$ is open in $L(G)$ for every subbasic open set $D_{V, U}$. However, it is easy to verify that $f_{r}^{-1}\left(D_{V, U}\right)=D_{r V, U}$, a subbasic open set in $L(G)$.

We next prove that + is continuous. We first show that + is continuous at $(\mathbb{O}, \mathbb{O})$. To do this, it suffices to show that for any subbasic open set $D_{V, U}$ of $L(G)$ containing $\mathbb{( O )}$ (so that $1 \in U$ ), there is a subbasic open set $D_{V, W}$ of $L(G)$ containing $\mathbb{O}$ such that $D_{V, W}+D_{V, W} \subseteq D_{V, U}$. Let $U_{1}$ be a compact neighborhood of 1 such that $U_{1} \subseteq U$. Let $Z \subseteq \boldsymbol{\mu}$ be an internally open set. Then by Lemma 6.2, we know that for every $a, b \in Z$ and for every $\sigma$ such that for all $i \in\{1, \ldots, \sigma\}, a^{i}$ and $b^{i}$ are defined and $a^{i}, b^{i} \in Z$, we have $(a b)^{\sigma}$ is defined and $(a b)^{\sigma} \in U_{1}^{*}$. Hence, by transfer, there is an open set $W$ such that for all $a, b \in W$ and all $n$ with $a^{i}$ and $b^{i}$ defined and $a^{i}, b^{i} \in W$ for all $i \in\{1, \ldots, n\}$, we have $(a b)^{n}$ defined and $(a b)^{n} \in U_{1}$. We claim that this is the desired $W$. Suppose $\mathbb{X}, \mathbb{Y} \in D_{V, W}$. Let $t \in V$ and suppose $\nu$ is such that $\frac{\nu}{\sigma} \sim t$. Then $(\mathbb{X}+\mathbb{Y})(t) \sim\left[\mathbb{X}\left(\frac{1}{\sigma}\right) \mathbb{Y}\left(\frac{1}{\sigma}\right)\right]^{\nu}$. Let $a:=\mathbb{X}\left(\frac{1}{\sigma}\right)$ and $b:=\mathbb{Y}\left(\frac{1}{\sigma}\right)$. By assumption, for all $i \in\{1, \ldots, \nu\}, a^{i}$ and $b^{i}$ are defined and $a^{i}, b^{i} \in W^{*}$. Thus, $(a b)^{\nu} \in U_{1}^{*}$, implying that $(\mathbb{X}+\mathbb{Y})(t) \in U_{1} \subseteq U$ and so $\mathbb{X}+\mathbb{Y} \in D_{V, U}$.

In order to finish the proof that + is continuous, it is enough to show that, for any $\mathbb{Y} \in L(G)$, the map $\mathbb{X} \mapsto \mathbb{X}+\mathbb{Y}: L(G) \rightarrow L(G)$ is continuous at $\mathbb{O}$ and the map $\mathbb{X} \mapsto \mathbb{X}-\mathbb{Y}: L(G) \rightarrow L(G)$ is continuous at $\mathbb{Y}$. Let $W \subseteq L(G)$ be a neighborhood of $\mathbb{Y}$ in $L(G)$. Fix $s \in \operatorname{domain}(\mathbb{Y})$ such that $\mathbb{Y}([-s, s]) \subseteq \mathcal{U}_{2}$. Fix a compact neighborhood $U$ of 1 in $G$ so that $N_{\mathbb{Y}}(s, U) \subseteq W$. Suppose $X \in \mu(\mathbb{O}) \subseteq L(G)^{*}$. By Lemma 6.2 , for all $i \leq[s \sigma]$ we have $\left(\mathbb{X}\left(\frac{1}{\sigma}\right) \mathbb{Y}\left(\frac{1}{\sigma}\right)\right)^{i}$ is defined and infinitely close to $\mathbb{Y}\left(\frac{i}{\sigma}\right)$. In particular, this means that for every $\mathbb{X} \in \mu(\mathbb{O})$, whenever $i \leq[s \sigma]$, we have $\left(\left(\mathbb{X}\left(\frac{1}{\sigma}\right) \mathbb{Y}\left(\frac{1}{\sigma}\right)\right)^{i}\right.$ is defined and

$$
\left(\mathbb{X}\left(\frac{1}{\sigma}\right) \mathbb{Y}\left(\frac{1}{\sigma}\right)\right)^{i} \in Y\left(\frac{i}{\sigma}\right) U^{*}
$$


By overspill, this gives a neighborhood $V$ of $\mathbb{O}$ in $L(G)$ such that for all $\mathbb{X} \in V$ we have $\left(\left(\mathbb{X}\left(\frac{1}{\sigma}\right) \mathbb{Y}\left(\frac{1}{\sigma}\right)\right)^{i}\right.$ is defined and

$$
\left(\mathbb{X}\left(\frac{1}{\sigma}\right) \mathbb{Y}\left(\frac{1}{\sigma}\right)\right)^{i} \in Y\left(\frac{i}{\sigma}\right) U^{*}
$$

whenever $i \leq[s \sigma]$. It now follows that for $\mathbb{X} \in V$, we have

$$
t \in \operatorname{domain}(\mathbb{X}+\mathbb{Y}) \text { and }(\mathbb{X}+\mathbb{Y})(t) \in \mathbb{Y}(t) U
$$

for all $t \in[-s, s]$, whence $\mathbb{X}+\mathbb{Y} \in N_{\mathbb{Y}}(s, U) \subseteq W$. This proves the continuity of $\mathbb{X} \mapsto \mathbb{X}+\mathbb{Y}$ at $\mathbb{O}$.

The continuity of $\mathbb{X} \mapsto \mathbb{X}-\mathbb{Y}$ at $\mathbb{Y}$ is proved in a similar fashion. Let $W \subseteq L(G)$ be a neighborhood of $\mathbb{O}$. Fix $s \in \operatorname{domain}(\mathbb{Y})$. Choose a compact neighborhood $U$ of 1 in $G$ so that $N_{\mathbb{O}}(s, U) \subseteq W$. Suppose $\mathbb{X} \in \mu(\mathbb{Y}) \subseteq$ $L(G)^{*}$. By Lemma 6.3, $\left(\mathbb{X}\left(\frac{1}{\sigma}\right) \mathbb{Y}\left(-\frac{1}{\sigma}\right)\right)^{i}$ is defined and infinitesimal whenever $i \leq[s \sigma]$. In particular,

$$
\left(\mathbb{X}\left(\frac{1}{\sigma}\right) \mathbb{Y}\left(-\frac{1}{\sigma}\right)\right)^{i} \in U^{*}
$$

whenever $i \leq[s \sigma]$. By overspill, this gives a neighborhood $V$ of $\mathbb{Y}$ in $L(G)$ such that for all $\mathbb{X} \in V$, we have $\left(\mathbb{X}\left(\frac{1}{\sigma}\right) \mathbb{Y}\left(-\frac{1}{\sigma}\right)\right)^{i}$ is defined and

$$
\left(\mathbb{X}\left(\frac{1}{\sigma}\right) \mathbb{Y}\left(-\frac{1}{\sigma}\right)\right)^{i} \in U^{*}
$$

whenever $i \leq[s \sigma]$. It now follows that for $\mathbb{X} \in V$, we have

$$
t \in \operatorname{domain}(\mathbb{X}-\mathbb{Y}) \text { and }(\mathbb{X}-\mathbb{Y})(t) \in U
$$

for all $t \in[-s, s]$, whence $\mathbb{X}-\mathbb{Y} \in N_{\mathbb{O}}(s, U) \subseteq W$.

We now need to prove that $L(G)$ is a vector space. There is only one vector space axiom which is not trivial to verify, namely that $r \cdot(\mathbb{X}+\mathbb{Y})=r \cdot \mathbb{X}+r \cdot \mathbb{Y}$. We first consider the case that $r \in \mathbb{Z}$. Let $a:=\mathbb{X}\left(\frac{1}{\sigma}\right)$ and $b:=\mathbb{Y}\left(\frac{1}{\sigma}\right)$. So $S(r \cdot \mathbb{X}+r \cdot \mathbb{Y})=\left(a^{r} b^{r}\right) G^{\mathrm{o}}(\sigma)$. Meanwhile, $S(r \cdot(\mathbb{X}+\mathbb{Y}))=\left((a b)^{r}\right) G^{\mathrm{o}}(\sigma)$. But $G(\sigma) / G^{\mathrm{o}}(\sigma)$ is abelian, so

$$
S(r \cdot(\mathbb{X}+\mathbb{Y}))=\left((a b)^{r}\right) G^{\mathrm{o}}(\sigma)=\left(a^{r} b^{r}\right) G^{\mathrm{o}}(\sigma)=S(r \cdot \mathbb{X}+r \cdot \mathbb{Y}) .
$$

We have thus proven this axiom for the case $r \in \mathbb{Z}$.

Once again suppose $r \in \mathbb{Z}$. We now know that $r \cdot\left(\frac{1}{r} \mathbb{X}+\frac{1}{r} Y\right)=\mathbb{X}+\mathbb{Y}$, i.e. that $\frac{1}{r} \cdot(\mathbb{X}+\mathbb{Y})=\frac{1}{r} \mathbb{X}+\frac{1}{r} \mathbb{Y}$. We thus know if $r \in \mathbb{Q}$, then $r \cdot(\mathbb{X}+\mathbb{Y})=r \cdot \mathbb{X}+r \cdot \mathbb{Y}$. We finally conclude that the axiom holds for all $r \in \mathbb{R}$ from the fact that the operations of addition and scalar multiplication are continuous.

Remark. Suppose $H$ and $H^{\prime}$ are locally compact pure local groups. Then the proof of Theorem 7.5 shows that $L(H)$ is a real topological vector space. The NSS assumption was only used to assert local compactness, and hence 
finite-dimensionality. Moreover, given any local morphism $f: H \rightarrow H^{\prime}$, one can verify that the map $L(f): L(H) \rightarrow L\left(H^{\prime}\right)$ is $\mathbb{R}$-linear and continuous.

Corollary 7.6. $G$ is locally euclidean.

Proof. Since $L(G)$ is a finite dimensional topological vector space over $\mathbb{R}$, we have an isomorphism $L(G) \cong \mathbb{R}^{n}$ of real vector spaces for some $n$, and any such isomorphism must be a homeomorphism. Hence, by Corollary [7.4, $\mathcal{K}$ contains an open neighborhood $U$ of 1 which is homeomorphic to an open subset of $\mathbb{R}^{n}$. Shrink $U$ if necessary so that $E(U) \subseteq W$, where $W$ is an open neighborhood of 1 in $G$ contained in $K$; such a $W$ exists by Theorem 6.13, Then by Lemma 7.3. $E(U)$ is an open neighborhood of 1 in $G$ homeomorphic to an open subset of $\mathbb{R}^{n}$.

\section{LOCAL H5 FOR NSS LOCAL GROUPS}

The goal of this section is to prove the Local H5 for NSS local groups using the local version of the Adjoint Representation Theorem.

In this section we assume $G$ is NSS and further assume that our special neighborhood has been chosen so that $\mathcal{U} \subseteq \mathcal{U}_{6}$.

\section{Local Adjoint Representation Theorem}

Fix $g \in \mathcal{U}_{6}$. Let $\mathbb{X} \in L(G)$ and $X \in \mathbb{X}$. Choose $r \in \operatorname{domain}(X) \cap \mathbb{R}^{>0}$ such that $X((-r, r)) \subseteq \mathcal{U}_{6}$. Define the function $g X g^{-1}:(-r, r) \rightarrow G$ by the rule $\left(g X g^{-1}\right)(t)=g X(t) g^{-1}$. We claim that $g X g^{-1}$ is a local 1-ps of $G$. Certainly $g X g^{-1}$ is continuous. Suppose $s, t, s+t \in(-r, r)$. Then since $g, g^{-1}, X(t), X(s) \in \mathcal{U}_{6}$, we have

$$
\begin{aligned}
\left(g X g^{-1}\right)(t+s) & =g(X(t) X(s)) g^{-1} \\
& =\left(g X(t) g^{-1}\right)\left(g X(s) g^{-1}\right) \\
& =\left(g X g^{-1}\right)(t) \cdot\left(g X g^{-1}\right)(s)
\end{aligned}
$$

Thus $g X g^{-1}$ is a local 1-ps of $G$ and $\operatorname{Ad}_{g}(\mathbb{X}):=g \mathbb{X} g^{-1}:=\left[g X g^{-1}\right] \in L(G)$ is easily seen to be independent of the choice of $X \in \mathbb{X}$ and $r \in \operatorname{domain}(X)$ as chosen above.

Lemma 8.1. Suppose $\sigma>\mathbb{N}, a \in G(\sigma)$ and $g \in \mathcal{U}_{6}$. Then 
(1) $\mathrm{gag}^{-1} \in G(\sigma)$;

(2) $\operatorname{Ad}_{g}\left(\left[X_{a}\right]\right)=\left[X_{g a g^{-1}}\right]$.

Proof. Let $\tau:=\operatorname{ord}(a)$. Note that $\sigma=\mathrm{O}(\tau)$.

Claim: If $i \in\{1, \ldots, \tau\}$, then $\left(g a g^{-1}\right)^{i}$ is defined and $\left(g a g^{-1}\right)^{i}=g a^{i} g^{-1}$.

We prove this by internal induction on $i$. This is certainly true for $i=1$ and we now assume that it holds for a given $i$ and that $i+1 \leq \tau$. Since we have assumed that $\left(g a g^{-1}\right)^{i}$ is defined, to show $\left(g a g^{-1}\right)^{i+1}$ is defined, it is enough to show, by Lemma 2.18, that $\left(\left(\mathrm{gag}^{-1}\right)^{k},\left(\mathrm{gag}^{-1}\right)^{l}\right) \in \Omega^{*}$ for all $k, l \in\{1, \ldots, i\}$ such that $k+l=i+1$. However, by induction, $\left(g a g^{-1}\right)^{k}=g a^{k} g^{-1}$ and $\left(g a g^{-1}\right)^{l}=g a^{l} g^{-1}$, and since $g, a^{k}, a^{l} \in \mathcal{U}_{6}^{*}$, we have $\left(g a^{k} g^{-1}, g a^{l} g^{-1}\right) \in \Omega^{*}$. We thus know that $\left(g a g^{-1}\right)^{i+1}$ is defined. Now $\left(g a g^{-1}\right)^{i+1}=\left(g a g^{-1}\right)^{i}\left(g a g^{-1}\right)=\left(g a^{i} g^{-1}\right)\left(g a g^{-1}\right)=g a^{i+1} g^{-1}$ since $g, a^{i}, a, g^{-1} \in \mathcal{U}_{6}^{*}$.

By the claim, if $i=\mathrm{o}(\sigma)$, then $\left(g a g^{-1}\right)^{i}$ is defined and $\left(g a g^{-1}\right)^{i}=g a^{i} g^{-1} \in \boldsymbol{\mu}$ since $a \in G(\sigma)$. This proves (1).

We now must prove (2). Fix $r \in \mathbb{R}^{>0}$ such that $[r \sigma] \leq \tau, X_{a}((-r, r)) \subseteq \mathcal{U}_{6}$ and $g X_{a}((-r, r)) g^{-1} \subseteq \mathcal{U}$. Then for $t \in(-r, r)$, we have

$$
\begin{aligned}
\left(\operatorname{Ad}_{g}\left(\left[X_{a}\right]\right)(t)\right) & =g X_{a}(t) g^{-1} \\
& =g\left(\operatorname{st}\left(a^{[t \sigma]}\right)\right) g^{-1} \\
& =\operatorname{st}\left(g a^{[t \sigma]} g^{-1}\right) \\
& =\operatorname{st}\left(\left(g a g^{-1}\right)^{[t \sigma]}\right) \\
& =X_{g a g^{-1}}(t),
\end{aligned}
$$

which finishes the proof.

The proof of the following lemma is routine.

Lemma 8.2. For $g \in \mathcal{U}_{6}, \operatorname{Ad}_{g}: L(G) \rightarrow L(G)$ is a vector space automorphism with inverse $\mathrm{Ad}_{g^{-1}}$.

Let $\operatorname{Aut}(L(G))$ denote the group of vector space automorphisms of $L(G)$. Suppose $\operatorname{dim}_{\mathbb{R}}(L(G))=n$. Take an $\mathbb{R}$-linear isomorphism $L(G) \cong \mathbb{R}^{n}$; it induces a group isomorphism $\operatorname{Aut}(L(G)) \cong \mathrm{GL}_{n}(\mathbb{R}) \subseteq \mathbb{R}^{n^{2}}$, and we take the 
topology on $\operatorname{Aut}(L(G))$ that makes this group isomorphism a homeomorphism. (This topology does not depend on the choice of $\mathbb{R}$-linear isomorphism $L(G) \cong \mathbb{R}^{n}$.) For $T \in \operatorname{Aut}(L(G))^{*}$, we see that $T$ is nearstandard if $T(\mathbb{X}) \in L(G)_{\mathrm{ns}}^{*}$ for all $\mathbb{X} \in L(G)$. For $T, T^{\prime} \in \operatorname{Aut}(L(G))_{\mathrm{ns}}^{*}$, we see that $T \sim T^{\prime}$ if and only if $T(\mathbb{X}) \sim T^{\prime}(\mathbb{X})$ for all $\mathbb{X} \in L(G)$.

Theorem 8.3. (Local Adjoint Representation Theorem) We have a morphism $\operatorname{Ad}: G \mid \mathcal{U}_{6} \rightarrow \operatorname{Aut}(L(G))$ of local groups given by $\operatorname{Ad}(g):=\operatorname{Ad}_{g}$.

Proof. Suppose $g, h, g h \in \mathcal{U}_{6}$ and $\left[X_{a}\right] \in L(G)$. Then

$$
\begin{aligned}
\operatorname{Ad}_{g h}\left(\left[X_{a}\right]\right) & =\left[X_{(g h) a(g h)^{-1}}\right] \\
& =\left[X_{\left.g\left(h a h^{-1}\right) g^{-1}\right]}\right. \\
& =\operatorname{Ad}_{g}\left(\operatorname{Ad}_{h}\left(\left[X_{a}\right]\right)\right) .
\end{aligned}
$$

Thus $\operatorname{Ad}(g h)=\operatorname{Ad}(g) \circ \operatorname{Ad}(h)$. All that remains to prove is that $\operatorname{Ad}$ is continuous. To do this, it suffices to prove that Ad is continuous at 1 . Suppose $a \in \boldsymbol{\mu}$ and $\mathbb{X} \in L(G)$.

Claim: $\operatorname{domain}(\mathbb{X})=\operatorname{domain}\left(\operatorname{Ad}_{a}(\mathbb{X})\right) \cap \mathbb{R}$.

In order to verify the claim, suppose $(-r, r) \subseteq$ domain $(\mathbb{X})$. We must verify that if $s, t, s+t \in(-r, r)$, then $\left(a \mathbb{X}(s) a^{-1}, a \mathbb{X}(t) a^{-1}\right) \in \Omega^{*}$ and $\left(a \mathbb{X}(s) a^{-1}\right)\left(a \mathbb{X}(t) a^{-1}\right)=a \mathbb{X}(s+t) a^{-1}$. From the fact that $(\mathbb{X}(s), \mathbb{X}(t)) \in \Omega$ and $a \in \boldsymbol{\mu}$, we have that $\left(a \mathbb{X}(s) a^{-1}, a \mathbb{X}(t) a^{-1}\right) \in \Omega^{*}$ and we have that $\left(a \mathbb{X}(s) a^{-1}\right)\left(a \mathbb{X}(t) a^{-1}\right)=a \mathbb{X}(s+t) a^{-1}$ from the usual calculations involving associativity when working with infinitesimals and nearstandard elements.

Now notice that if $t \in \operatorname{domain}(\mathbb{X})$, then $\left(\operatorname{Ad}_{a}(\mathbb{X})\right)(t)=a \mathbb{X}(t) a^{-1} \sim \mathbb{X}(t)$. So by Lemma 7.2. $\operatorname{Ad}_{a}(\mathbb{X}) \sim \mathbb{X}$. By the nonstandard characterization of the topology on $\operatorname{Aut}(L(G))$, we have $\operatorname{Ad}_{a} \sim \mathrm{id}_{L(G))}$, and hence Ad is continuous.

\section{Some Facts About Local Lie Groups}

In the global setting, the Adjoint Representation Theorem and some elementary Lie group theory imply that locally compact NSS groups are Lie groups. In the local group setting, we have to work a little harder. We need a few ways of obtaining local Lie groups and the results that follow serve this purpose.

Definition 8.4. A local group $G$ is abelian if there is a neighborhood $U$ of 1 in $G$ such that $U \subseteq \mathcal{U}_{2}$ and $a b=b a$ for all $a, b \in U$. 
Theorem 8.5. Suppose $G$ is abelian. Then $G$ is locally isomorphic to a Lie group.

Proof. Suppose we have chosen our special neighborhood $\mathcal{U}$ so small that elements of $\mathcal{U}$ commute with each other. Recall that we are still assuming that $\mathcal{U} \subseteq \mathcal{U}_{6}$.

Claim: Suppose $a, b \in \mathcal{U}$ are such that $(a b)^{n}, a^{n}$ and $b^{n}$ are all defined and $a^{i}, b^{i} \in \mathcal{U}$ for all $i \in\{1, \ldots, n\}$. Then $(a b)^{n}=a^{n} b^{n}$.

We prove the claim by induction on $n$. The case $n=1$ is trivial. Now suppose $(a b)^{n+1}$ is defined as are $a^{n+1}$ and $b^{n+1}$. Also suppose $a^{i}$ and $b^{i}$ are in $\mathcal{U}$ for all $i \in\{1, \ldots, n+1\}$. Then

$$
(a b)^{n+1}=(a b)^{n} \cdot(a b)=\left(a^{n} b^{n}\right) \cdot a b=a^{n} b^{n} a b=a^{n+1} b^{n+1} .
$$

This proves the claim.

Choose a symmetric open neighborhood $\mathcal{V}$ of $\mathbb{O}$ in $L(G)$ such that $\mathcal{V} \subseteq \mathcal{K}$ and $\mathbb{X}+\mathbb{Y} \in \mathcal{K}$ for all $\mathbb{X}, \mathbb{Y} \in \mathcal{V}$. By the transfer of the claim, if $\mathbb{X}, \mathbb{Y} \in \mathcal{V}$, then

$$
\begin{aligned}
(\mathbb{X}+\mathbb{Y})(1) & =\operatorname{st}\left(\left(\mathbb{X}\left(\frac{1}{\sigma}\right) \mathbb{Y}\left(\frac{1}{\sigma}\right)\right)^{\sigma}\right) \\
& =\operatorname{st}\left(\mathbb{X}\left(\frac{1}{\sigma}\right)^{\sigma} \mathbb{Y}\left(\frac{1}{\sigma}\right)^{\sigma}\right) \\
& =\mathbb{X}(1) \mathbb{Y}(1)
\end{aligned}
$$

After possibly shrinking $\mathcal{V}$, we can choose a symmetric open neighborhood $V$ of 1 in $G$ such that $E(\mathcal{V})=V$. This witnesses that the equivalence class of the local exponential map is a local isomorphism from $L(G)$ to $G$. Since $L(G)$ is a Lie group, we are done.

To prove the next lemma, we need the following well-known theorem.

Theorem 8.6. (von Neumann, [12], pg. 82) If $H$ is a hausdorff topological group which admits an injective continuous homomorphism into $\mathrm{GL}_{n}(\mathbb{R})$ for some $n$, then $H$ is a Lie group.

Lemma 8.7. Suppose $f: G \rightarrow \mathrm{GL}_{n}(\mathbb{R})$ is an injective morphism of local groups. Then $G$ is a local Lie group. 
Proof. Let $G^{\prime}=f(G) \subseteq \mathrm{GL}_{n}(\mathbb{R})$. Let $H$ be the subgroup of $\mathrm{GL}_{n}(\mathbb{R})$ generated by $G^{\prime}$. Let $\mathcal{F}^{\prime}$ be the filter of neighborhoods of 1 in $G$ and $\mathcal{F}^{\prime \prime}$ be the image of $\mathcal{F}^{\prime}$ under $f$. Finally, let $\mathcal{F}$ be the filter in $H$ generated by $\mathcal{F}^{\prime \prime}$. It is routine to verify that $\mathcal{F}$ satisfies the properties needed to make it a neighborhood filter at 1 for a topology on $H$ which makes $H$ a topological group. By the definition of this topology, the inclusion map $H \hookrightarrow \mathrm{GL}_{n}(\mathbb{R})$ is continuous. By von-Neumann's theorem, $H$ is a Lie group. The result now follows from the fact that $G$ is homeomorphic with $f(G)$ when $f(G)$ is given the induced topology from $H$.

We use the following theorem of Kuranishi [10] to finish our proof of the NSS case.

Theorem 8.8. Let $G$ be a locally compact local group and let $H$ be a normal sublocal group of $G$. Consider the local coset space $(G / H)_{W}$ as in Lemma 2.13. Suppose

(1) $H$ is an abelian local Lie group;

(2) $(G / H)_{W}$ is a local Lie group;

(3) there is a set $M \subseteq W$ containing 1 and $W^{\prime} \subseteq W$, an open neighborhood of 1 in $G$, such that for every $(z H) \cap W \in \pi\left(W^{\prime}\right)$, there is exactly one $a \in M$ such that $a \in(z H) \cap W$; moreover, the map $\pi\left(W^{\prime}\right) \rightarrow M$ that assigns to each element of $\pi\left(W^{\prime}\right)$ the corresponding a in $M$ is continuous.

Then $G \mid W$ is a local Lie group.

\section{Proof of the NSS Case}

We can now finish the proof that the Local H5 holds for NSS local groups. Let $G^{\prime}:=G \mid \mathcal{U}_{6}$. Recall the morphism of local groups Ad : $G^{\prime} \rightarrow \operatorname{Aut}(L(G))$. Then $H:=\operatorname{ker}(\mathrm{Ad})$ is a normal sublocal group of $G^{\prime}$. Since $H$ is also a locally compact NSS local group, we know that $H$ must have an open (in $H$ ) neighborhood $V$ of 1 ruled by local 1-parameter subgroups of $H$, which are also local 1-parameter subgroups of $G$. We claim $g h=h g$ for all $g, h \in V$, implying that $H$ is abelian. Note that if $\mathcal{U}^{\prime} \subseteq \mathcal{U}$ is a special neighborhood for $H$ and if $\mathbb{X} \in L(H)$ is such that $I \subseteq$ domain $(\mathbb{X})$ and $\mathbb{X}(I) \subseteq \mathcal{U}^{\prime}$, then $I \subseteq$ domain $\left(g \mathbb{X} g^{-1}\right)$. So for $g, h \in V$, writing $h=\mathbb{X}(1)$ for some $\mathbb{X} \in L(H)$, we have $g h g^{-1}=h$.

By Theorem 8.5, we have that a restriction of $H$ is a local Lie group. Let us abuse notation and denote this restriction, which is an equivalent sublocal group of $G^{\prime}$, by $H$. Note that if we let $G^{\prime \prime}:=\left(G^{\prime} / H\right)_{W}$ be some local coset space, then the adjoint representation induces an injective morphism $G^{\prime \prime} \rightarrow \operatorname{Aut}(L(G))$. Thus $G^{\prime \prime}$ is a local Lie group by Lemma 8.7. In order to 
finish the proof, we need to show that condition (3) of Kuranishi's theorem is satisfied.

Since $G^{\prime \prime}$ is a local Lie group, we can introduce canonical coordinates of the second kind. More precisely, we can find an open neighborhood $\mathcal{U}^{\prime}$ of 1 in $G^{\prime \prime}$ and a basis $\mathbb{X}_{1}^{\prime}, \ldots, \mathbb{X}_{r}^{\prime}$ of $L\left(G^{\prime \prime}\right)$ such that every element of $\mathcal{U}^{\prime}$ is of the form $\mathbb{X}_{1}^{\prime}\left(s_{1}\right) \cdots \mathbb{X}_{r}^{\prime}\left(s_{r}\right)$ for a unique tuple $\left(s_{1}, \ldots, s^{r}\right) \in\left[-\beta^{\prime}, \beta^{\prime}\right]^{r}$. Without loss of generality, we can suppose the closure of $\mathcal{U}^{\prime}$ is a special neighborhood of $G^{\prime \prime}$. Choose a special neighborhood $\mathcal{U}$ of $G^{\prime}$ such that $\pi(\mathcal{U}) \subseteq \mathcal{U}^{\prime}$. Let $Z \subseteq \mathcal{U}$ be an open neighborhood of 1 ruled by local 1-parameter subgroups of $G^{\prime}$. Fix $s_{0} \in\left(0, \beta^{\prime}\right)$ such that $\mathbb{X}_{1}^{\prime}\left(s_{0}\right), \ldots, \mathbb{X}_{r}^{\prime}\left(s_{0}\right) \in \pi(Z)$. Choose $x_{i} \in Z$ such that $\pi\left(x_{i}\right)=\mathbb{X}_{i}^{\prime}\left(s_{0}\right)$. Let $\mathbb{X}_{i} \in L\left(G^{\prime}\right)$ be such that $\mathbb{X}_{i}\left(s_{0}\right)=x_{i}$.

Let $\beta<s_{0}$ be so that $\mathbb{X}_{i}(s) \in \mathcal{U}_{2 r}$ if $|s| \leq \beta$ and $\mathbb{X}_{1}\left(s_{1}\right) \cdots \mathbb{X}_{r}\left(s_{r}\right) \in W$ if $\left|s_{i}\right| \leq \beta$ for all $i \in\{1, \ldots, r\}$. A uniqueness of root argument yields that $\pi\left(\mathbb{X}_{i}(s)\right)=\mathbb{X}_{i}^{\prime}(s)$ for $|s| \leq \beta$. Set

$$
M:=\left\{\mathbb{X}_{1}\left(s_{1}\right) \cdots \mathbb{X}_{r}\left(s_{r}\right)|| s_{i} \mid \leq \beta \text { for all } i=1, \ldots, r\right\}
$$

and let $W^{\prime} \subseteq W$ be an open neighborhood of 1 in $G$ contained in the image of the map

$$
\left(s_{1}, \ldots, s_{r}\right) \mapsto \mathbb{X}_{1}\left(s_{1}\right) \cdots \mathbb{X}_{r}\left(s_{r}\right):[-\beta, \beta]^{r} \rightarrow G .
$$

One can check that the choices of $M$ and $W^{\prime}$ fulfill condition (3) of Kuranishi's theorem.

\section{LOCALly EUClideAN LOCAL GROUPS ARE NSS}

In order to finish the proof of the Local H5, we must now prove that every locally euclidean local group is NSS. This proceeds in two stages: locally euclidean local groups are NSCS and locally connected NSCS local groups are NSS.

\section{Locally euclidean local groups are NSCS}

We will need the following facts.

Theorem 9.1. ([12], pg. 105) Every compact connected nontrivial hausdorff topological group has a nontrivial 1-parameter subgroup.

Lemma 9.2. Let $H$ be a topological group and $X: \mathbb{R} \rightarrow H$ a 1-parameter subgroup. 
(1) If $H_{1}$ is a closed subgroup of $H$, then either $X(\mathbb{R}) \subseteq H_{1}$ or there is a neighborhood $D$ of 0 in $\mathbb{R}$ such that $X(D) \cap H_{1}=\{1\}$.

(2) If $X$ is nontrivial, then there is a neighborhood $D$ of 0 in $\mathbb{R}$ on which $X$ is injective.

Proof. This is immediate from the well-known fact that a closed subgroup of the additive group of $\mathbb{R}$ different from $\{0\}$ and $\mathbb{R}$ is of the form $\mathbb{Z} r$ with $r \in \mathbb{R}^{>0}$.

Definition 9.3. Following Kaplansky [9], we call a topological space feebly finite-dimensional if, for some $n$, it does not contain a homeomorphic copy of $[0,1]^{n}$.

Clearly locally euclidean local groups are feebly finite-dimensional.

Lemma 9.4. If $G$ is feebly finite-dimensional, then $G$ is pure.

Proof. Assume $G$ is not pure, so we have a nondegenerate nonpure internal set $Q \subseteq \boldsymbol{\mu}$. Then by Lemma 3.9, the set $G_{U}:=\overline{G_{U}(Q)}$ is a nontrivial compact connected subgroup of $G$ contained in $U$, for every compact symmetric neighborhood $U$ of 1 in $G$ such that $U \subseteq \mathcal{U}_{2}$. We shall prove, by induction on $n$, that each of the aforementioned subgroups $G_{U}$ contains a homeomorphic image of $[0,1]^{n}$ for all $n$. The assertion is clearly true for $n=0$. Now assume it holds for a given $n$ and we will prove it holds for $n+1$. Let $U$ be a compact symmetric neighborhood of 1 in $G$ contained in $\mathcal{U}_{2}$. Theorem 9.1 yields a nontrivial 1-parameter subgroup $X: \mathbb{R} \rightarrow G_{U}$. Choose a compact symmetric neighborhood $W$ of 1 in $G$ such that $W \subseteq U$ and $X(\mathbb{R}) \nsubseteq W$. In particular, $X(\mathbb{R}) \nsubseteq G_{W}$. After replacing $X$ by $r X$ for suitable $r \in(0,1)$, we can assume $X$ is injective on $[0,1]$ and $X([0,1]) \cap G_{W}=\{1\}$.

By the inductive assumption, we have a map $Y:[0,1]^{n} \rightarrow G_{W}$ which is a homeomorphism onto its image. Since $G_{U}$ is a group and $G_{W} \subseteq G_{U}$, we can define the map $Z:[0,1] \times[0,1]^{n} \rightarrow G_{U}$ by $Z(t, s)=X(t) \cdot Y(s)$, which is clearly continuous. Suppose $Z(t, s)=Z\left(t^{\prime}, s^{\prime}\right)$ with $t \geq t^{\prime}$. Then we have $X\left(t-t^{\prime}\right)=Y\left(s^{\prime}\right) Y(s)^{-1} \in X([0,1]) \cap G_{W}=\{1\}$. Since $X$ is injective on $[0,1]$, we have $t=t^{\prime}$ and since $Y$ is injective, we have $s=s^{\prime}$. Hence $Z$ is injective and thus a homeomorphism onto its image.

The following lemma is the local group version of Theorem 5.1 in [6]. In the proof of Lemma 9.6 below, we will only use the global version of the result. However, in the proof of Theorem [9.9, we will truly use the weakening of the hypotheses in assuming that $G$ is only a local group. 
Lemma 9.5. Suppose $G$ is pure and $V$ is a neighborhood of 1 in $G$. Then $V$ contains a compact subgroup $H$ of $G$ and a neighborhood $W$ of 1 in $G$ such that every subgroup of $G$ contained in $W$ is contained in $H$.

Proof. Let $W$ be an internal neighborhood of 1 in $G^{*}$ such that $W \subseteq \boldsymbol{\mu}$. Note that if $E_{1}, \ldots, E_{\nu}$ is an internal sequence of internal subgroups of $G^{*}$ contained in $W$ and $a_{1}, \ldots, a_{\nu}$ is an internal sequence such that $a_{i} \in E_{i}$ for all $i \in\{1, \ldots, \nu\}$, then $a_{1} \cdots a_{\nu}$ is defined by Lemma 6.1. We let $S$ be the set of all products $a_{1} \cdots a_{\nu}$, where $E_{1}, \ldots, E_{\nu}$ is an internal sequence of internal subgroups of $G^{*}$ contained in $W$ and $a_{1}, \ldots, a_{\nu}$ is an internal sequence such that $a_{i} \in E_{i}$ for all $i \in\{1, \ldots, \nu\}$. By Lemma 6.1, $S$ is an internal subgroup of $G^{*}, S \subseteq \boldsymbol{\mu}$, and every internal subgroup of $G^{*}$ contained in $W$ is contained in $S$. Furthermore, if $H$ is the internal closure of $S$ in $G^{*}$, then $H$ is an internally compact internal subgroup of $G^{*}$ containing all of the subgroups of $W$ and $H \subseteq V^{*}$. The desired result follows by transfer.

Lemma 9.6. If $G$ is feebly finite-dimensional, then $G$ is NSCS.

Proof. Suppose that $G$ is feebly finite-dimensional but, towards a contradiction, that $G$ is not NSCS. We claim that for every compact symmetric neighborhood $U$ of 1 in $G$ contained in $\mathcal{U}_{2}$ and for every $n$, there is a compact subgroup of $G$ contained in $U$ which contains a homeomorphic copy of $[0,1]^{n}$. Assume this holds for a given $n$ and let $U$ be given. Since $G$ is pure, Lemma 9.5 implies that there is a compact symmetric neighborhood $V$ of 1 in $G$ containing 1 and a compact subgroup $H \subseteq U$ that contains every subgroup of $G$ contained in $V$. Since, by assumption, $V$ contains a nontrivial connected compact subgroup of $G$, we have a nontrivial 1-parameter subgroup $X$ of $H$. By shrinking $V$ if necessary, we can suppose $X(\mathbb{R}) \nsubseteq V$. By assumption, we have a compact subgroup $G(V) \subseteq V$ of $G$ and a homeomorphism $Y:[0,1]^{n} \rightarrow Y\left([0,1]^{n}\right) \subseteq G(V)$. After replacing $X$ by $r X$ for suitable $r \in(0,1)$, we can assume $X([0,1]) \subseteq V, X$ is injective on $[0,1]$, and $X([0,1]) \cap G(V)=\{1\}$. Since $H$ is a group, we can define $Z:[0,1] \times[0,1]^{n} \rightarrow H$ by $Z(s, t)=X(s) Y(t)$, which is clearly continuous. As in the proof of Lemma 9.4, one can check that $Z$ is injective, and thus a homeomorphism onto its image.

\section{Locally connected NSCS local groups are NSS}

The next lemma is the local analogue of Lemma 5.3 and Corollary 5.4 in [6]. The proof there has a gap, which can be filled by changing the hypotheses as in our lemma.

Lemma 9.7. Suppose $H$ is a normal sublocal group of $G$ which is totally disconnected. Let $\pi: G \rightarrow G / H$ be the canonical projection. Then $L(\pi)$ is surjective. 
Proof. Let $G^{\prime}:=(G / H)_{W}$, where $W$ is as in Lemma 2.13, and let $\mathbb{Y} \in L\left(G^{\prime}\right)$. We seek $\mathbb{X} \in L(G)$ so that $\pi \circ \mathbb{X}=\mathbb{Y}$. If $\mathbb{Y}$ is trivial, this is obvious. Thus we may assume, without loss of generality, that $1 \in \operatorname{domain}(\mathbb{Y})$ and $\mathbb{Y}(1) \neq 1_{G^{\prime}}$. Fix $\nu>\mathbb{N}$ and let $h:=\mathbb{Y}\left(\frac{1}{\nu}\right) \in \boldsymbol{\mu}\left(1_{G^{\prime}}\right)$. Take a compact symmetric neighborhood $V$ of $1_{G^{\prime}}$ in $G^{\prime}$ such that $\mathbb{Y}(1) \notin V$. Take a compact symmetric neighborhood $U$ of 1 in $G$ with $U \subseteq W$ such that $\pi(U) \subseteq V$. Since $\pi$ is an open map, we have $\boldsymbol{\mu}\left(1_{G^{\prime}}\right) \subseteq \pi(\boldsymbol{\mu})$. Choose $a \in \boldsymbol{\mu}$ with $\pi(a)=h$. Let $\sigma:=\operatorname{ord}_{U}(a)$. If $\nu \leq \sigma$, then $\pi\left(a^{\nu}\right) \in V^{*}$, contradicting the fact that $\pi\left(a^{\nu}\right)=h^{\nu}=1_{G^{\prime}} \notin V$. Thus we must have $\sigma<\nu$. If $i=\mathrm{o}(\sigma)$, then $\pi\left(\operatorname{st}\left(a^{i}\right)\right)=\operatorname{st}\left(h^{i}\right)=1_{G^{\prime}}$, so $G_{U}(a)=\left\{\operatorname{st}\left(a^{i}\right) \mid i=\mathrm{o}(\sigma)\right\}$ is a connected subgroup of $G$ contained in $H$. Since $H$ is totally disconnected, we must have $G_{U}(a)=\left\{1_{G}\right\}$, i.e. $a \in G(\sigma)$. Since $a \notin G^{\mathrm{o}}(\sigma)$, we have $\left[X_{a}\right] \neq \mathbb{O}$. Suppose $\sigma=\mathrm{o}(\nu)$ and let $t \in \operatorname{domain}\left(\pi \circ\left[X_{a}\right]\right)$. Then $\pi\left(\left[X_{a}\right](t)\right)=\operatorname{st}\left(h^{[t \sigma]}\right)=1$, whence $\left[X_{a}\right] \in L(H)$. Since $H$ is totally disconnected, $L(H)$ is trivial and hence $\left[X_{a}\right]=\mathbb{O}$, a contradiction. Thus we have $\sigma=(r+\epsilon) \nu$ for some $r \in \mathbb{R}^{>0}$ and infinitesimal $\epsilon \in \mathbb{R}^{*}$. Thus $\pi \circ\left[X_{a}\right]=r \mathbb{Y}$ and $\mathbb{X}:=\frac{1}{r}\left[X_{a}\right]$ is the desired lift of $\mathbb{Y}$.

We need one fact from the global setting that we include here for completeness. It is taken from [6].

Lemma 9.8. Suppose $G$ is a pure topological group such that there are no nontrivial 1-parameter subgroups $X: \mathbb{R} \rightarrow G$. Then $G$ has a neighborhood base at 1 of open subgroups of $G$. In particular, $G$ is totally disconnected.

Since locally euclidean local groups are locally connected, the next theorem, in combination with Lemma 9.6, finishes the proof of the Local H5.

Theorem 9.9. If $G$ is locally connected and NSCS, then $G$ is NSS.

Proof. Let $V$ be a compact symmetric neighborhood of 1 in $G$ such that $V$ contains no nontrivial connected subgroups. Choose an open neighborhood $W$ of 1 in $G$ with $W \subseteq V$ and a compact subgroup $H_{1}$ of $G$ with $H_{1} \subseteq V$ with the property that every subgroup of $G$ contained in $W$ is contained in $H_{1}$; these are guaranteed to exist by Lemma 9.5. Without loss of generality, we can assume $W \subseteq \mathcal{U}_{6}$. We now observe that if $a \in \boldsymbol{\mu}$ is degenerate, then $a \in H_{1}^{*}$, for the internal subgroup internally generated by $a$ is contained in $\boldsymbol{\mu} \subseteq W^{*}$. Since $G$ is pure, if $a \in \boldsymbol{\mu}$ is nondegenerate, then it is pure; for such an $a$, if $a \in H_{1}^{*}$, then $a^{\nu} \in H_{1}^{*} \subseteq V^{*}$ for all $\nu$, contradicting the fact that some power of $a$ lives outside of $V$. Hence, nondegenerate infinitesimals live outside of $H_{1}^{*}$.

Since $H_{1} \subseteq V, H_{1}$ admits no nontrivial 1-parameter subgroups, and hence $H_{1}$ must be totally disconnected by Lemma 9.8 . Choose an open (in $H_{1}$ ) 
subgroup $H$ of $H_{1}$ contained in $H_{1} \cap W$. Write $H=H_{1} \cap W_{1}$ with $W_{1}$ an open neighborhood of 1 in $G$ and $W_{1} \subseteq W$. Since $H$ is an open subgroup of $H_{1}$, it is also closed in $H_{1}$, and thus $H$ is a compact subset of $G$. This implies that the set $U:=\left\{a \in W_{1} \mid a H a^{-1} \subseteq W_{1}\right\}$ is open. Fix $a \in U$. Since $a H a^{-1}$ is a subgroup of $G$ contained in $W_{1} \subseteq W$, we have $a H a^{-1} \subseteq H_{1}$. Thus $a H a^{-1} \subseteq H_{1} \cap W_{1}=H$ implying that $H$, considered as a sublocal group of $G$, is normal. Replacing $U$ by $U \cap U^{-1}$, we can take $U$ as the associated normalizing neighborhood for $H$.

Note that $H \subseteq \mathcal{U}_{6}$ and $H^{6} \subseteq U$. Find symmetric open neighborhoods $W_{2}$ and $W_{3}$ of 1 in $G$ so that $H \subseteq W_{3} \subseteq \overline{W_{3}} \subseteq W_{2}$ and so that $W_{2} \subseteq \mathcal{U}_{6}$ and $W_{2}^{6} \subseteq U$; this is possible by the regularity of $G$ as a topological space and the fact that $H$ is compact. Let $G^{\prime}:=(G / H)_{W_{2}}$. Suppose $a \in \boldsymbol{\mu}$. If $a$ is degenerate, then we have that $a \in H_{1}^{*} \cap W_{1}^{*}=H^{*}$, whence $\pi(a)=1_{G^{\prime}}$. Now suppose that $a$ is nondegenerate. Let $\tau:=\operatorname{ord}_{\overline{W_{3}}}(a)$. Then $a^{\tau} \in\left(\overline{W_{3}}\right)^{*}$, but $a^{\tau+1} \in W_{2}^{*} \backslash\left(\overline{W_{3}}\right)^{*}$. Choose an open neighborhood $U^{\prime}$ of 1 in $G$ so that $U^{\prime} H \subseteq W_{3}$. Suppose $\pi\left(a^{\tau+1}\right)=\pi(x)$ for some $x \in\left(U^{\prime}\right)^{*}$. Then $a^{\tau+1}=x h$ for some $h \in H^{*}$, whence $a^{\tau+1} \in W_{3}^{*}$, a contradiction. Hence $\pi(a)^{\tau+1} \notin \pi\left(\left(U^{\prime}\right)^{*}\right)$. Meanwhile, for $i=\mathrm{o}(\tau)$, we have $\pi(a)^{i} \in \boldsymbol{\mu}\left(G^{\prime}\right)$. Thus $\pi(a)$ is pure in $G^{\prime}$ and we have shown that $G / H$ has no degenerate infinitesimals other than $1_{G^{\prime}}$; in other words, we have shown that $G^{\prime}$ is NSS.

Since $G$ and $G^{\prime}$ are pure, $L(\pi): L(G) \rightarrow L\left(G^{\prime}\right)$ is a continuous $\mathbb{R}$-linear map. Since $H$ is totally disconnected, $L(\pi)$ is surjective by Lemma 9.7. Note that if $\mathbb{X} \in \operatorname{ker}(L(\pi))$, then $\mathbb{X}$ can be considered a local 1-ps of $H$. Since $H$ is totally disconnected, $L(H)$ is trivial, and hence $\mathbb{X}=\mathbb{O}$. Since $L\left(G^{\prime}\right)$ is finite-dimensional, we can conclude that the map $L(\pi)$ is an isomorphism of real topological vector spaces.

Now take a special neighborhood $\mathcal{U}^{\prime}$ of $G^{\prime}$ and let $E^{\prime}: \mathcal{K}^{\prime} \rightarrow K^{\prime}$ denote the local exponential map for $G^{\prime}$. Take a connected neighborhood $\mathcal{U}$ of 1 in $G$ such that $\mathcal{U} \subseteq W_{2}$ and $\pi(\mathcal{U}) \subseteq K^{\prime}$. Let $x \in \mathcal{U}$. Since $E^{\prime}$ is a bijection, there is a unique $\mathbb{Y} \in \mathcal{K}^{\prime}$ such that $\pi(x)=E(\mathbb{Y})$. Since $L(\pi)$ is a bijection, there is a unique $\mathbb{X} \in L(G)$ such that $\pi \circ \mathbb{X}=\mathbb{Y}$. Thus we can write $x=\mathbb{X}(1) x(H)$ where $x(H) \in H$. One can easily verify that the map which assigns to each $x \in \mathcal{U}$ the above $\mathbb{Y} \in L\left(G^{\prime}\right)$ is continuous. From this and the fact that $L(\pi)$ is a homeomorphism, we see that the map $x \mapsto x(H): \mathcal{U} \rightarrow H$ is continuous. Since $\mathcal{U}$ is connected, $H$ is totally disconnected, and $1(H)=1$, it follows that $x(H)=1$ for all $x \in \mathcal{U}$. Now by the injectivity of $L(\pi)$ and $E^{\prime}$, we have that $\pi \mid \mathcal{U}$ is injective, implying that $G$ cannot have any subgroups other than $\{1\}$ contained in $\mathcal{U}$. We thus conclude that $G$ is NSS.

\section{REFERENCES}

[1] D. Brown and R.S. Houston, Cancellative semigroups on manifolds, Semigroup Forum 35 (1987), 279-302. 
[2] M. Davis, Applied Nonstandard Analysis, John Wiley and Sons Inc., 1977.

[3] L. van den Dries, Unpublished notes, 1981.

[4] A.M. Gleason, Groups without small subgroups, Ann. of Math. 56 (1952), 193-212.

[5] C.W. Henson, Foundations of Nonstandard Analysis: A Gentle Introduction to Nonstandard Extensions; Nonstandard Analysis: Theory and Applications, L. O. Arkeryd, N. J. Cutland, and C. W. Henson, eds., NATO Science Series C:, Springer, 2001.

[6] J. Hirschfeld, The nonstandard treatment of Hilbert's fifth problem, Transactions of the AMS, Vol. 321, No. 1. (Sep. 1990), 379-400.

[7] K.H. Hofmann and W. Weiss, More on cancellative semigroups on manifolds, Semigroup Forum 37 (1988), 93-111.

[8] R. Jacoby, Some theorems on the structure of locally compact local groups, Ann. Math. 66 (1957), 36-69.

[9] I. Kaplansky, Lie algebras and locally compact groups, University of Chicago Press, 1964.

[10] M. Kuranishi, On local euclidean groups satisfying certain conditions, Proc. Amer. Math. Soc. 1 (1950), 372-380.

[11] T. McGaffey, A partial solution to a generalization of Hilbert's local fifth problem: the standard part of a locally euclidean local nonstandard Lie group is an analytic Lie group.

[12] D. Montgomery and L. Zippin, Topological transformation groups, Interscience, New York, 1955.

[13] D. Montgomery and L. Zippin, Small subgroups of finite dimensional groups, Ann. of Math. 56 (1952), 213-241.

[14] P. Olver, Non-Associative local Lie groups, Journal of Lie Theory, Vol. 6 (1996), 23-51.

[15] C. Plaut, Associativity and the local version of Hilbert's fifth problem, Notes, University of Tennessee, 1993.

[16] L. Pontrjagin, Topological groups, Princeton University Press, 1939.

[17] M. Singer, One parameter subgroups and nonstandard analysis, Manuscripta Math. 18 (1976), 1-13.

University of Illinois, Department of Mathematics, 1409 W. Green street, URBANA, IL 61801

E-mail address: igoldbr2@math.uiuc.edu

$U R L$ : www.math.uiuc.edu/ ${ }^{\text {igoldbr2 }}$ 